\title{
Extending the alternating-offers protocol in the presence of competition: models and theoretical analysis
}

\author{
Nicola Gatti
}

Published online: 25 August 2009

(C) Springer Science + Business Media B.V. 2009

\begin{abstract}
The automation of bargaining is receiving a lot of attention in artificial intelligence research. Indeed, considering that bargaining is the most common form of economic transaction, its automation could lead software agents to reach more effective agreements. In the present paper we focus on the best-known bargaining protocol, i.e., the alternating-offers protocol. It provides an elegant mechanism whereby a buyer and a seller can bilaterally bargain. Although this protocol and its refinements have been studied extensively, no work up to the present provides an adequate model for bargaining in electronic markets. A result of these settings means that multiple buyers are in competition with each other for the purchase of a good from the same seller while, analogously, multiple sellers are in competition with each other for the sale of a good to the same buyer. The study of these settings is of paramount importance, as they will be commonplace in real-world applications. In the present paper we provide a model that extends the alternating-offers protocol to include competition among agents. ${ }^{1}$ Our game theoretical analysis shows that the proposed model is satisfactory: it effectively captures the competition among agents, equilibrium strategies are efficiently computable, and the equilibrium outcome is unique. The main results we achieve are the following. 1) With $m$ buyers and $n$ sellers and when the outside option (i.e., the possibility of leaving a negotiation to start a new one) is inhibited, we show that it can be reduced to a problem of matching and that can be addressed by using the Gale-Shapley's stable marriage algorithm. The equilibrium outcome is unique and can be computed in $O\left(l \cdot m \cdot n \cdot \bar{T}+(m+n)^{2}\right)$, where $l$ is the number of the issues and $\bar{T}$ is the maximum length of the bargaining. 2) With $m$ buyers and one seller and when the seller can exploit the outside option,
\end{abstract}

\footnotetext{
${ }^{1}$ The proposed model has been preliminarily presented in [19] and in [21].

N. Gatti $(\bowtie)$

Dipartimento di Elettronica e Informazione, Politecnico di Milano,

Piazza Leonardo da Vinci 32, 20133, Milano, Italy

e-mail:ngatti@elet.polimi.it
} 
we show that agents' equilibrium strategies can be computed in $O(l \cdot m \cdot \bar{T})$ and may be not unique. However, we show that a simple refinement of the agents' utility functions leads to equilibrium uniqueness.

Keywords Multiagent systems $\cdot$ Negotiations $\cdot$ Game theory

Mathematics Subject Classifications (2000) $91 \mathrm{~A} 10 \cdot 91 \mathrm{~A} 80$

\section{Introduction}

The automation of processes whereby electronic agents carry out economic transactions is one of the most challenging issues in artificial intelligence research [26]. The prominence of this challenge is widely acknowledged, as intelligent agents who negotiate with each other on behalf of human users are expected to lead to more efficient negotiations [42]. Although a vast literature studies this topic [7, 9, 11, 29, 31, 43], automated negotiation is still far from being widely employed in real-world electronic markets, since many problems remain open in the many existing negotiation settings.

Among the negotiation settings for commercial transactions, a crucial role is played by bilateral bargaining. A bilateral bargaining situation involves two parties who work in cooperation towards the creation of a commonly desirable surplus over whose distribution both parties are in conflict [44]. Bargaining situations are much used in real-world applications and constitute the most common of economic transactions. Moreover, the bargaining can be over one issue (e.g., price), in which case it is called single-issue bargaining, or it can be over multiple issues (e.g., price and quality), in which case it is called multi-issue bargaining. The formalized study of bargaining is commonly carried out by employing game-theoretical tools [34] with which one distinguishes the negotiation protocol and the negotiation strategies: the protocol sets the negotiation rules, specifying which actions are allowed and when [37]; a strategy is an agent's specific behavior in the negotiation. Given a bargaining protocol, the game-theoretical approach postulates that rational agents should employ strategies that are somehow in equilibrium [14]. Nash equilibrium constitutes the basic equilibrium concept.

The best known protocol for bilateral bargaining is the alternating-offers protocol. It was pioneered by Ståhl in [45], refined by Rubinstein in [38], and is considered the main protocol for bilateral negotiations [26]. The alternating-offers protocol has received a lot of attention, first in economic literature to analyze human transactions [36] and later in computer science to automate electronic transactions [29]. The alternating-offers protocol is simple: an agent makes an offer for the issue under dispute and the opponent can accept it, exit or make a counteroffer [9]; if the opponent accepts, the negotiation concludes with an agreement; if the opponent exits, the negotiation concludes with a disagreement; if a counteroffer is made, the process is repeated until the negotiation concludes with an agreement or a disagreement. Essentially, the alternating-offers bargaining protocol is an infinite extensive-form game, as both agents can make a real value proposal and no temporal deadline is present in the protocol. The payoffs of each agent depend on certain parameters: her reservation price, her time discount factor, and her temporal deadline. 
Although the analysis of the alternating-offers bargaining protocol in real-world settings is far from being completely understood, recently achieved computational results have been promising for its future employment. In [8] we see that the problem of efficiently negotiating multiple issues with complete information can be reduced in polynomial time (in the number of issues) to the problem of negotiating a single issue. This result was refined in [11], showing that this reduction can be accomplished in linear time (in the number of issues), since it can be formulated as a fractional knapsack problem [32]. Moreover, in [11] the authors show that the solution can be provided in polynomial time (in the number of agents' types) when the weights related to the issues in the agents' utility functions are uncertain. Finally, in $[6,20]$ the authors show that, in the presence of one-sided uncertain deadlines, the solution can be provided in time asymptotically independent of the number of types of the agent whose deadline is uncertain.

Up to now the literature has focused attention mainly on bargaining settings where there are several issues to negotiate and/or where there is uncertainty over one or more parameters in agents' utility functions. A crucial setting that currently does not find a satisfactory analysis in the literature is the setting in which the two bargainers act in a market of bargaining agents. The peculiarity of this setting is that multiple buyers are in competition with respect to the purchase of an item from the same seller while, analogously, multiple sellers are in competition with respect to the sale of an item to the same buyer. The analysis of such a setting is well known to be of paramount importance, indeed it would provide a natural approach to the understanding of how prices may emerge in markets as a direct consequence of the interaction of agents [44]. Moreover, it will be common in real-world applications. The original alternating-offers protocol does not provide a satisfactory model to describe the considered situation, since it exclusively captures purely bilateral settings [36]. The competition within markets of bargaining agents is elegantly captured in microeconomics/game theory by introducing agents' outside options in the negotiation mechanism $[1,25,40]$. The term "outside option" denotes the possibility for an agent of leaving the negotiation she is currently carrying out and negotiate with a different opponent [1]. Essentially, the introduction of the outside option within the alternating-offers protocol preserves the bilaterality of the negotiation (i.e., at each time point only two agents negotiate), but allows agents at each time point to change the opponent with whom they are negotiating. Obviously, at the equilibrium, the outside option will be exploited if it pays better than Rubinstein equilibrium, and ignored otherwise. The refinements of the alternatingoffers protocol proposed in microeconomics/game theory literature that deals with the outside option, e.g., see $[1,25,40]$, are based on assumptions too restrictive to be satisfactorily employed in real-world electronic transactions. To be more precise, they assume that all the buyers (sellers) have the same parameters, that the agents do not have deadlines, and that the process whereby the agents are matched is random, meaning the agents not being allowed to choose their opponent. Therefore, there is a gap between the bargaining models currently studied in literature and the bargaining situations commonly present in real-world electronic markets.

In the present paper we aim at closing this gap. More precisely, the original contributions we present in this paper are the following. (1) We provide a model that satisfactorily extends the alternating-offers protocol within an electronic market: (1.a) the model both rules the matching between agents and allows agents to exploit 
the outside option choosing explicitly the opponent with whom they want to start a new negotiation, (1.b) agents can have different parameters, have deadlines, and can negotiate over multiple issues, (1.c) equilibrium strategies are unique, and (1.d) agents' equilibrium strategies, in the presence of a unique buyer and a unique seller, collapse to the ones in the original alternating-offers model. (2) We game theoretically study the proposed model providing the following results. (2.a) When the outside option is inhibited, the problem can be reduced in polynomial time in the number of agents to one of matching and can be solved by using the GaleShapley's stable marriage algorithm [18]. The computational complexity of finding the equilibrium matching is $O\left(l \cdot m \cdot n \cdot \bar{T}+(m+n)^{2}\right)$ where $l$ is the number of issues, $m$ is the number of buyer agents, $n$ is the number of seller agents, and $\bar{T}$ is the longest deadline. (2.b) When there is a unique seller and the outside option is not inhibited (the reverse situation is analogous), agents' equilibrium strategies can be computed in time $O(l \cdot n \cdot \bar{T})$ and may not be unique. However, we show that a simple refinement of agents' utility functions allows for the equilibrium to be unique. We show also how the equilibrium strategies are affected by the presence of competition among the buyer agents. Finally, we discuss how the results presented in this paper can be employed to study settings with uncertainty.

The analysis of the settings with one-sided outside option and with complete information paves the way for further studies that we shall address in future. Initially, we remark the relevance of analyzing settings with one-sided outside option. We notice that a large number of concrete economic settings, although they present multiple buyers and sellers, can be reduced to one-sided competition situations. For instance, all the economic situations where there are $m$ buyer agents that desires an item $A$ and $n$ sellers, each one with $m$ or more units of $A$ to sell, can be reduced to $m$ independent economic situations each one with a specific buyer and $n$ sellers. That is, the competition involves only the sellers and not the buyers. This is common in markets with electronic shops, which can have a huge storage capacity. Furthermore, the analysis of settings with two-sided outside option will be the 'natural' extension of that with one-sided outside option. We remark the relevance of analyzing settings with complete information. This analysis is of paramount importance to derive properties inherent the negotiation mechanism, e.g., equilibrium uniqueness and unreasonable equilibrium strategies. Furthermore, this analysis is fundamental for studying the model with incomplete information. We recall that there not exists any algorithm that can be generally applied to solve bargaining with uncertainty. Extensions of the backward induction algorithm were proposed (e.g., see [11]), but these algorithms are not (generally) sound, as the authors showed in $[6,20]$. Algorithms for addressing bargaining with uncertainty are usually designed ad-hoc for the specific bargaining settings as extension of the algorithm for the computation of the strategies with complete information [20].

The paper is structured as follows. In Section 2 we introduce the model and the analysis of the classic alternating-offers bargaining protocol with complete information in the presence of agents' deadlines. In Section 3 we review the known published results on bargaining in markets and we propose our bargaining model. In Section 4 we game theoretically analyze the proposed model when the outside option is inhibited. In Section 5 we game theoretically analyze the settings where there is a unique seller and two buyers and the outside option is not inhibited. Section 6 briefly discusses how the proposed result can be employed to study settings with uncertainty. 
Section 7 concludes the paper. In Appendix A we extends the result discussed in Section 5 to the situation wherein there is a generic number of buyers. In Appendix B we report the proofs of the main theoretical results discussed along the paper.

\section{Bargaining with deadlines}

In this section we review the classic bilateral alternating-offers protocol where agents have deadlines, in order for us to introduce models, notations, and know results. We initially describe (Section 2.1) the bargaining protocol and subsequently we review (Section 2.2) how the equilibrium strategies can be produced in the presence of complete information.

\subsection{Bargaining model}

The alternating-offers bargaining protocol is an extensive-form game where two agents, a buyer $\mathbf{b}$ and a seller $\mathbf{s}$, try to agree on the value of one or more issues. For the sake of presentation, in the present paper we study single-issue settings and we discuss how the results can be extended to multi-issue settings. This is because, as is shown in $[8,11]$, with complete information the study of multi-issue settings can be reduced (in linear time) to the study of single-issue settings. We discuss how this reduction can be addressed in the next section. The alternating-offers protocol is characterized by a player function $\iota: \mathbb{N} \rightarrow\{\mathbf{b}, \mathbf{s}\}$ that returns the agent which acts at time $t$ and is such that $\iota(t) \neq \iota(t+1)$. The agent $\iota(0)$ that opens the negotiation is a parameter of the protocol. At time $t>0$ agent $\iota(t)$ can make one of the three following actions:

- $\quad$ (i) offer $(x)$, where $x \in \mathbb{R}$,

- (ii) exit,

- (iii) accept.

At $t=0$ agent $\iota(0)$ can choose only $(i)$ or $(i i)$. If $\sigma_{\iota(t)}(t)=$ accept the game stops and the outcome is the agreement $(x, t)$, where $x$ is the value such that $\sigma_{\iota(t-1)}(t-1)=$ offer $(x)$. This is to say that the agents have agreed on the value $x$ at $t$. If $\sigma_{\iota(t)}(t)=$ exit the game stops and the outcome is NoAgreement. This is to say that the agents have not found an agreement. Otherwise the bargaining continues to the next time point.

The utility of an agreement $(x, t)$ for agent $i$ is given by a utility function $U_{i}$ : $(\mathbb{R} \times \mathbb{N}) \cup\{$ No Agreement $\} \rightarrow \mathbb{R}$ that depends on three parameters of agent $i$ : the reservation price $R P_{i} \in \mathbb{R}^{+}$, the temporal discount factor $\delta_{i} \in(0,1]$, and the deadline $T_{i} \in \mathbb{N}, T_{i}>0$. The values $R P_{\mathbf{b}}, R P_{\mathbf{s}}, \delta_{\mathbf{b}}, \delta_{\mathbf{s}}, T_{\mathbf{b}}$, and $T_{\mathbf{s}}$ are parameters of the protocol. If the outcome of the bargaining is an agreement $(x, t)$, then the utility functions $U_{\mathbf{b}}$ and $U_{\mathbf{s}}$ are respectively:

$$
U_{\mathbf{b}}(x, t)=\left\{\begin{array}{ll}
\left(R P_{\mathbf{b}}-x\right) \cdot \delta_{\mathbf{b}}^{t} & \text { if } t \leq T_{\mathbf{b}} \\
-1 & \text { otherwise }
\end{array}, \quad U_{\mathbf{s}}(x, t)=\left\{\begin{array}{ll}
\left(x-R P_{\mathbf{s}}\right) \cdot \delta_{\mathbf{s}}^{t} & \text { if } t \leq T_{\mathbf{s}} \\
-1 & \text { otherwise }
\end{array} .\right.\right.
$$

If the outcome is NoAgreement, then agents do not receive any gain from the negotiation, namely, $U_{\mathbf{b}}($ NoAgreement $)=U_{\mathbf{s}}($ NoAgreement $)=0$. Notice that the assignment of a strictly negative value (we have chosen by convention the value -1 ) 
to $U_{i}$ after agent $i$ 's deadline allows one to capture the essence of the deadline: an agent, after her deadline, strictly prefers to exit the negotiation rather than to reach any agreement.

Finally, we standardly assume the feasibility of the problem, i.e., $R P_{\mathbf{b}} \geq R P_{\mathbf{s}}$, and the rationality of the agents, i.e., it is common knowledge that each agent will act to maximize her utility.

\subsection{Equilibrium strategies}

When the information is complete the appropriate solution concept for the game we are dealing with is the subgame perfect equilibrium [24]. Subgame perfect equilibrium prescribes strategies that are of equilibrium in every possible subgame. These strategies can easily be found by employing the backward induction method [13]. We briefly review the use of backward induction in this setting since it will play a crucial role in the analysis of the extension we propose in Section 3.2; details can be found in [34].

The time point $\bar{T}$ where the game rationally stops is initially determined: it is $\bar{T}=\min \left\{T_{\mathbf{b}}, T_{\mathbf{s}}\right\}$. The equilibrium outcome of every subgame starting from $t \geq \bar{T}$ is No Agreement, since at least one agent, gaining negative utility from reaching any agreement beyond $\bar{T}$, will exit. Therefore, at $\bar{T}$ agent $\iota(\bar{T})$ would accept any offer $x$ which gives her a utility no worse than NoAgreement, namely, any offer $x$ such that $U_{\iota(\bar{T})}(x, \bar{T}) \geq 0$. That is, any $x \leq R P_{\mathbf{b}}$ if $\iota(\bar{T})=\mathbf{b}$ and any $x \geq R P_{\mathbf{s}}$ if $\iota(\bar{T})=\mathbf{s}$. From $t=\bar{T}-1$ back to $t=0$, it is possible to find the optimal offer agent $\iota(t)$ can make at $t$, if she makes an offer, and the offers that she would accept. We denote by $x^{*}(t)$ the optimal offer of agent $\iota(t)$ at $t$. The optimal offer an agent can make is the offer that, among all the ones that her opponent will accept at the next time point, gives her the maximum utility. More precisely, $x^{*}(t)$ is the offer such that at $t+1$, if $t<\bar{T}-1$, agent $\iota(t+1)$ is indifferent between accepting it and rejecting it to make her optimal offer $x^{*}(t+1)$ and, if $t=\bar{T}-1$, agent $\iota(t+1)$ is indifferent between accepting it and choosing exit. On the equilibrium path, although at $t$ agent $\iota(t)$ is indifferent between accepting $x^{*}(t-1)$ and rejecting it to make her optimal action between $\operatorname{offer}\left(x^{*}(t)\right)$ and exit, agent $\iota(t)$ will always accept $x^{*}(t-1)$. It can be shown indeed that there is no equilibrium when agent $\iota(t)$ rejects $x^{*}(t-1)$. Essentially, the non-existence of the equilibrium is due to the fact that $x$ is a real number: if $\mathbf{b}$ (or $\mathbf{s}$ ) rejects the offer $x^{*}(t-1)$ at $t$, then the optimal offer of $\mathbf{s}$ (or $\left.\mathbf{b}\right)$ at $t-1$ does not exist, being $x^{*}(t-1)-\epsilon\left(\right.$ or $\left.x^{*}(t-1)+\epsilon\right)$ with $\epsilon>0$ and $\epsilon \rightarrow 0$; details on the proof can be found in [34]. In formula, $x^{*}(t)$ is such that $U_{l(t+1)}\left(x^{*}(t), t\right)=U_{l(t+1)}\left(x^{*}(t+1), t+1\right)$ if $t<\bar{T}-1$ and $U_{\iota(t+1)}\left(x^{*}(t), t\right)=0$ if $t=\bar{T}-1$. The offers agent $\iota(t)$ would accept at $t$ are all those offers that give her a utility no worse than the utility given by offering $x^{*}(t)$. That is, any $x \leq x^{*}(t)$ if $\iota(t)=\mathbf{b}$ and any $x \geq x^{*}(t)$ if $\iota(t)=\mathbf{s}$. In summary, the equilibrium strategies in any subgame starting from $0 \leq t<\bar{T}$ prescribe that agent $\iota(t)$ offers $x^{*}(t)$ at $t$ and agent $\iota(t+1)$ accepts it at $t+1$.

In order to provide a recursive formula for $x^{*}(t)$, we introduce the notion of backward propagation [7]: given value $x$ and agent $i$, we call backward propagation of value $x$ for agent $i$ the value $y$ such that $U_{i}(y, t-1)=U_{i}(x, t)$; we shall employ the arrow notation $x_{\leftarrow i}$ for backward propagations. In formula, $x_{\leftarrow \mathbf{b}}=R P_{\mathbf{b}}-\left(R P_{\mathbf{b}}-\right.$ $x) \cdot \delta_{\mathbf{b}}$ and $x_{\leftarrow \mathbf{s}}=R P_{\mathbf{s}}+\left(x-R P_{\mathbf{s}}\right) \cdot \delta_{\mathbf{s}}$. If a value $x$ is propagated backward $n$ times for agent $i$, we write $x_{\leftarrow n[i]}$; for instance $x_{\leftarrow 2[i]}=\left(x_{\leftarrow i}\right)_{\leftarrow i}$. If a value is propagated 
backward for more than one agent according to a given sequence, we list them left to right in the subscript; for instance $x_{\leftarrow i 2[j]}=\left(\left(x_{\leftarrow i}\right)_{\leftarrow j}\right)_{\leftarrow j}$ and $x_{\leftarrow i j i}=\left(\left(x_{\leftarrow i}\right)_{\leftarrow j}\right)_{\leftarrow i}$. (Notice that the definition of backward propagation is general and does not depend on the specific game we are studying.) The values of $x^{*}(t)$ can be calculated from $t=\bar{T}-1$ back to $t=0$ as follows:

$$
x^{*}(t)=\left\{\begin{array}{ll}
R P_{\iota(t+1)} & \text { if } t=\bar{T}-1 \\
\left(x^{*}(t+1)\right)_{\leftarrow \iota(t+1)} & \text { if } t<\bar{T}-1
\end{array} .\right.
$$

Finally, the equilibrium strategies can be easily defined on the basis of $x^{*}(t)$ as collections of choice rules:

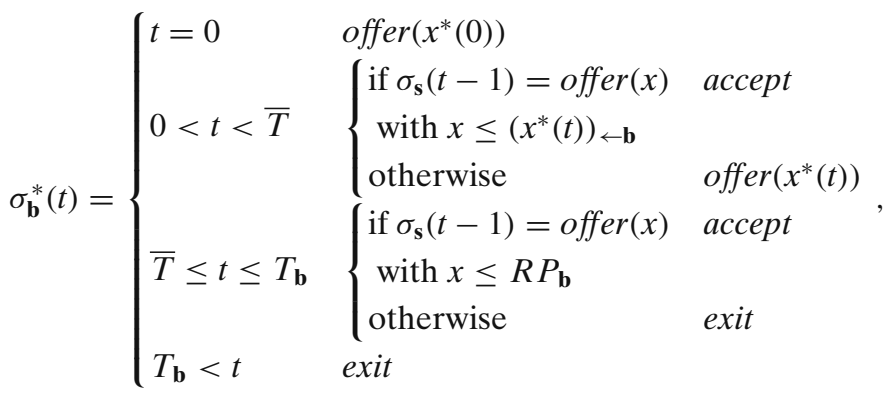

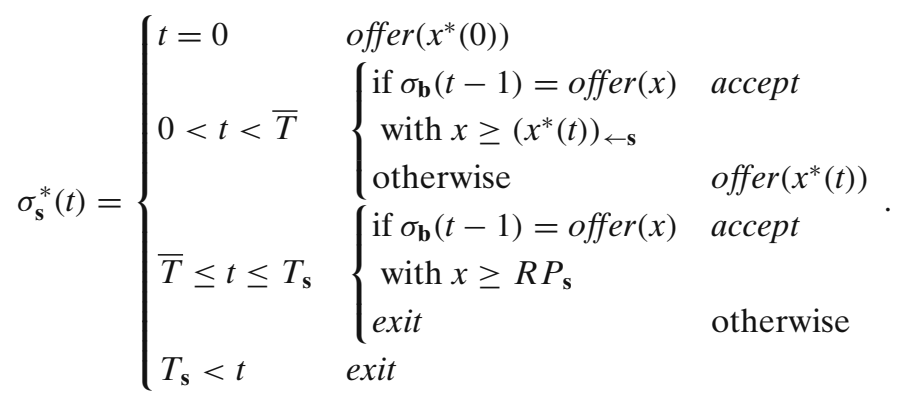

Now we state the following proposition, which is a well known published result; the proof can be found in [34].

Proposition 2.1 The above strategies constitute the unique subgame perfect equilibrium of the game described in Section 2.1.

We briefly review the proof, since it will be useful in the analysis we make in Section 5. We recall that an extensive-form game admits multiple equilibria when agents, at some decision nodes, have multiple optimal actions. It can be easily observed that at each step of the above backward induction construction all the actions of the agents are dominated but two: to accept $x^{*}(t)$ and to reject it to make the optimal action between $\operatorname{offer}\left(x^{*}(t+1)\right)$ and exit. Therefore, any possible equilibrium strategy cannot prescribe actions different from these. We have previously shown that there cannot be any equilibrium strategy that prescribes agent $\iota(t)$ to reject $x^{*}(t-1)$. Therefore, although at each time point agents have actions that are indifferent to their optimal actions, the equilibrium strategies are unique. 
Finally, it can be observed that the above equilibrium strategies can be found in time linear in $\bar{T}$, namely, $O(\bar{T})$.

In Fig. 1 and in Fig. 2 we depict two examples of backward induction constructions. The parameters in both examples are $R P_{\mathbf{b}}=1, \delta_{\mathbf{b}}=.95, T_{\mathbf{b}}=11, R P_{\mathbf{s}}=0, \delta_{\mathbf{s}}=.95$, and $T_{\mathbf{s}}=12$. The situation depicted in Fig. 1 is characterized by $\iota(0)=\mathbf{s}$, whereas in the situation depicted in Fig. $2 \iota(0)=\mathbf{b}$. We report at each time $t$ point the optimal offer of agent $\iota(t)$. The lines connecting two consecutive offers are isoutility curves. In both examples $\bar{T}=\min \left\{T_{\mathbf{b}}, T_{\mathbf{s}}\right\}=11$. Consider the game depicted in Fig. 1 . The agent that acts at $t=11$ is $\mathbf{b}$. Therefore, $x^{*}(10)=R P_{\mathbf{b}}=1, x^{*}(9)=\left(x^{*}(10)\right)_{\leftarrow \mathbf{s}}=.95$, $x^{*}(8)=\left(x^{*}(9)\right)_{\leftarrow \mathbf{b}}=.9525$, and so on. Consider the game depicted in Fig. 2 . The agent that acts at $t=11$ is $\mathbf{s}$. Therefore, $x^{*}(10)=R P_{\mathbf{s}}=0, x^{*}(9)=\left(x^{*}(10)\right)_{\leftarrow \mathbf{b}}=.05$, $x^{*}(8)=\left(x^{*}(9)\right)_{\leftarrow \mathbf{s}}=.0475$, and so on. It is worth noting that in the alternatingoffers bargaining protocol the agent which acts at $t=\bar{T}-1$ gains benefit from the protocol independently of her deadline, whereas the agent with the latest deadline cannot exercise her strength. Consider the game depicted in Fig. 1: s must accept her reservation price at $t=10$ even when her deadline is much longer than the opponent's one. This drawback of the protocol is partially mitigated in the presence of competition among several buyers. As we shall show in Section 5, in the presence of several possible opponents the position of $\mathbf{s}$ gets stronger: $\mathbf{s}$ could prefer to leave the current negotiation and start a new one instead of accepting her reservation price.

We briefly review how multiple issue bargaining settings can be studied; details can be found in $[7,8]$. In presence of multiple issues, several procedures can be employed to negotiate the issues, e.g., sequentially or simultaneously. The most efficient procedure (in terms of Pareto efficiency) is the in-bundle procedure [11], i.e., when agents simultaneously negotiate all the issues, and we therefore limit our analysis to this procedure. Agents' equilibrium strategies are very similar to the ones in the presence of a single issue: they can be defined on a sequence of optimal offers. These offers are $l$-tuples, where $l$ is the number of the issues, and specify one value for each issue, e.g., $\mathbf{x}^{*}(t)=\left\langle x_{1}, \ldots, x_{l}\right\rangle$. As is with single-issue bargaining, agents will accept any offer that is not worse than offering their optimal offer and at the

Fig. 1 Backward induction constructions with $R P_{\mathbf{b}}=1$, $\delta_{\mathbf{b}}=.95, T_{\mathbf{b}}=11, R P_{\mathbf{s}}=0$, $\delta_{\mathbf{s}}=.95, T_{\mathbf{s}}=12$, and $\iota(0)=\mathbf{s}$

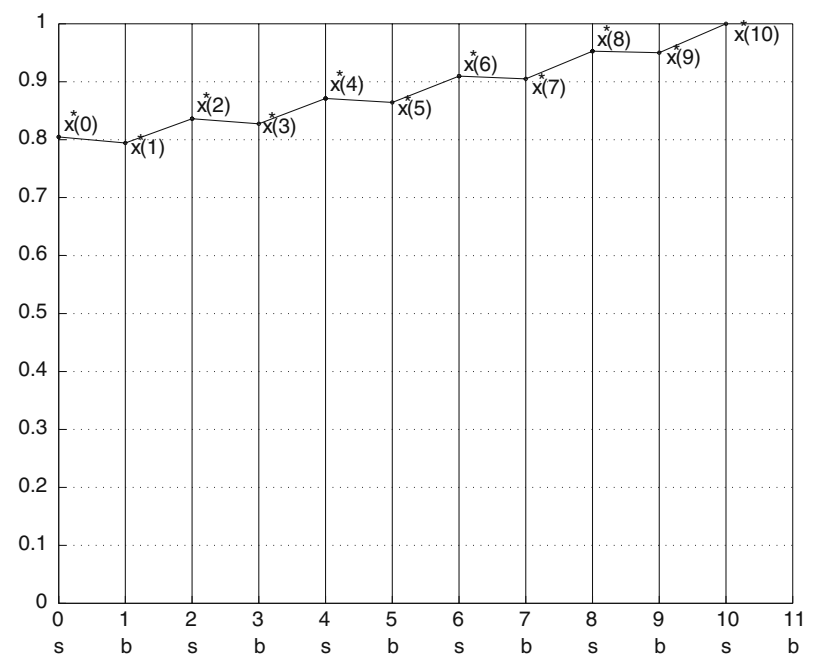


Fig. 2 Backward induction constructions with $R P_{\mathbf{b}}=1$, $\delta_{\mathbf{b}}=.95, T_{\mathbf{b}}=11, R P_{\mathbf{s}}=0$, $\delta_{\mathbf{s}}=.95, T_{\mathbf{s}}=12$, and $\iota(0)=\mathbf{b}$

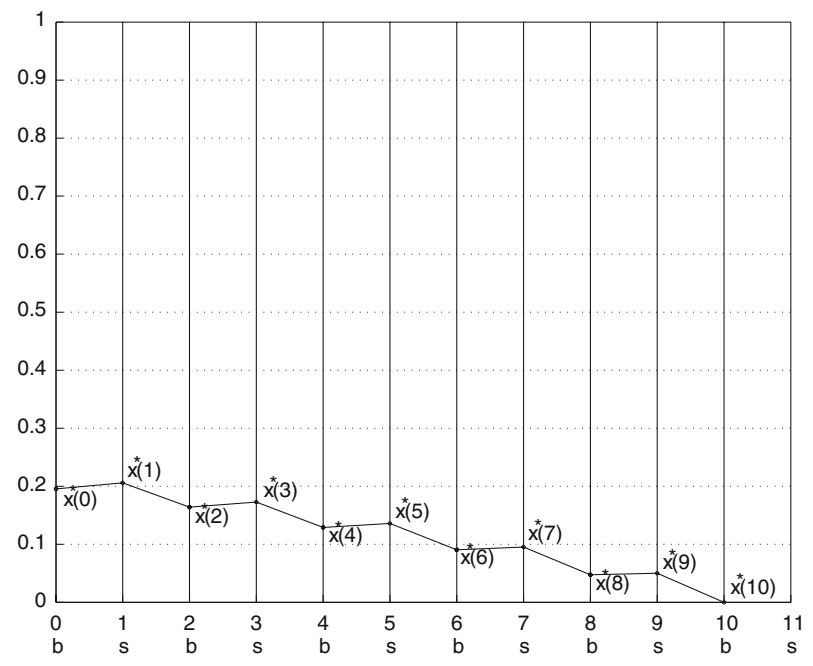

equilibrium each optimal offer $\mathbf{x}^{*}(t)$ will be accepted at $t+1$. The sequence of the optimal offers $\mathbf{x}^{*}(t)$ s can be found by backward induction, but an extension of the backward propagation of an offer we defined above is needed. More precisely, the backward induction construction is the same: at each time point $t$ the optimal offer $\mathbf{x}^{*}(t)$ of agent $\iota(t)$ is the offer such that agent $\iota(t+1)$ is indifferent at $t+1$ between accepting it and making her optimal offer $\mathbf{x}^{*}(t+1)$. In formula, $U_{l(t+1)}\left(\mathbf{x}^{*}(t), t+1\right)=$ $U_{l(t+1)}\left(\mathbf{x}^{*}(t+1), t+2\right)$. The difference between the multiple issue situation and the single issue situation depends on how $\mathbf{x}^{*}(t)$ can be computed given $\mathbf{x}^{*}(t+1)$. With a single issue, the backward propagation $y=x_{\leftarrow i}$ is a function that maps an offer $x \in \mathbb{R}$ at $t$ to an offer $y \in \mathbb{R}$ at $t-1$ (keeping constant the utility of agent $i$ ) and the calculation of $y$ can be easily accomplished in closed form. With multiple issues, the backward propagation $\mathbf{y}=\mathbf{x}_{\leftarrow i}$ maps an offer $\mathbf{x} \in \mathbb{R}^{l}$ at $t$ to an offer $\mathbf{y} \in \mathbb{R}^{l}$ at $t-1$ (keeping constant the utility of agent $i$ ) and the calculation of $\mathbf{y}$ requires the solution of a convex programming problem [7]. In the most common situations where the utility functions are linear, this problem can be solved in linear time in the number of issues, being a fractional knapsack problem (a proof is provided in [10]).

\section{Bargaining in markets}

In this section we review (Section 3.1) the main contributions presented in the literature on the analysis of bargaining within markets and we present (Section 3.2) a bargaining model that extends the model described in Section 2.1.

\subsection{Bargaining and markets in literature}

As underlined in [36] and in [44] bargaining theory provides a natural approach to the understanding of how prices may emerge in markets as a consequence of the direct interaction of agents. For the sake of argument, consider a market populated by one seller and multiple buyers. The buyers are 'naturally' in competition, since every 
buyer would receive a gain from drawing up a contract with the seller rather than abstaining from doing so, but only one buyer will make it. Therefore, we expect that the position of the seller agent gets stronger and that her gain increases with respect to the situation in which there is no competition among buyers. Obviously, the larger the number of the buyers, the stronger the position of the seller and the higher the prices of the contract which the seller can draw up. The original alternating-offers protocol presented in Section 2.1 does not provide a satisfactory description of the situation where agents act within a market, not taking into account explicitly the competition among the agents. This inadequacy of the original alternating-offers protocol has pushed researchers to extend this model. In particular, the two main issues to address when taking into account agents' competition within a market are:

1. static competition: an agent that is not negotiating can choose the opponent with whom to negotiate among a number of agents, e.g., given a seller and multiple buyers, the seller will choose to negotiate with the buyer from whom she expects to gain the maximum utility;

2. dynamic competition: an agent that is negotiating can choose to leave the negotiation she is carrying out to start a new negotiation with a different opponent. ${ }^{2}$

Technically speaking, addressing the first point essentially requires complementing the original protocol by introducing the rules for the matching of the agents. It can be easily observed that these rules do not alter the negotiation process: once two agents are matched, they negotiate as prescribed in the previous section. Addressing the second point instead leads to a different negotiation protocol, thus producing different agreements between the agents. We briefly review the main related works.

A large amount of works on dynamic competition in bargaining can be found in classical microeconomics/game theory literature [44]. They can be grouped in two main areas according to the perspective of their analysis: they can analyze markets in the small or in the large. In both these two areas, the works presented in the literature consider only complete information settings wherein agents have no deadlines, have the same reservation prices and discount factors, and negotiate on a single issue. ${ }^{3} \mathrm{We}$ briefly review the main contributions within these two areas.

The analysis of markets in the small has explored models wherein two agents bargain, but at least one may have an outside option. The outside option denotes the possibility for an agent of leaving the negotiation she is carrying out and gain an amount of utility. In practice, an agent has an outside option when there are several possible opponents and she can leave the negotiation she is carrying on to negotiate with a different opponent. This allows a seller agent (buyer agent) to take advantage, at every time point of the negotiation, from the competition among the buyer agents

\footnotetext{
${ }^{2}$ For the sake of completeness, we notice that action exit could be used to stop a negotiation in order to start a new negotiation with a different opponent. However, as we discuss in Section 4.1, this use raises several complications and we shall show that is not satisfactory.

${ }^{3}$ Two exceptions there are: in [12] the authors consider bargaining settings with uncertainty over agents' valuations and in [25] the authors consider settings with exogenous deadlines. However, both these works study a simplification of the alternating-offers protocol in which the seller can make an offer, whereas the buyer can only accept it or not.
} 
(seller agents) present in the market. At the equilibrium, the outside option is used if it pays better than the Rubinstein equilibrium but it is ignored otherwise. The objective of the analysis within this area is the comprehension of how the availability of the outside option (and then of competition among agents) alters the Rubinstein equilibrium. The most well known results can be found in [1, 25, 41]. In [1] the authors do not explicitly model the presence of several opponents, but they assign a specific value to the outside option. They present an experimental analysis considering three different settings, each one characterized by a specific value for the outside option. In these situations the equilibrium is unique. As the authors underline, the uniqueness of the equilibrium is of paramount importance in the presence of multiple agents such as in markets. This is particularly crucial in electronic markets, where each agent can be developed by different designers and could employ different equilibrium strategies. In [25] the authors do explicitly model the presence of several opponents and assume the matching between the agents to be random. At each time point there is a positive value of probability that an agent will meet a different opponent. They show that in this setting the agreement between the agents can be delayed. In [41] the authors show that usually markets involving a small number of agents do not yield competitive allocations because market power is retained by some traders.

In the analysis of markets in the large, the models commonly studied in the literature assume a continuum of agents who are matched at random in pairs to perform a trade of commodities. The objective of the analysis within this area is radically different from that of the previous area. More precisely, it is the analysis of the equilibrium of the market. The most well known results can be found in $[5,15-$ 17, 40]. All these works provide very simple bargaining models and they usually consider perfectly competitive situations wherein the actions made by an agent do not affect the behavior of their competitors. Different works have taken in to account different market settings: stationary markets in which there is a constant rate of agents entering the market [40], and non-stationary markets in which the rate changes during time [17]. We do not discuss the specific details of these works, as they are somewhat beside the point of the present paper. We point the interested reader to [44].

For the sake of completeness, we note that a few works on the analysis of bargaining in markets can also be found in computer science literature. However, the scope of these works is different. The problem they consider is to coordinate concurrent negotiations, e.g., see [35], and not to study the effects of the presence of the outside option in bargaining.

The results cited above are inapplicable to real-world electronic markets wherein autonomous agents carry out electronic transactions. The main reasons lay in the inadequacies of the bargaining models they consider (i.e., agents do not have deadlines, and reservation prices and discount factors are the same for all the agents) and in the unsatisfactory matching model they adopt (i.e., the agents are matched at random). In real-world settings agents have deadlines, have different utility parameters, and it is reasonable to assume that each agent can choose the opponent with whom she will negotiate. The analysis we carry out in this paper is closer to the analysis of the markets in the small. To be more precise, we propose a bargaining model which is more realistic with respect to the ones cited above and we game theoretically analyze the equilibrium strategies in the presence of complete information to derive an efficient solving algorithm. 


\subsection{The proposed model}

We extend the model presented in Section 2.1 to capture the situation where:

- $\quad$ there are $m$ buyer agents and $n$ seller agents;

- the items sold by the sellers are equal;

- $\quad$ all the sellers have exactly one item to sell;

- all the buyers are interested in buying exactly one item;

- $\quad$ agents can enter the market at any time point.

We denote by $\mathbf{b}_{i}$ the $i$-th buyer agent-her parameters will be $R P_{\mathbf{b}_{i}}, T_{\mathbf{b}_{i}}$, and $\delta_{\mathbf{b}_{i}}$-and by $\mathbf{s}_{j}$ the $j$-th seller agent-her parameters will be $R P_{\mathbf{s}_{j}}, T_{\mathbf{s}_{j}}$, and $\delta_{\mathbf{s}_{j}}$. Furthermore, each agent, both $\mathbf{b}_{i}$ and $\mathbf{s}_{j}$, will be characterized by a time point denoted by $A_{\mathbf{b}_{i}}$ and $A_{\mathbf{s}_{j}}$, respectively, where she enters the market. An agent $i$ can act in a market from $t=A_{i}$.

We design our bargaining model such that:

- at any time point non-matched agents can match non-matched opponents;

- if an agent is negotiating with an opponent, she can leave the negotiation she is carrying out to match a non-matched opponent (i.e., outside option);

- $\quad$ two agents that match at $t$ will start to negotiate at $t+d$ where $d \in \mathbb{N}, d>0$ (the value of $d$ captures a possible delay due to the negotiation platform or set by the electronic institution; we assume that the platform grants the negotiation to begin exactly at $t+d$ and then the value of $d$ is known a priori by the agents);

- given two agents matched at $t$, the agent that opens the negotiation at $t+d$ is chosen randomly with a probability .5 by the negotiation platform;

- in the presence of one buyer agent and one seller agent, agents' equilibrium strategies are exactly those in the classic alternating-offers protocol with deadlines described in Section 2.2.

In matching models, such as for instance [18], agents are split in two groups such that all the agents in the first group propose a matching and all the agents in the second group accept or reject the proposed matching. The inclusion of a matching mechanism in bargaining implicitly introduces asymmetries in the negotiation: buyer agents and sellers agents will have different available actions. In our model each buyer will announce whether or not she wants to be matched and, in the affirmative case, will announce to which seller she wants to be matched. Instead, each seller will choose which buyer to match (we report details below). Our extension of the original bargaining model develops in three points. We discuss them one by one.

(1) We introduce three actions to rule the matching between two opponents, i.e., matchable $\left(\mathbf{s}_{j}\right)$, nonmatchable, and match $\left(\mathbf{b}_{i}\right)$, and one action to allow agents to wait for the next time point, i.e., wait. The actions matchable $\left(\mathbf{s}_{j}\right)$ and nonmatchable are available at time point $t$ to the buyers that are not matched and the effect of the first one is to signal that the buyer is ready at time point $t$ to be matched by the specific seller $\mathbf{s}_{j}$, whereas the effect of the second one is to signal that she is not ready to be matched. In order for matchable $\left(\mathbf{s}_{j}\right)$ to be a valid action at time point $t, \mathbf{s}_{j}$ must be present in the market. The action $\operatorname{match}\left(\mathbf{b}_{i}\right)$ is available to the sellers and its effect is that $\mathbf{s}_{j}$ matches the buyer $\mathbf{b}_{i}$. 
Obviously, in order for match $\left(\mathbf{b}_{i}\right)$ to be a valid action for $\mathbf{s}_{j}$ at $t, \mathbf{b}_{i}$ must have chosen matchable $\left(\mathbf{s}_{j}\right)$ at the same time point. Once two agents are matched they start to negotiate after $d$ time points. The action wait is available to both buyers and sellers, and allows them to wait for the next time point. We discuss below, at point (3), when and how agents can employ these actions.

(2) We redefine action exit: if an agent chooses exit, then she leaves the market and her outcome is No Agreement. Our choice is directed at excluding agents' nonfeasible behaviors: it can be observed that if action exit does not impose agents to leave the market, then in the presence of a unique buyer and a unique seller the equilibrium strategies are different from those prescribed by Rubinstein's equilibrium. We shall show that in Section 4.1.

(3) We modify the mechanism of the game dividing each time point in two sequential stages, denoted by 1 and 2, and inhibiting and allowing actions according to the actual state an agent is in. More precisely, in a market an agent $\mathbf{b}_{i}\left(\right.$ or $\left.\mathbf{s}_{j}\right)$ can be in three different states:

- $\quad(\mathrm{S} 1) \mathbf{b}_{i}\left(\right.$ or $\left.\mathbf{s}_{j}\right)$ is non-matched,

- $\quad(\mathrm{S} 2) \mathbf{b}_{i}\left(\right.$ or $\left.\mathbf{s}_{j}\right)$ is matched with $\mathbf{s}_{j}$ (or $\mathbf{b}_{i}$ ) and is waiting to start negotiating, or

- $\quad(\mathrm{S} 3) \mathbf{b}_{i}\left(\right.$ or $\left.\mathbf{s}_{j}\right)$ is negotiating with $\mathbf{s}_{j}\left(\right.$ or $\left.\mathbf{b}_{i}\right)$.

Consider state (S1). If $\mathbf{b}_{i}$ is not matched, then her allowed actions are the following. In the first stage of every time point $t$ she can choose nonmatchable or matchable $\left(\mathbf{s}_{k}\right)$ where $\mathbf{s}_{k}$ must be present in the market at $t$. In the second stage of every time $t$ point she can choose wait or exit; this second action is allowed only if $\mathbf{b}_{i}$ has chosen nonmatchable in the first stage of $t$ : we exclude non-reasonable situations where at the same time point a buyer announces to be ready to be matched with a specific seller and subsequently leaves the market. If $\mathbf{b}_{i}$ is matched, no action is available. If $\mathbf{s}_{j}$ is not matched, then her allowed actions are the following. In the first stage of every time point $t$, no action is allowed. In the second stage of every time point $t$, she can choose wait or $\operatorname{match}\left(\mathbf{b}_{k}\right)$, where $\mathbf{b}_{k}$ must not be matched, or exit. If $\mathbf{s}_{j}$ is matched, no action is available. Consider state (S2). If $\mathbf{b}_{i}$ ( or $\mathbf{s}_{j}$ ) is matched and is waiting for starting to negotiate, no action is allowed. Consider state (S3). If $\mathbf{b}_{i}$ is negotiating, then she acts alternately and her allowed actions are exactly those in the alternatingoffers protocol discussed in the previous section, i.e., offer $(x)$, accept, and exit. If $\mathbf{s}_{j}$ is negotiating, then she acts alternately and her allowed actions are those in the alternating-offers protocol discussed in the previous section, i.e., offer $(x)$, accept, and exit, plus action match $\left(\mathbf{b}_{i}\right)$ if $\mathbf{b}_{i}$ has chosen matchable $\left(\mathbf{s}_{j}\right)$. For the sake of clarity, the actions available to agents are summarized in Table 1.

Agents' utility functions are exactly those defined in the previous section. Since every agent negotiates exclusively on one item, once a negotiation is concluded with an acceptance, the two negotiators leave the market. When $m>n$ (or $m<n) m-n$ buyer agents (or $n-m$ seller agents) will not reach any agreement and therefore their outcomes will be NoAgreement.

We discuss an example of possible agents' behaviors in the above protocol. Consider a setting with four buyers (i.e., $\left.\mathbf{b}_{1}, \mathbf{b}_{2}, \mathbf{b}_{3}, \mathbf{b}_{4}\right)$ and one seller (i.e., s) with $T_{\mathbf{b}_{1}}=4, T_{\mathbf{b}_{2}}=2, T_{\mathbf{b}_{3}}=4, T_{\mathbf{b}_{4}}=4$, and $T_{\mathbf{s}}=6$, and $A_{\mathbf{b}_{1}}=0, A_{\mathbf{b}_{2}}=0, A_{\mathbf{b}_{3}}=0, A_{\mathbf{b}_{4}}=$ 1 , and $A_{\mathbf{s}}=0$ (we do not specify the value of the other parameters, being unnecessary 
Table 1 Actions available to an agent $i$ in function of her state

\begin{tabular}{|c|c|c|c|c|}
\hline State & Stage & Agent & Time points & Available actions \\
\hline \multirow[t]{3}{*}{$\begin{array}{l}\mathbf{b}_{i}\left(\mathbf{s}_{j}\right) \text { is not } \\
\text { matched }\end{array}$} & 1 & $\mathbf{b}_{i}$ & Any & $\begin{array}{l}\text { nonmatchable, } \\
\text { matchable }\left(\mathbf{s}_{k}\right)\end{array}$ \\
\hline & 2 & $\mathbf{b}_{i}$ & Any & $\begin{array}{l}\text { wait, exit if } \mathbf{b}_{i} \text { has } \\
\text { made nonmatchable }\end{array}$ \\
\hline & & $\mathbf{s}_{j}$ & Any & $\begin{array}{l}\text { wait, } \text { match }\left(\mathbf{b}_{i}\right) \text { if } \mathbf{b}_{i} \text { has } \\
\text { made matchable }\left(\mathbf{s}_{j}\right), \text { exit }\end{array}$ \\
\hline \multirow{2}{*}{$\begin{array}{l}\mathbf{b}_{i}\left(\mathbf{s}_{j}\right) \text { is } \\
\text { negotiating }\end{array}$} & 2 & $\mathbf{b}_{i}$ & Alternately & offer $(x)$, accept, exit \\
\hline & & $\mathbf{s}_{j}$ & Alternately & $\begin{array}{l}\text { offer }(x), \text { accept, exit, } \\
\qquad \text { match }\left(\mathbf{b}_{i}\right) \text { if } \mathbf{b}_{i} \text { has made } \\
\quad \text { matchable }\left(\mathbf{s}_{j}\right)\end{array}$ \\
\hline
\end{tabular}

for our example). In the first stage of time point $t=0, \mathbf{b}_{1}, \mathbf{b}_{2}$, and $\mathbf{b}_{3}$ can choose matchable(s) or nonmatchable (notice that $\mathbf{b}_{4}$ has not entered the market yet). Suppose that $\mathbf{b}_{1}$ and $\mathbf{b}_{2}$ choose matchable(s), whereas $\mathbf{b}_{3}$ chooses nonmatchable. In the second stage of time point $t=0$, s can choose wait or $\operatorname{match}\left(\mathbf{b}_{i}\right)$ where $i \in\{1,2\}$, $\mathbf{b}_{1}$ and $\mathbf{b}_{2}$ can choose wait, and $\mathbf{b}_{3}$ can choose wait or exit. Suppose that $\mathbf{s}$ chooses $\operatorname{match}\left(\mathbf{b}_{1}\right)$ and that $\mathbf{b}_{1}, \mathbf{b}_{2}$, and $\mathbf{b}_{3}$ choose wait. Now, $\mathbf{b}_{1}$ and $\mathbf{s}$ are matched and they will start to negotiate at $t=d$. Suppose that $d=1$. At $t=1$ the platform randomly selects the agent that opens the negotiation. Suppose that she is $\mathbf{s}$. Furthermore, at $t=1 \mathbf{b}_{4}$ enters the market. In the first stage of time point $t=1, \mathbf{b}_{2}, \mathbf{b}_{3}$, and $\mathbf{b}_{4}$ can choose matchable(s) or nonmatchable. Suppose that $\mathbf{b}_{2}$ chooses nonmatchable and that $\mathbf{b}_{3}$ and $\mathbf{b}_{4}$ choose matchable(s). In the second stage of time point $t=1$, $\mathbf{s}$ can choose $\operatorname{offer}(\cdot)$, exit, or match $\left(\mathbf{b}_{i}\right)$ where $i \in\{3,4\}, \mathbf{b}_{2}$ can choose wait or exit, and $\mathbf{b}_{3}$ and $\mathbf{b}_{4}$ can choose wait. Suppose that $\mathbf{s}$ chooses match $\left(\mathbf{b}_{3}\right)$, that $\mathbf{b}_{2}$ chooses exit, and that $\mathbf{b}_{3}$ and $\mathbf{b}_{4}$ choose wait. Therefore $\mathbf{s}$ leaves the negotiation she is carrying out with $\mathbf{b}_{1}$ and will start to negotiate at $t=1+d=2$ with $\mathbf{b}_{3}$.

For the sake of clarity, we introduce the notations we use in the following sections. We denote by $\Gamma\left(t,\left\{\mathbf{b}_{i}, \mathbf{s}_{j}\right\}, \iota(t)\right)$ the negotiation starting at $t$ where $\mathbf{b}_{i}$ and $\mathbf{s}_{j}$ are matched and $\iota(t)$ is the agent between $\mathbf{b}_{i}$ and $\mathbf{s}_{j}$ that acts at $t$. We denote by $\sigma_{\mathbf{b}_{i}}^{*}\left(t, \mathbf{s}_{j}\right)$ the optimal strategy of $\mathbf{b}_{i}$ in $\Gamma\left(t,\left\{\mathbf{b}_{i}, \mathbf{s}_{j}\right\}, \iota(t)=\mathbf{b}_{i}\right)$ at $t$, i.e., when $\mathbf{b}_{i}$ is negotiating with $\mathbf{s}_{j}$ and $\mathbf{b}_{i}$ is the agent that acts at $t$. We denote by $x_{\mathbf{b}_{i}}^{*}\left(t, \mathbf{s}_{j}\right)$ the optimal offer, if it exists, of $\mathbf{b}_{i}$ in the negotiation with $\mathbf{s}_{j}$ at $t$. Analogously, we define $\sigma_{\mathbf{s}_{j}}^{*}\left(t, \mathbf{b}_{i}\right)$ and $x_{\mathbf{s}_{j}}^{*}\left(t, \mathbf{b}_{i}\right)$. We introduce the concept of equivalent value $e_{\mathbf{b}_{i}}\left(\sigma, t, \mathbf{s}_{j}\right)$ of an action $\sigma$ performed by $\mathbf{b}_{i}$ at $t$ in $\Gamma\left(t,\left\{\mathbf{b}_{i}, \mathbf{s}_{j}\right\}, \iota(t)=\mathbf{b}_{i}\right)$ : we define it as the value such that $U_{\mathbf{b}_{i}}\left(e_{\mathbf{b}_{i}}\left(\sigma, t, \mathbf{s}_{j}\right), t\right)=E U_{\mathbf{b}_{i}}\left(\sigma, t, \mathbf{s}_{j}\right)$ where $E U_{\mathbf{b}_{i}}$ is the utility expected by $\mathbf{b}_{i}$ from making $\sigma$ at $t$ in $\Gamma\left(t,\left\{\mathbf{b}_{i}, \mathbf{s}_{j}\right\}, \iota(t)=\mathbf{b}_{i}\right)$. We denote by $e_{\mathbf{b}_{i}}^{*}\left(t, \mathbf{s}_{j}\right)$ the equivalent value of the optimal action of $\mathbf{b}_{i}$ at $t$ in $\Gamma\left(t,\left\{\mathbf{b}_{i}, \mathbf{s}_{j}\right\}, \iota(t)=\mathbf{b}_{i}\right)$. Analogously, we define $e_{\mathbf{s}_{j}}\left(\sigma, t, \mathbf{b}_{i}\right)$ and $e_{\mathbf{s}_{j}}^{*}\left(t, \mathbf{b}_{i}\right)$. Finally, we denote by $\bar{T}_{\mathbf{b}_{i}, \mathbf{s}_{j}}=\min \left\{T_{\mathbf{b}_{1}}, T_{\mathbf{s}_{j}}\right\}$ and by $\bar{A}_{\mathbf{b}_{i}, \mathbf{s}_{j}}=\max \left\{A_{\mathbf{b}_{1}}, A_{\mathbf{s}_{j}}\right\}$.

We present our results by degrees as follows. In Section 4 we study the settings wherein seller agents' outside option is inhibited. In Section 5 we study the settings wherein there is a unique seller agent and this can exploit the outside option. The settings where there are multiple seller agents and they can exploit the outside option will be discussed in future works. 


\section{Analysis without outside option}

In this section we inhibit the seller agents' ability to exploit the outside option. They cannot use action match $(\cdot)$ when they are matched. We study initially (Section 4.1) the base case in which there is a unique buyer agent and a unique seller agent and subsequently (Section 4.2) the general case wherein there are multiple buyer agents and multiple seller agents.

\subsection{The base case: one buyer and one seller}

The analysis of this case has two aims. The first one is to show that the agents' equilibrium strategies in the negotiation protocol discussed in the previous section collapse to the ones in the original protocol discussed in Section 2 and therefore our protocol is well defined. The second one is to show that the redefinition of action exit as we accomplished in the previous section is necessary to avoid non-reasonable agents' strategies.

We consider the setting in which there is a unique buyer agent $\mathbf{b}_{1}$ and a unique seller agent $\mathbf{s}_{1}$ with $R P_{\mathbf{b}_{1}}>R P_{\mathbf{s}_{1}}$ and $\bar{A}_{\mathbf{b}_{1}, \mathbf{s}_{1}}+d<\bar{T}_{\mathbf{b}_{1}, \mathbf{s}_{1}}$. These last conditions assure that the agents expect strictly positive utility from starting a negotiation. We can state the following lemma, whose proof is omitted, being trivial.

Lemma 4.1 Once two opponents have started to negotiate, they employ the equilibrium strategies prescribed in Section 2.2.

The sequences of the optimal offers $x_{\mathbf{s}_{1}}^{*}\left(t, \mathbf{b}_{1}\right)$ and $x_{\mathbf{b}_{1}}^{*}\left(t, \mathbf{s}_{1}\right)$ are to be computed as prescribed in Section 2.2. They are:

$$
\begin{aligned}
& -\quad x_{\mathbf{b}_{1}}^{*}\left(t, \mathbf{s}_{1}\right)= \begin{cases}R P_{\mathbf{s}_{1}} & \text { if } t=\bar{T}_{\mathbf{b}_{1}, \mathbf{s}_{1}}-1, \\
\left(x_{\mathbf{s}_{1}}^{*}\left(t+1, \mathbf{b}_{1}\right)\right)_{\leftarrow \mathbf{s}_{1}} & \text { otherwise }\end{cases} \\
& -\quad x_{\mathbf{s}_{1}}^{*}\left(t, \mathbf{b}_{1}\right)= \begin{cases}R P_{\mathbf{b}_{1}} & \text { if } t=\bar{T}_{\mathbf{b}_{1}, \mathbf{s}_{1}}-1 \\
\left(x_{\mathbf{b}_{1}}^{*}\left(t+1, \mathbf{s}_{1}\right)\right)_{\leftarrow \mathbf{b}_{1}} & \text { otherwise }\end{cases}
\end{aligned}
$$

The utility expected by $\mathbf{b}_{1}$ from starting a negotiation with $\mathbf{s}_{1}$ at $t$ is:

$$
E U_{\mathbf{b}_{1}}\left(\mathbf{s}_{1}\right)=\frac{1}{2} U_{\mathbf{b}_{1}}\left(x_{\mathbf{b}_{1}}^{*}\left(t, \mathbf{s}_{1}\right), t+1\right)+\frac{1}{2} U_{\mathbf{s}_{1}}\left(x_{\mathbf{s}_{1}}^{*}\left(t, \mathbf{b}_{1}\right), t+1\right) .
$$

Indeed, with .5 probability that $\mathbf{b}_{1}$ opens the negotiation offering $x_{\mathbf{b}_{1}}^{*}\left(t, \mathbf{s}_{1}\right)$ and with .5 probability that $\mathbf{s}_{1}$ opens the negotiation offering $x_{\mathbf{s}_{1}}^{*}\left(t, \mathbf{b}_{1}\right)$. The utility expected by $\mathbf{s}_{1}$ from starting a negotiation with $\mathbf{b}_{1}$ at $t$ is defined analogously. We can now state the following lemma.

Lemma 4.2 If $\mathbf{b}_{1}$ and $\mathbf{s}_{1}$ are both present in the market at $t<\bar{T}-d$ and they are not matched, then they prefer to immediately match themselves rather than to do it subsequently or not to do it at all.

Proof We study $\mathbf{b}_{1}$ 's optimal actions ( $\mathbf{s}_{1}$ 's ones can be similarly studied). We consider the case with $d=1$; when $d>1$ the proof is analogous. 
We need to prove that $\mathbf{b}_{1}$ 's expected utility of starting a negotiation at $t$ is larger than her expected utility of starting a negotiation at $t+1$. In formula, we need to prove that:

$$
\begin{aligned}
& \left(R P_{\mathbf{b}_{1}}-\frac{1}{2} x_{\mathbf{b}_{1}}^{*}\left(t, \mathbf{s}_{1}\right)-\frac{1}{2} x_{\mathbf{s}_{1}}^{*}\left(t, \mathbf{b}_{1}\right)\right) . \\
& \left(\delta_{\mathbf{b}_{1}}\right)^{t+1}>\left(R P_{\mathbf{b}_{1}}-\frac{1}{2} x_{\mathbf{b}_{1}}^{*}\left(t+1, \mathbf{s}_{1}\right)-\frac{1}{2} x_{\mathbf{s}_{1}}^{*}\left(t+1, \mathbf{b}_{1}\right)\right) \cdot\left(\delta_{\mathbf{b}_{1}}\right)^{t+2} .
\end{aligned}
$$

We consider the worst case, i.e., when $\delta_{\mathbf{s}_{1}}=1$. We show that in the worst case the above inequality holds (and therefore always holds). When $\delta_{\mathbf{s}_{1}}=1$ we have that $x_{\mathbf{b}_{1}}^{*}\left(t, \mathbf{s}_{1}\right)=x_{\mathbf{s}_{1}}^{*}\left(t+1, \mathbf{b}_{1}\right)$ and $x_{\mathbf{s}_{1}}^{*}\left(t, \mathbf{b}_{1}\right)=\left(x_{\mathbf{b}_{1}}^{*}(t+1)\right)_{\leftarrow \mathbf{b}_{1}}$. The above inequality can be written as:

$$
\frac{1}{2} \cdot\left(1-\delta_{\mathbf{b}_{1}}\right) \cdot\left(R P_{\mathbf{b}_{1}}-x_{\mathbf{s}_{1}}^{*}\left(t+1, \mathbf{b}_{1}\right)\right)>0 .
$$

Since $R P_{\mathbf{b}_{1}}-x_{\mathbf{s}_{1}}^{*}\left(t+1, \mathbf{b}_{1}\right)>0$ by construction and $1-\delta_{\mathbf{b}_{1}}>0$ by definition, the above inequality holds for every value of the parameters.

From Lemmas 4.1 and 4.2, the following theorem trivially follows.

Theorem 4.3 In presence of one buyer and one seller, agents equilibrium strategies at $t \geq \max \left\{A_{\mathbf{b}_{1}}, A_{\mathbf{s}_{1}}\right\}$ are:

$$
\begin{aligned}
& \sigma_{\mathbf{b}_{1}}(t)= \begin{cases}\text { if } \mathbf{b}_{1} \text { is matched } & \text { strategies prescribed in Section 2.2 } \\
\text { if } \mathbf{b}_{1} \text { is non-matched } & \left\{\begin{array} { l } 
{ t < \overline { T } - d } \\
{ \overline { T } - d \leq t }
\end{array} \left\{\begin{array}{lll}
\text { stage } 1 & \text { matchable }\left(\mathbf{s}_{1}\right) \\
\text { stage 2 } & \text { wait }
\end{array}\right.\right. \\
\text { stage 1 } & \text { nonmatchable } \\
\text { stage 2 } & \text { exit }\end{cases} \\
& \sigma_{\mathbf{s}_{1}}(t)= \begin{cases}\text { if } \mathbf{s}_{1} \text { is matched } & \text { strategies prescribed in Section } 2.2 \\
\text { if } \mathbf{s}_{1} \text { is non-matched } & \left\{\begin{array}{ll}
t<\bar{T}-d \\
\bar{T}-d \leq t
\end{array}\right\} \text { stage } 2 \quad \text { match }\left(\mathbf{b}_{2}\right) . \\
\text { stage } 2 & \text { exit }\end{cases}
\end{aligned}
$$

At the equilibrium, if $\bar{A}_{\mathbf{b}_{1}, \mathbf{s}_{1}}+d<\bar{T}_{\mathbf{b}_{1}, \mathbf{s}_{1}}$, then at $t=\bar{A}_{\mathbf{b}_{1}, \mathbf{s}_{1}}$ agent $\mathbf{b}_{1}$ will make $\operatorname{match}\left(\mathbf{s}_{1}\right)$ and agent $\mathbf{s}_{1}$ will make $\operatorname{match}\left(\mathbf{b}_{1}\right)$. They will start to negotiate at $t=$ $\bar{A}_{\mathbf{b}_{1}, \mathbf{s}_{1}}+d$ and they will reach an agreement at $t=\bar{A}_{\mathbf{b}_{1}, \mathbf{s}_{1}}+d+1$.

We show now that, if action exit does not impose agents to leave the negotiation platform, then Lemma 4.1 does not hold and therefore, in the presence of a unique buyer and a unique seller, agents' equilibrium strategies are different from those in the original protocol presented in Section 2.2.

Consider the bargaining setting with one buyer agent $\mathbf{b}_{1}$ and one seller agent $\mathbf{s}_{1}$, with $d=1$, and where both agents enter the market at $t=0$ : agents' optimal offers are reported in Fig. 3. It can be observed that agents will immediately match themselves and then they will start to negotiate at $t=1$. If agents can make exit without leaving the market, then they will make it at $t=1$. Consider the 

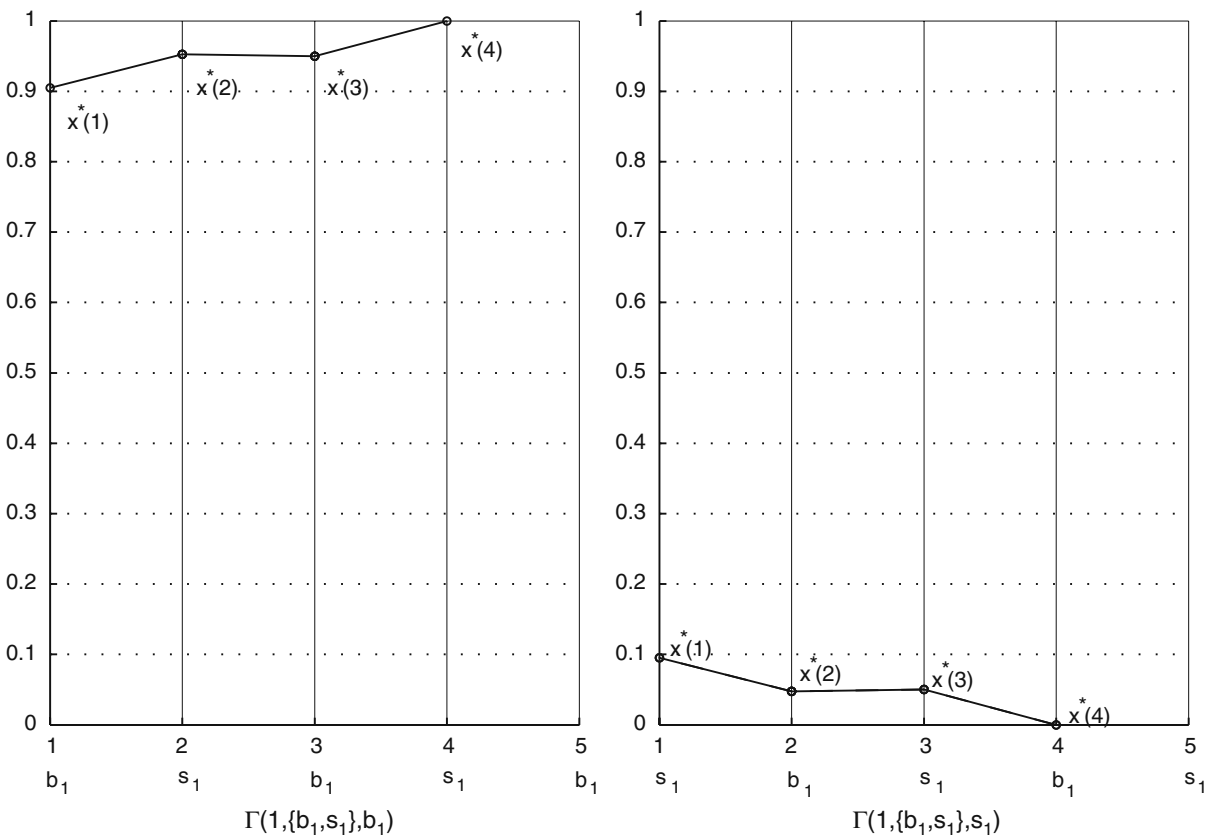

Fig. 3 Agents' optimal offers when $R P_{\mathbf{b}_{1}}=1, \delta_{\mathbf{b}_{1}}=.95, T_{\mathbf{b}_{1}}=5, R P_{\mathbf{s}_{1}}=0, \delta_{\mathbf{s}_{1}}=.95, T_{\mathbf{s}_{1}}=12$, and $d=1$

negotiation in which $\iota(1)=\mathbf{s}_{1}: \mathbf{s}_{1}$ opens the negotiation. If $\mathbf{s}_{1}$ employs the strategies prescribed in Section 2.2, i.e., $\operatorname{offer}\left(x_{\mathbf{s}_{1}}^{*}\left(1, \mathbf{b}_{1}\right)\right)$ with $x_{\mathbf{s}_{1}}^{*}\left(1, \mathbf{b}_{1}\right) \simeq .1$, then she gains $U_{\mathbf{s}_{1}}\left(x_{\mathbf{s}_{1}}^{*}\left(1, \mathbf{b}_{1}\right), 0\right) \simeq .1$. If $\mathbf{s}$ makes exit and subsequently match $\left(\mathbf{b}_{1}\right)$ at $t=2$, then she gains $\simeq .45$. To be exact, the computation of the expected utility of making exit at $t=1$ and subsequently $\operatorname{match}\left(\mathbf{b}_{1}\right)$ at $t=2$ is: with probability $.5 \mathbf{s}_{1}$ acts at $t=3$ offering $x_{\mathbf{s}_{1}}^{*}\left(3, \mathbf{b}_{1}\right)=.05$ and with probability $.5 \mathbf{b}_{1}$ acts at $t=3$ offering $x_{\mathbf{b}_{1}}^{*}\left(1, \mathbf{s}_{1}\right)=$ .95; since these agreements are reached at $t=4$, their utility must be discounted by $\left(\delta_{\mathbf{s}_{1}}\right)^{2}$. Notice that agents prefer to offer rather than to exit at $t=3$ (otherwise agents would not reach any agreement).

\subsection{The general case: multiple buyers and multiple sellers}

The analysis of the setting with multiple buyers and multiple sellers is not straightforward. This is due to the intrinsic complexity of the game: it is essentially a multistage game wherein $m+n$ agents concurrently act and the game changes at each stage. Nevertheless, Lemma 4.1 (i.e, once two opponents are matched, they negotiate as prescribed in Section 2.2) and Lemma 4.2 (i.e., two opponents prefer to start to negotiate immediately rather than to wait for some time points and subsequently start to negotiate) reduces this problem to one of matching. We recall that in this case a matching is an assignment of buyers to sellers such that each buyer is assigned to at most one seller and vice versa. Among all the possible matchings, we need to find a matching that is of equilibrium. Such a matching is a stable matching that is 
optimal for the buyer agents, see [18]. Formally, a matching is said to be stable if there is not any $\mathbf{b}_{i}, \mathbf{b}_{j}, \mathbf{s}_{k}, \mathbf{s}_{l}$ such that:

- $\mathbf{b}_{i}$ is matched to $\mathbf{s}_{k}$,

- $\quad \mathbf{b}_{j}$ is matched to $\mathbf{s}_{l}$,

- $\quad \mathbf{b}_{i}$ prefers $\mathbf{s}_{l}$ than $\mathbf{s}_{k}$ and $\mathbf{s}_{l}$ prefers $\mathbf{b}_{i}$ than $\mathbf{s}_{k}$.

A stable matching can be found by employing the Gale-Shapley's stable marriage algorithm [18]. Gale and Shapley consider the problem of matching males and females given that each male has a preference ordering over the females and each female has a preference ordering over the males. They provide two versions of their algorithm. In the first one, a male proposes to the female and the resulting matching is optimal for the male. In the second one, vice versa. In what follows we present an algorithm based on Gale-Shapley's algorithm to address our specific case. The algorithm develops in three steps. For the sake of presentation, we initially consider the case in which $m=n$ and $\min _{i}\left\{R P_{\mathbf{b}_{i}}\right\}>\max _{j}\left\{R P_{\mathbf{s}_{j}}\right\}$ and subsequently we consider the general case. (This is because Gale-Shapley's algorithm requires that the number of buyers is equal to the number of sellers and that every agent prefers to be matched rather than not to be.)

Step 1 In this step the utility expected by every agent from negotiating with every possible opponent is computed. That is, for every buyer agent $\mathbf{b}_{i}$ the expected utility from negotiating with every possible $\mathbf{s}_{j}$ must be computed, and vice $v e r s a$. In formula, the utility expected by $\mathbf{b}_{i}$ from negotiating with $\mathbf{s}_{j}$ is:

$$
E U_{\mathbf{b}_{1}}\left(\mathbf{s}_{j}\right)=\left\{\begin{array}{cl}
\frac{1}{2} U_{\mathbf{b}_{1}}\left(x_{\mathbf{b}_{1}}^{*}\left(\bar{A}_{\mathbf{b}_{i}, \mathbf{s}_{j}}+d\right), \bar{A}_{\mathbf{b}_{i}, \mathbf{s}_{j}}+d+1\right)+ & \text { if } \bar{A}_{\mathbf{b}_{i}, \mathbf{s}_{j}}+d<\bar{T}_{\mathbf{b}_{i}, \mathbf{s}_{j}} \\
\quad+\frac{1}{2} U_{\mathbf{b}_{1}}\left(x_{\mathbf{s}_{1}}^{*}\left(\bar{A}_{\mathbf{b}_{i}, \mathbf{s}_{j}}+d\right), \bar{A}_{\mathbf{b}_{i}, \mathbf{s}_{j}}+d+1\right) & \text { otherwise }
\end{array}\right.
$$

The utility expected by $\mathbf{s}_{j}$ from negotiating with $\mathbf{b}_{i}$ is defined analogously. In order to compute the agents' expected utilities, we need to compute the sequences of the agents' optimal offers in all the possible negotiations. Exactly, the number of backward induction constructions to be built are $2 \cdot m \cdot n$ and therefore the computational time required to calculate all the optimal offer sequences is $O\left(m \cdot n \cdot \max _{i, j}\left\{\bar{T}_{\mathbf{b}_{i}, \mathbf{s}_{j}}\right\}\right)$.

Step 2 On the basis of the agents' expected utilities, agents' preference ordering concerning the opponents to whom they would be matched are found. More precisely, for every buyer $\mathbf{b}_{i}$ (and for every seller $\mathbf{s}_{j}$ ) we need to sort the sellers (the buyers) from the one who gives $\mathbf{b}_{i}$ the maximum expected utility to the one who gives $\mathbf{b}_{i}$ the minimum expected utility. An example is the following:

$$
\left(\begin{array}{c}
\mathbf{b}_{1}: \mathbf{s}_{2} \succ \mathbf{s}_{3} \succ \mathbf{s}_{1} \succ \mathbf{s}_{5} \succ \mathbf{s}_{6} \succ \ldots \\
\mathbf{b}_{2}: \mathbf{s}_{7} \succ \mathbf{s}_{1} \succ \mathbf{s}_{3} \succ \mathbf{s}_{4} \succ \mathbf{s}_{2} \succ \ldots \\
\cdots \\
\quad \ldots \\
\mathbf{b}_{m}: \mathbf{s}_{7} \succ \mathbf{s}_{5} \succ \mathbf{s}_{2} \succ \mathbf{s}_{1} \succ \mathbf{s}_{6} \succ \ldots
\end{array}\right)
$$

(this is to say that $\mathbf{b}_{1}$ prefers to match $\mathbf{s}_{2}$ than $\mathbf{s}_{3}$, prefers to match $\mathbf{s}_{3}$ than $\mathbf{s}_{1}$, and so on). The sellers' preference ordering can be found analogously. The computational time required to build agents' preference orderings is $O(m \cdot n)$. 
Step 3 On the basis of the agents' preferences we can apply Gale-Shapley's algorithm. We reciew their algorithm in our specific case.

Definition 4.4 (Gale-Shapley stable marriage algorithm) The algorithm is iterative. Let $k=1$.

1. Each buyer agent that is not matched proposes to the $k$-th top-ranked seller agent in her preferences.

2. Each seller agent keeps her top-ranked proposal among the ones she has received (including ones from previous iterative steps). Two cases are possible. In the first case each seller agent receives only one proposal. In this case, each buyer agent matches a seller agent and then the algorithm terminates. In the second case some seller agents receive more than one proposal. In this case, these agents choose the buyer agent they prefer and the algorithm goes to step 3 .

3. The value of $k$ is increased by 1 and the algorithm goes to step 1 .

The Gale-Shapley's work shows that:

- the algorithm always terminates (the proof is trivial, the number of buyers and sellers being finite),

- a stable matching always exists,

- the produced matching is optimal for the buyer agents.

Furthermore, the stable matching that is optimal for the buyers is unique except when agents are indifferent to two or more matching agents. For instance, when $\mathbf{b}_{1}$ 's preferences prescribe $\mathbf{s}_{1} \sim \mathbf{s}_{2} \succ \mathbf{s}_{3} \succ \ldots \succ \mathbf{s}_{n}$. This happens when the utility expected by $\mathbf{b}_{1}$ from matching $\mathbf{s}_{1}$ is equal to the one expected from matching $\mathbf{s}_{2}$. Generally, this happens only for a null-measure subset of the space of the parameters and therefore is negligible. ${ }^{4}$

To capture the cases in which $m \neq n$ and $\min _{i}\left\{R P_{\mathbf{b}_{i}}\right\}<\max _{j}\left\{R P_{\mathbf{s}_{j}}\right\}$, the algorithm must be extended. We proceed by degrees. We consider the situations where $m \neq n$. As is underlined by Gale and Shapley, when $m \neq n$ we need to introduce fictitious agents. Suppose that $m>n$ (the case in which $m<n$ can be treated analogously), $m-n$ buyer agents cannot be matched. In order for Gale-Shapley's algorithm to work correctly, we need to introduce $m-n$ fictitious seller agents such that the utility expected by a buyer agent from negotiating with one of these agents is zero. The preferences of the fictitious agents can be anything. If the matching produced by the above algorithm prescribes $\mathbf{b}_{i}$ to be matched with a fictitious seller agent, then (in practice) $\mathbf{b}_{i}$ will not be matched.

We consider the cases where $\min _{i}\left\{R P_{\mathbf{b}_{i}}\right\}<\max _{j}\left\{R P_{\mathbf{s}_{j}}\right\}$. In these situations agents could prefer not to be matched rather than to be matched. For instance, when $R P_{\mathbf{b}_{1}}=.5, R P_{\mathbf{b}_{2}}=.6, R P_{\mathbf{b}_{3}}=.4$ and $R P_{\mathbf{s}_{1}}=.2, R P_{\mathbf{s}_{2}}=.3, R P_{\mathbf{s}_{3}}=.7$, s prefers not to be matched rather than to be matched. Similarly to what we have done previously, these cases can be addressed by introducing fictitious agents. We need to introduce $m$ fictitious seller agents and $n$ fictitious buyer agents. In this way, the overall number

\footnotetext{
${ }^{4}$ In measure theory, a null measure set is a set that is negligible for the purposes of the measure in question [23]. As commonly done in the literature to study sets in Euclidean $n$-space $\mathbb{R}^{n}$, we use Lebesgue measure.
} 
of buyer agents is equal to the overall number of seller agents, i.e., $m+n$. As above, the utility expected by an agent from negotiating with a fictitious agent is zero and the preferences of the fictitious agents can be anything. Notice that in the case $\max _{i}\left\{R P_{\mathbf{b}_{i}}\right\}<\min _{j}\left\{R P_{\mathbf{s}_{j}}\right\}$, the matching produced by the above algorithm prescribes that all the buyers agents will be matched to the fictitious seller agents and all the seller agents will be matched to the fictitious buyer agents. The number of iterations in Gale-Shapley's algorithm in the worst case is $O\left((m+n)^{2}\right)$.

In summary, the problem of bargaining when the outside option is inhibited can be reduced in polynomial time to the problem of finding a stable matching. With singleissue negotiation the computational complexity is therefore $O\left(m \cdot n \cdot \max _{i, j}\left\{\bar{T}_{\mathbf{b}_{i}, \mathbf{s}_{i}}\right\}+\right.$ $\left.(m+n)^{2}\right)$. The presence of multiple issues exclusively affects the computation of Step 1 and therefore the computational complexity is $O\left(l \cdot m \cdot n \cdot \max _{i, j}\left\{\bar{T}_{\mathbf{b}_{i}, \mathbf{s}_{j}}\right\}+(m+\right.$ $n)^{2}$ ).

\section{Analysis with outside option and one-sided competition}

In this section we consider the setting where there is a unique seller and she can exploit the outside option. We game theoretically analyze the base case wherein there are two buyers, $\mathbf{b}_{1}$ and $\mathbf{b}_{2}$, with $R P_{\mathbf{b}_{1}}=R P_{\mathbf{b}_{2}}=R P_{\mathbf{b}}>R P_{\mathbf{s}}, T_{\mathbf{b}_{1}} \leq T_{\mathbf{b}_{2}}$, and $A_{\mathbf{b}_{1}}=-d, A_{\mathbf{b}_{2}}=-d .^{5}$ We discuss in Appendix A the general case. At first (Section 5.1) we provide agents' equilibrium strategies, subsequently (Section 5.2) we discuss equilibrium uniqueness.

\subsection{Equilibrium strategies in the base case}

The equilibrium strategies can be found by applying the backward induction method, but, due to the complexity of the this setting, its application is much involved. (We write in this section the bottom-line of the application of the backward induction method and we report the pertinent proofs in Appendix B.) Without loss of generality we suppose that $\mathbf{s}$ will enter the market at $t=-d$. Therefore, she will choose match $\left(\mathbf{b}_{i}\right)$ with $i \in\{1,2\}$ at $t=-d$ and subsequently $\mathbf{s}$ and the matched $\mathbf{b}_{i}$ will begin to negotiate at $t=0 .{ }^{6}$ First we find backwards the optimal strategies of the agents from $t=\bar{T}$, where $\bar{T}=\min \left\{\max \left\{T_{\mathbf{b}_{1}}, T_{\mathbf{b}_{2}}\right\}, T_{\mathbf{s}}\right\}$, to $t=0$ in all the possible subgames that the agents can play in and subsequently we determine how $\mathbf{b}_{i}$ can be optimally matched by $\mathbf{s}$ at $t=-d$. More precisely, with $\mathbf{b}_{1}$ and $\mathbf{b}_{2}$ we must provide agents' optimal strategies in all the following subgames: $\Gamma\left(t, \mathbf{b}_{1}, \mathbf{b}_{1}\right), \Gamma\left(t, \mathbf{b}_{1}, \mathbf{s}\right), \Gamma\left(t, \mathbf{b}_{2}, \mathbf{b}_{2}\right)$, and $\Gamma\left(t, \mathbf{b}_{2}, \mathbf{s}\right)$ for any $0 \leq t \leq \bar{T}$.

We firstly consider the non-matched buyers' optimal actions in the first stage of any time point. Each buyer $\mathbf{b}_{i}$ will choose matchable at $t$ if $t<\min \left\{T_{\mathbf{b}_{i}}, \bar{T}\right\}-d$. As we shall show below, because $R P_{\mathbf{b}_{1}}=R P_{\mathbf{b}_{2}}$ each $\mathbf{b}_{i}$ expects a strictly positive utility from starting a negotiation at $t<\min \left\{T_{\mathbf{b}_{i}}, \bar{T}\right\}$. Instead, agent $\mathbf{b}_{i}$ expects zero utility from starting a negotiation at $t \geq T_{\mathbf{b}_{i}}$ or at $t \geq \bar{T}$. Indeed, if $\mathbf{b}_{i}$ starts to

\footnotetext{
${ }^{5}$ For the sake of simplicity, we use $\Gamma\left(t, \mathbf{b}_{i}, \iota(t)\right)$ in the place of $\Gamma\left(t,\left\{\mathbf{b}_{i}, \mathbf{s}\right\}, \iota(t)\right)$, and $x_{\mathbf{b}_{i}}(t), \sigma_{\mathbf{b}_{i}}(t)$ in the place of $x_{\mathbf{b}_{i}}^{*}(t, \mathbf{s}), e_{\mathbf{b}_{i}}(t, \mathbf{s})$, respectively.

${ }^{6} \mathrm{By}$ extending the analysis discussed in Section 4.1, it can be shown that the agents prefer to immediately match their opponent rather than to wait for some time points before matching them.
} 
negotiate at $t \geq T_{\mathbf{b}_{i}}$, then she will make exit, reaching NoAgreement and gaining thus 0. Similarly, if $\mathbf{b}_{i}$ starts to negotiate at $t \geq \bar{T}$, she will gain 0 . As a result, $\mathbf{b}_{i}$ is indifferent between making matchable(s) and nonmatchable at $t \geq \min \left\{T_{\mathbf{b}_{i}}, \bar{T}\right\}-d$. This indifference is problematic, since it leads to the existence of multiple equilibria with different equilibria outcomes. In this section we assume that $\mathbf{b}_{i}$ will choose nonmatchable. ${ }^{7}$ Therefore, each non-matched buyer $\mathbf{b}_{i}$ will choose nonmatchable at $t$ if $\min \left\{T_{\mathbf{b}_{i}}, \bar{T}\right\}-d \leq t$. We consider now the non-matched buyers' actions in the second stage of any time point. Each non-matched buyer $\mathbf{b}_{i}$ will choose wait at $t<\min \left\{T_{\mathbf{b}_{i}}, \bar{T}\right\}-d$ and exit from $t=\min \left\{T_{\mathbf{b}_{i}}, \bar{T}\right\}-d$. (Notice that on the equilibrium path a non-matched buyer $\mathbf{b}_{i}$ could also choose wait at $\min \left\{T_{\mathbf{b}_{i}}, \bar{T}\right\}-d \leq t$ instead of exit, but this fact does not alter the equilibrium outcome: such a buyer will never be matched at $\min \left\{T_{\mathbf{b}_{i}}, \bar{T}\right\}-d \leq t$.)

We consider now the optimal actions of the matched agents in the second stage of each time point during the negotiation. We preliminarily introduce the principle on which the choice rules employed by the agents at the equilibrium are based and subsequently we discuss these choice rules in detail. As we shall show below, the equilibrium strategies of $\mathbf{b}_{i}$ can be satisfactorily defined on the basis of the sequence $x_{\mathbf{b}_{i}}^{*}(t)$ similarly to the equilibrium strategies discussed in Section 2.2: if $\mathbf{b}_{i}$ receives an offer $x \leq\left(x_{\mathbf{b}_{i}}^{*}(t)\right)_{\leftarrow \mathbf{b}_{i}}$ at $t$, then she accepts it, otherwise she rejects it to offer $x_{\mathbf{b}_{i}}^{*}(t)$. And $x_{\mathbf{b}_{i}}^{*}(t)$ is such that it will be accepted by $\mathbf{s}$ at $t+1$. (Obviously, the values of $x_{\mathbf{b}_{i}}^{*}(t)$ in the presence of multiple buyers will be different from those prescribed in Section 2.2.)

On the contrary, since at some time points the optimal offer $x_{\mathbf{s}}^{*}\left(t, \mathbf{b}_{i}\right)$ may be not defined, the equilibrium strategies of $\mathbf{s}$ cannot be satisfactorily defined on the basis of the sequence $x_{\mathbf{s}}^{*}\left(t, \mathbf{b}_{i}\right)$. To be exact, this happens at every time point the optimal action of $\mathbf{s}$ is not $\operatorname{offer}(\cdot)$, but is $\operatorname{match}(\cdot)$. To cope with this problem we resort to the notion of equivalent value $e_{\mathbf{s}}\left(\sigma, t, \mathbf{b}_{i}\right)$ of an action $\sigma$ performed by $\mathbf{s}$ at $t$ in $\Gamma\left(t, \mathbf{b}_{i}, \mathbf{s}\right)$ : we define it as the value such that $U_{\mathbf{s}}\left(e_{\mathbf{s}}\left(\sigma, t, \mathbf{b}_{i}\right), t\right)=E U_{\mathbf{s}}\left(\sigma, t, \mathbf{b}_{i}\right)$ where $E U_{\mathbf{s}}$ is the utility expected by $\mathbf{s}$ from making $\sigma$ at $t$ in $\Gamma\left(t, \mathbf{b}_{i}, \mathbf{s}\right)$. We denote by $e_{\mathbf{s}}^{*}\left(t, \mathbf{b}_{i}\right)$ the optimal equivalent value of $\mathbf{s}$ at $t$ in $\Gamma\left(t, \mathbf{b}_{i}, \mathbf{s}\right)$, i.e., the equivalent value of the optimal action.

As we shall show below, the equilibrium strategies of $\mathbf{s}$ can be satisfactorily defined on the basis of $x_{\mathbf{s}}^{*}\left(t, \mathbf{b}_{i}\right)$ and $e_{\mathbf{s}}^{*}\left(t, \mathbf{b}_{i}\right)$ : if $\mathbf{s}$ receives an offer $x \geq\left(e_{\mathbf{s}}^{*}\left(t, \mathbf{b}_{i}\right)\right)_{\leftarrow \mathbf{s}}$ at $t$, then she accepts it, otherwise she rejects it to offer $x_{\mathbf{s}}^{*}\left(t, \mathbf{b}_{i}\right)$, if $x_{\mathbf{s}}^{*}\left(t, \mathbf{b}_{i}\right)$ is defined, and to choose $\operatorname{match}\left(\mathbf{b}_{j}\right)$ with $j \neq i$, if $x_{\mathbf{s}}^{*}\left(t, \mathbf{b}_{i}\right)$ is not defined. This is to say that $\mathbf{s}$ would accept any offer that gives her a utility equal to or larger than the utility she expects from making her optimal action between making an offer and matching a different opponent. The value $x_{\mathbf{s}}^{*}\left(t, \mathbf{b}_{i}\right)$ is such that it will be accepted by $\mathbf{b}_{i}$ at $t+1$. When $\sigma=\operatorname{offer}(x)$ and $x$ is an offer that will be accepted, $E U_{\mathbf{s}}\left(\sigma, t, \mathbf{b}_{i}\right)$ is exactly $U_{\mathbf{s}}(x, t)$ and then $e_{\mathbf{s}}\left(\sigma, t, \mathbf{b}_{i}\right)=x$ independently of $i$ and $t$. When $\sigma=\operatorname{match}\left(\mathbf{b}_{i}\right)$, the formula of $E U_{\mathbf{s}}\left(\sigma, t, \mathbf{b}_{j}\right)$ with $i \neq j$ is similar to the one reported in Section 4.1. Indeed, at $t+d$ two subgames are possible with probability .5 each: the subgame $\Gamma\left(t+d, \mathbf{b}_{i}, \mathbf{b}_{i}\right)$ where $\mathbf{b}_{i}$ acts at $t+d$ and the subgame $\Gamma\left(t+d, \mathbf{b}_{i}, \mathbf{s}\right)$ where $\mathbf{s}$ act at such time point. Therefore, $E U_{\mathrm{s}}$ is obtained firstly computing the average between the utility gained by $\mathbf{s}$

\footnotetext{
${ }^{7}$ We shall show in the next section that equilibrium strategies that prescribe $\mathbf{b}_{i}$ to choose matchable(s) lead to non-reasonable agents' behaviors and we discuss how these non-reasonable equilibria can be removed.
} 
from $\Gamma\left(t+d, \mathbf{b}_{i}, \mathbf{b}_{i}\right)$ and the one from $\Gamma\left(t+d, \mathbf{b}_{i}, \mathbf{s}\right)$ and subsequently discounting it for $d$ time points with respect to $\delta_{\mathbf{s}}$. Precisely, $\mathbf{s}$ expects $U_{\mathbf{s}}\left(x_{\mathbf{b}_{i}}^{*}(t+d), t+d\right)$ from $\Gamma\left(t+d, \mathbf{b}_{i}, \mathbf{b}_{i}\right)$ - this is because, if $\mathbf{b}_{i}$ opens the negotiation, she will make an offer that will be accepted by $\mathbf{s}$ - and expects $U_{\mathbf{s}}\left(e_{\mathbf{s}}^{*}\left(t+d, \mathbf{b}_{i}\right), t+d\right)$ from $\Gamma\left(t+d, \mathbf{b}_{i}, \mathbf{s}\right)$ - this is because, if $\mathbf{s}$ opens the negotiation, she could make match $(\cdot)$ or an offer. Therefore, the equivalent value $e_{\mathbf{s}}$ of making $\operatorname{match}\left(\mathbf{b}_{i}\right)$ at $t$ is $\left(\frac{1}{2} x_{\mathbf{b}_{i}}^{*}(t+d)+\frac{1}{2} e_{\mathbf{s}}^{*}\left(t+d, \mathbf{b}_{i}\right)\right)_{\leftarrow d[\mathbf{s}]}$.

We now apply the backward induction method from time point $t=\bar{T}$ back. For the sake of presentation, we divide the backward induction construction in several parts with respect to both time $t$ and the buyer with whom $\mathbf{s}$ is currently negotiating, and we analyze them singularly. We report such a division in Table 2. This division has the property that agents' optimal actions in all the time points belonging to the same part of construction are given by the same choice rules, whereas agents' optimal actions in time points belonging to different parts of the construction are given by different choice rules.

Consider the subgames $\Gamma\left(t, \mathbf{b}_{2}, \mathbf{b}_{2}\right)$ and $\Gamma\left(t, \mathbf{b}_{2}, \mathbf{s}\right)$ at $T_{\mathbf{b}_{1}}-d \leq t \leq \bar{T}-1$. At these time points $\mathbf{s}$ cannot choose match $\left(\mathbf{b}_{1}\right)$, since $\mathbf{b}_{1}$ will never choose matchable. Therefore, agents' strategies are those provided in Section 2.2. The values of $x_{\mathbf{b}_{2}}^{*}(t)$ and $x_{\mathbf{s}}^{*}\left(t, \mathbf{b}_{2}\right)$ can be computed as follows:

$$
\begin{cases}t=\bar{T}-1 & \begin{cases}x_{\mathbf{b}_{2}}^{*}(t) & =R P_{\mathbf{s}} \\
x_{\mathbf{s}}^{*}\left(t, \mathbf{b}_{2}\right) & =R P_{\mathbf{b}} \\
e_{\mathbf{s}}^{*}\left(t, \mathbf{b}_{2}\right) & =x_{\mathbf{s}}^{*}\left(t, \mathbf{b}_{2}\right)\end{cases} \\
T_{\mathbf{b}_{1}}-d \leq t<\bar{T}-1 & \left\{\begin{array}{ll}
x_{\mathbf{b}_{2}}^{*}(t) & =\left(x_{\mathbf{s}}^{*}\left(t+1, \mathbf{b}_{2}\right)\right)_{\leftarrow \mathbf{s}} \\
x_{\mathbf{s}}^{*}\left(t, \mathbf{b}_{2}\right) & =\left(x_{\mathbf{b}_{2}}^{*}(t+1)\right)_{\leftarrow} \mathbf{b}_{2} \\
e_{\mathbf{s}}^{*}\left(t, \mathbf{b}_{2}\right) & =x_{\mathbf{s}}^{*}\left(t, \mathbf{b}_{2}\right)
\end{array} .\right.\end{cases}
$$

The optimal strategies of $\mathbf{b}_{2}$ and $\mathbf{s}$ can be easily provided on the basis of the optimal offers as follows:

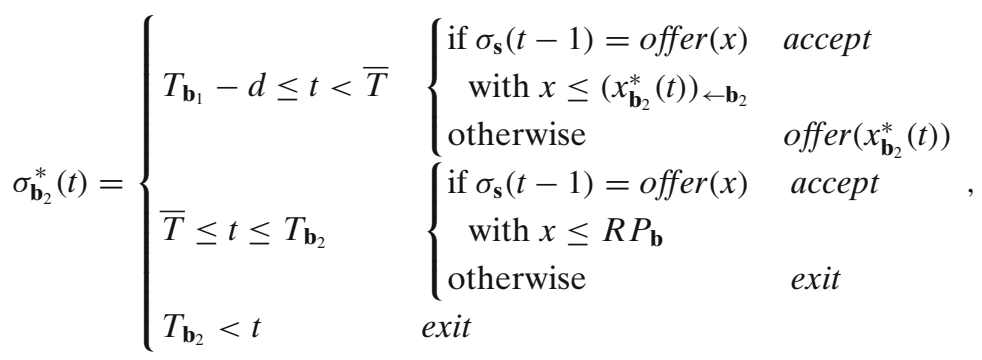

Table 2 Intervals of time in which we divide the backward induction construction

\begin{tabular}{lll}
\hline Strategies $\sigma_{\mathbf{b}_{1}}^{*}(t), \sigma_{\mathbf{s}}^{*}\left(t, \mathbf{b}_{1}\right)$ based on $x_{\mathbf{b}_{1}}^{*}(t), e_{\mathbf{s}}^{*}\left(t, \mathbf{b}_{1}\right)$ & \\
{$\left[0, T_{\mathbf{b}_{1}}-d\right] \quad\left[T_{\mathbf{b}_{1}}-d, \min \left\{\bar{T}-d, T_{\mathbf{b}_{1}}-1\right\}\right]$} & {$\left[\min \left\{\bar{T}-d, T_{\mathbf{b}_{1}}-1\right\}, \max \left\{\bar{T}-d, T_{\mathbf{b}_{1}}-1\right\}\right]$} \\
Strategies $\sigma_{\mathbf{b}_{2}}^{*}(t), \sigma_{\mathbf{s}}^{*}\left(t, \mathbf{b}_{2}\right)$ based on $x_{\mathbf{b}_{2}}^{*}(t), e_{\mathbf{s}}^{*}\left(t, \mathbf{b}_{2}\right)$ & \\
{$\left[0, T_{\mathbf{b}_{1}}-d\right]$} & {$\left[T_{\mathbf{b}_{1}}-d, \bar{T}-1\right]$} & \\
\hline
\end{tabular}




$$
\sigma_{\mathbf{s}}^{*}\left(t, \mathbf{b}_{2}\right)=\left\{\begin{array}{lll}
T_{\mathbf{b}_{1}}-d \leq t<\bar{T} & \left\{\begin{array}{ll}
\text { if } \sigma_{\mathbf{b}_{2}}(t-1)=\text { offer }(x) & \text { accept } \\
\text { with } x \geq\left(x_{\mathbf{s}}^{*}\left(t, \mathbf{b}_{2}\right)\right)_{\leftarrow \mathbf{s}} & \\
\text { otherwise } & \text { offer }\left(x_{\mathbf{s}}^{*}\left(t, \mathbf{b}_{2}\right)\right) \\
\bar{T} \leq t \leq T_{\mathbf{s}} & \begin{cases}\text { if } \sigma_{\mathbf{b}_{2}}(t-1)=\text { offer }(x) & \text { accept } \\
\text { with } x \geq R P_{\mathbf{s}} & \text { exit } \\
\text { otherwise } & \text { exit }\end{cases}
\end{array} .\right.
\end{array}\right.
$$

Consider the subgames $\Gamma\left(t, \mathbf{b}_{1}, \mathbf{b}_{1}\right)$ and $\Gamma\left(t, \mathbf{b}_{1}, \mathbf{s}\right)$ at $\min \left\{\bar{T}-d, T_{\mathbf{b}_{1}}-1\right\} \leq t \leq$ $\max \left\{\bar{T}-d, T_{\mathbf{b}_{1}}-1\right\}$. Two situations can be: in the first one $\bar{T}-d \leq T_{\mathbf{b}_{1}}$ and in the second one $T_{\mathbf{b}_{1}}<\bar{T}-d$. In the first situation the optimal strategies of $\mathbf{b}_{1}$ and $\mathbf{s}$ can be computed as above, since $\mathbf{b}_{2}$ will never make matchable(s) at $t \geq \bar{T}-d$ and then $\mathbf{s}$ cannot choose match $\left(\mathbf{b}_{2}\right)$. In the second situation instead, the backward induction construction is strongly different. Indeed, in $\Gamma\left(T_{\mathbf{b}_{1}}-1, \mathbf{b}_{1}, \mathbf{b}_{1}\right) \mathbf{s}$ is not obliged to accept her reservation price at $t=T_{\mathbf{b}_{1}}$ (as instead it happens when a unique buyer is present), but she can make match $\left(\mathbf{b}_{2}\right)$. The equivalent value of $\operatorname{match}\left(\mathbf{b}_{2}\right)$ is $\left(\frac{1}{2} x_{\mathbf{b}_{2}}^{*}(t+d)+\frac{1}{2} x_{\mathbf{s}}^{*}\left(t+d, \mathbf{b}_{2}\right)\right)_{\leftarrow d[\mathbf{s}]}$ and is obviously larger than $R P_{\mathbf{s}}$. Therefore, the offer that $\mathbf{s}$ would accept at $t=T_{\mathbf{b}_{1}}$ is the offer that gives her a utility equal to or larger than making match $\left(\mathbf{b}_{2}\right)$, namely, any offer $x$ such that $x \geq\left(\frac{1}{2} x_{\mathbf{b}_{2}}^{*}(t+d)+\frac{1}{2} x_{\mathbf{s}}^{*}\left(t+d, \mathbf{b}_{2}\right)\right)_{\leftarrow(d+1)[\mathbf{s}]}$. The availability of the outside option for $\mathbf{s}$ makes the position of $\mathbf{s}$ at $t=T_{\mathbf{b}_{1}}$ stronger than her position in the situation $\mathbf{s}$ has not any outside option. As such, the revenue that $\mathbf{s}$ expects to gain increases with respect to the purely bilateral situation where there is no competition among buyers. In addition, notice that for any $T_{\mathbf{b}_{1}}<t<\bar{T}-d$ the optimal action of $\mathbf{s}$ in $\Gamma\left(t, \mathbf{b}_{1}, \mathbf{s}\right)$ is to choose match $\left(\mathbf{b}_{2}\right)$. The values of $x_{\mathbf{b}_{1}}^{*}(t), x_{\mathbf{s}}^{*}\left(t, \mathbf{b}_{1}\right)$, and $x_{\mathbf{s}}^{*}\left(t, \mathbf{b}_{1}\right)$ can be computed as follows:

$$
\begin{cases}t=T_{\mathbf{b}_{1}}-1 \text { and } \bar{T}-d \leq T_{\mathbf{b}_{1}}-1 & \begin{cases}x_{\mathbf{b}_{1}}^{*}(t) & =R P_{\mathbf{s}} \\
x_{\mathbf{s}}^{*}\left(t, \mathbf{b}_{1}\right) & =R P_{\mathbf{b}} \\
e_{\mathbf{s}}^{*}\left(t, \mathbf{b}_{1}\right) & =R P_{\mathbf{b}}\end{cases} \\
\bar{T}-d \leq t<T_{\mathbf{b}_{1}}-1 & \begin{cases}x_{\mathbf{b}_{1}}^{*}(t) & \left.=\left(x_{\mathbf{s}}^{*}\left(t+1, \mathbf{b}_{1}\right)\right)_{\leftarrow \mathbf{s}}\right) \\
x_{\mathbf{s}}^{*}\left(t, \mathbf{b}_{1}\right) & \left.=\left(x_{\mathbf{b}_{1}}^{*}(t+1)\right)_{\leftarrow \mathbf{b}_{1}}\right) \\
e_{\mathbf{s}}^{*}\left(t, \mathbf{b}_{1}\right) & =x_{\mathbf{s}}^{*}\left(t, \mathbf{b}_{1}\right)\end{cases} \\
T_{\mathbf{b}_{1} \leq t<\bar{T}-d} \leq \begin{array}{ll}
x_{\mathbf{b}_{1}}^{*}(t) & \text { is not defined } \\
x_{\mathbf{s}}^{*}\left(t, \mathbf{b}_{1}\right) & \text { is not defined } \\
e_{\mathbf{s}}^{*}\left(t, \mathbf{b}_{1}\right) & =\left(\frac{1}{2} x_{\mathbf{b}_{2}}^{*}(t+d)+\frac{1}{2} x_{\mathbf{s}}^{*}\left(t+d, \mathbf{b}_{2}\right)\right)_{\leftarrow d[\mathbf{s}]}
\end{array} & \begin{cases}x_{\mathbf{b}_{1}}^{*}(t) & =\left(\frac{1}{2} x_{\mathbf{b}_{2}}^{*}(t+d+1)\right. \\
t=T_{\mathbf{b}_{1}}-1 \text { and } T_{\mathbf{b}_{1}}-1<T-d & \left.+\frac{1}{2} x_{\mathbf{s}}^{*}\left(t+d+1, \mathbf{b}_{2}\right)\right)_{\leftarrow(d+1)[\mathbf{s}]} \\
x_{\mathbf{s}}^{*}\left(t, \mathbf{b}_{1}\right) & =R P_{\mathbf{b}} \\
e_{\mathbf{s}}^{*}\left(t, \mathbf{b}_{1}\right) & =R P_{\mathbf{b}}\end{cases} \end{cases}
$$


The optimal strategies of $\mathbf{b}_{1}$ and $\mathbf{s}$ can be easily defined on the basis of $x_{\mathbf{b}_{1}}^{*}(t)$, $x_{\mathbf{s}}^{*}\left(t, \mathbf{b}_{1}\right)$, and $x_{\mathbf{s}}^{*}\left(t, \mathbf{b}_{1}\right)$. They are:

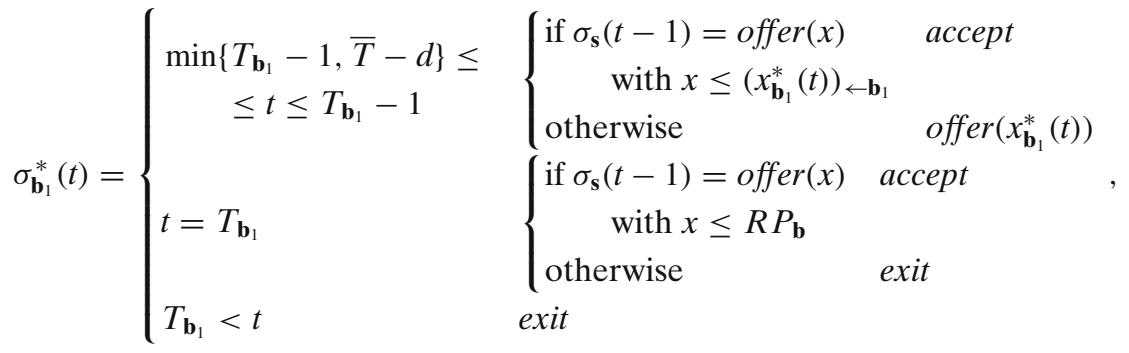

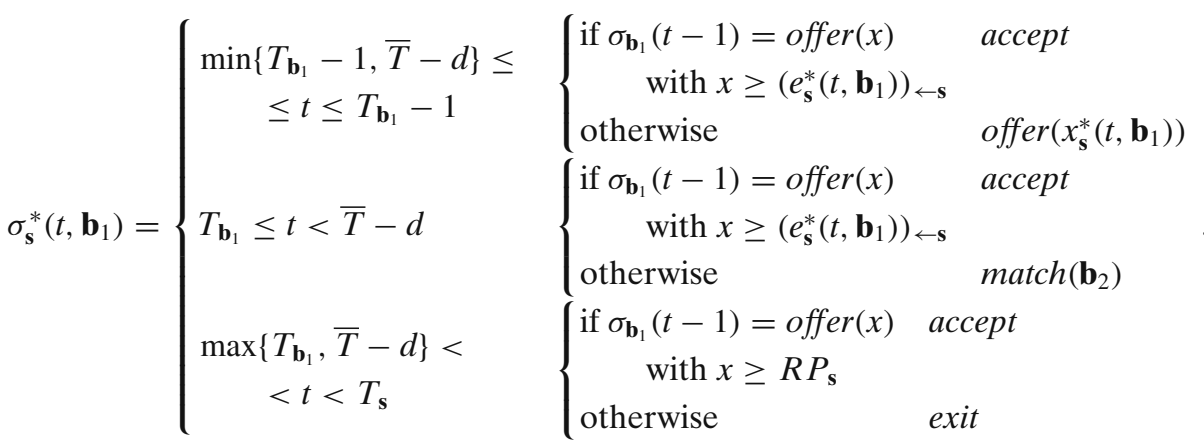

Consider the subgames $\Gamma\left(t, \mathbf{b}_{1}, \mathbf{b}_{1}\right)$ and $\Gamma\left(t, \mathbf{b}_{1}, \mathbf{s}\right)$ at $T_{\mathbf{b}_{1}}-d \leq t<\min \{\bar{T}-$ $\left.d, T_{\mathbf{b}_{1}}-1\right\}$. In this interval of time, $\mathbf{s}$ can choose match $\left(\mathbf{b}_{2}\right)$, since $\mathbf{b}_{2}$ chooses matchable(s). The optimal offer of $\mathbf{b}_{1}$ at $t$ is the lowest offer that $\mathbf{s}$ would accept at $t+1$, namely, $\left(e^{*}\left(t+1, \mathbf{b}_{1}\right)\right)_{\leftarrow \mathbf{s}}$. The proof is trivial when $\mathbf{s}$ 's optimal action at $t+1$ is to make an offer. Instead, when s's optimal action at $t+1$ is to make match(.), the proof is very involved and we discuss it in Appendix B. This proof represents the main result on bargaining with outside option, since allows one to consider a very narrow set of possible actions in the search for the optimal agents' strategies, drastically reducing thus the computational complexity of finding an equilibrium. The optimal action of $\mathbf{s}$ at $t$ is the action between $\operatorname{offer}\left(x_{\mathbf{b}_{1}}^{*}(t+1)\right)_{\leftarrow \mathbf{s}}$ and $\operatorname{match}\left(\mathbf{b}_{2}\right)$ with the greatest equivalent value, i.e., the action that maximizes the expected utility of $\mathbf{s}$. Therefore, the optimal action of $\mathbf{s}$ can be found at any time point through a maximization between only these two options. Notice that in this interval of time $\sigma_{\mathbf{b}_{1}}^{*}(t)$ and $\sigma_{\mathbf{s}}^{*}\left(t, \mathbf{b}_{1}\right)$ depend on $\sigma_{\mathbf{b}_{2}}^{*}(t+d)$ and $\sigma_{\mathbf{s}}^{*}\left(t+d, \mathbf{b}_{2}\right)$, but $\sigma_{\mathbf{b}_{2}}^{*}(t)$ and $\sigma_{\mathbf{s}}^{*}\left(t, \mathbf{b}_{2}\right)$ do not depend on $\sigma_{\mathbf{b}_{1}}^{*}(t+d)$ and $\sigma_{\mathbf{s}}^{*}\left(t+d, \mathbf{b}_{1}\right)$.

The equilibrium strategies in all subgames at $0 \leq t<T_{\mathbf{b}_{1}}-d$ can be found similarly. Here $\sigma_{\mathbf{b}_{1}}^{*}(t)$ and $\sigma_{\mathbf{b}_{2}}^{*}(t)$ can be found in closed form (since the corresponding optimal offers can be found in closed form), whereas the computation of $\sigma_{\mathbf{s}}^{*}\left(t, \mathbf{b}_{1}\right)$ and $\sigma_{\mathbf{s}}^{*}\left(t, \mathbf{b}_{2}\right)$ requires at each time point a maximization between two possible options: offer $\left(\left(x^{*}(t+1) \leftarrow_{\leftarrow \mathbf{b}_{1}}\right)\right.$ and $\operatorname{match}\left(\mathbf{b}_{2}\right)$ for the determination of $\sigma_{\mathbf{s}}^{*}\left(t, \mathbf{b}_{1}\right)$ and $\operatorname{offer}\left(\left(x^{*}(t+1)\right)_{\leftarrow \mathbf{b}_{2}}\right)$ and match $\left(\mathbf{b}_{1}\right)$ for the determination of $\sigma_{\mathbf{s}}^{*}\left(t, \mathbf{b}_{2}\right)$. Notice that in this interval of time $\sigma_{\mathbf{b}_{1}}^{*}(t)$ and $\sigma_{\mathbf{s}}^{*}\left(t, \mathbf{b}_{1}\right)$ depend on $\sigma_{\mathbf{b}_{2}}^{*}(t+d)$ and $\sigma_{\mathbf{s}}^{*}\left(t+d, \mathbf{b}_{2}\right)$, and vice versa. We consider now the utility $\mathbf{b}_{i}$ expects from starting a negotiation at $t+d$ when $t<\min \left\{T_{\mathbf{b}_{i}}, \bar{T}\right\}-d$. It is equal to or larger than $U_{\mathbf{b}_{i}}\left(\frac{1}{2} x_{\mathbf{b}_{i}}^{*}(t+d), d\right)$. Indeed, if she opens the negotiation, she will offer $x_{\mathbf{b}_{i}}^{*}(t+d)$ and $\mathbf{s}$ will accept such offer. Since 
it holds $R P_{\mathbf{b}_{1}}=R P_{\mathbf{b}_{2}}=R P_{\mathbf{b}}>R P_{\mathbf{s}}$, all the values in the sequences $x_{\mathbf{b}_{1}}^{*}(t)$ and $x_{\mathbf{b}_{2}}^{*}(t)$ are strictly lower than $R P_{\mathbf{b}}$, and therefore each $\mathbf{b}_{i}$ expects a strictly positive utility at any $t<\min \left\{T_{\mathbf{b}_{i}}, \bar{T}\right\}-d$. The optimal offers and equivalent values are:

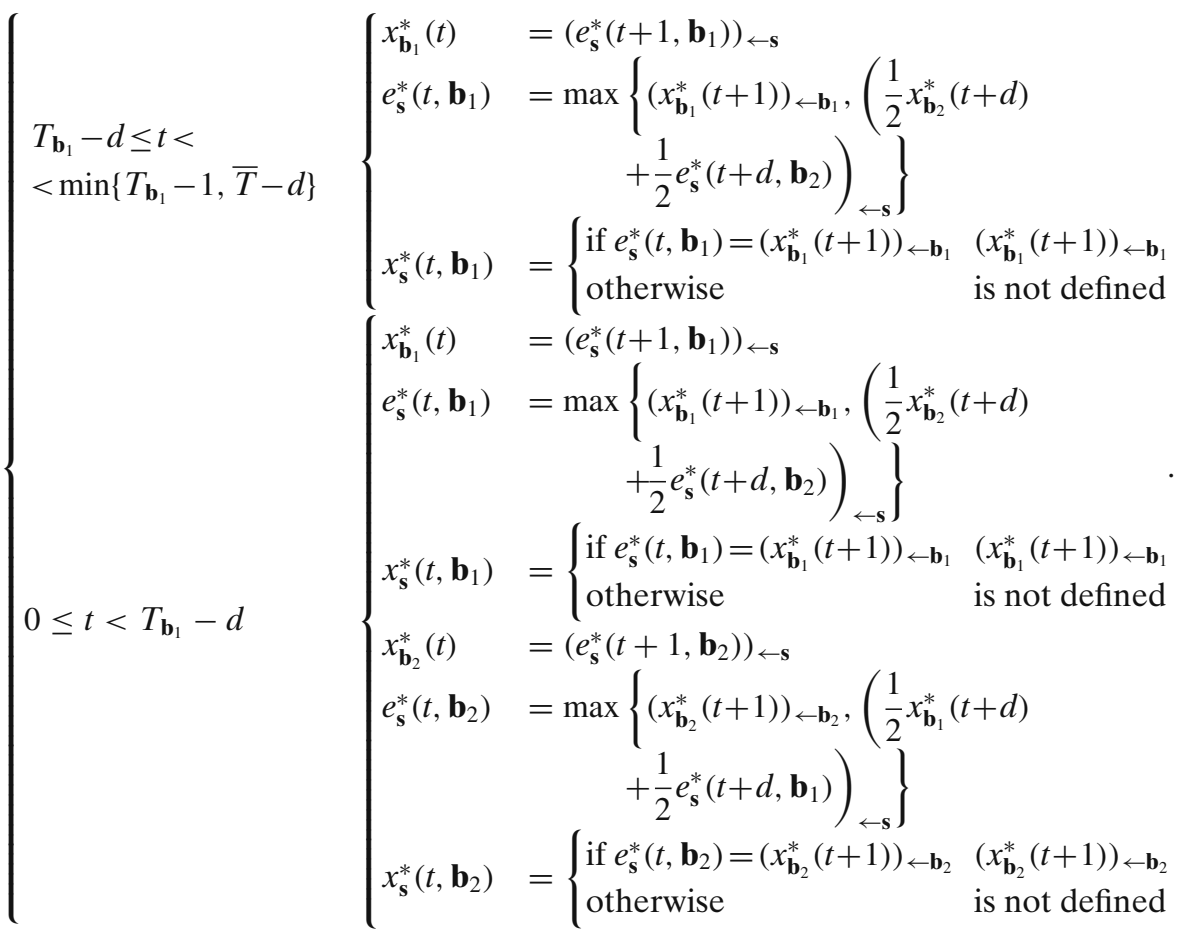

The equilibrium strategies can be defined on the basis of the above sequences as follows:

$$
\begin{aligned}
& \sigma_{\mathbf{b}_{1}}^{*}(t)=\left\{\begin{array} { c l } 
{ t = 0 } & { \text { offer } ( x _ { \mathbf { b } _ { 1 } } ^ { * } ( t ) ) } \\
{ 0 < t < \operatorname { m i n } } \\
{ \{ T _ { \mathbf { b } _ { 1 } } - 1 , } \\
{ \overline { T } - d \} }
\end{array} \quad \left\{\begin{array}{ll}
\text { if } \sigma_{\mathbf{s}}(t-1)=\text { offer }(x) \text { with } x \leq\left(x_{\mathbf{b}_{1}}^{*}(t)\right)_{\leftarrow} \mathbf{b}_{1} & \text { accept } \\
\text { otherwise } & \text { offer }\left(x_{\mathbf{b}_{1}}^{*}(t)\right)
\end{array},\right.\right.
\end{aligned}
$$

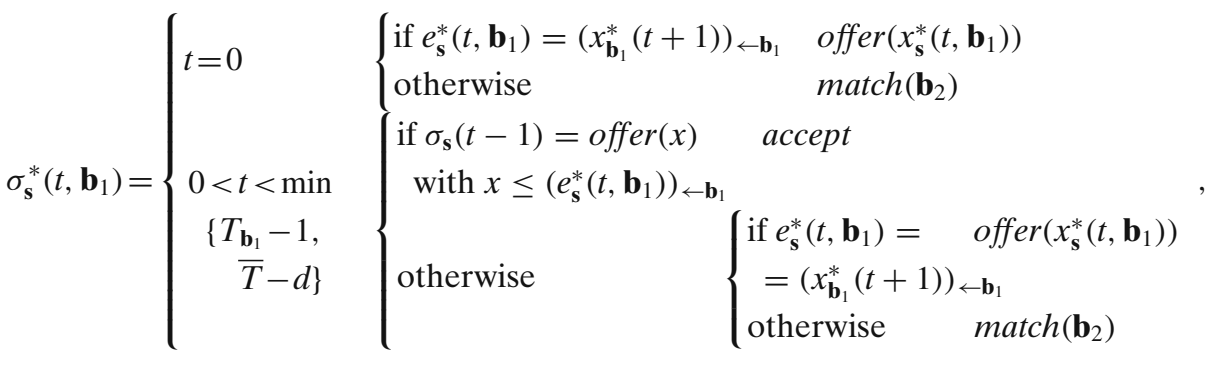

$$
\begin{aligned}
& \sigma_{\mathbf{b}_{2}}^{*}(t)=\left\{\begin{array} { l l } 
{ t = 0 } & { \text { offer } ( x _ { \mathbf { b } _ { 2 } } ^ { * } ( t ) ) } \\
{ 0 < t < } \\
{ < T _ { \mathbf { b } _ { 1 } } - d }
\end{array} \quad \left\{\begin{array}{ll}
\text { if } \sigma_{\mathbf{s}}(t-1)=\text { offer }(x) \text { with } x \leq\left(x_{\mathbf{b}_{2}}^{*}(t)\right)_{\leftarrow \mathbf{b}_{2}} & \text { accept } \\
\text { otherwise } & \text { offer }\left(x_{\mathbf{b}_{2}}^{*}(t)\right)
\end{array},\right.\right.
\end{aligned}
$$




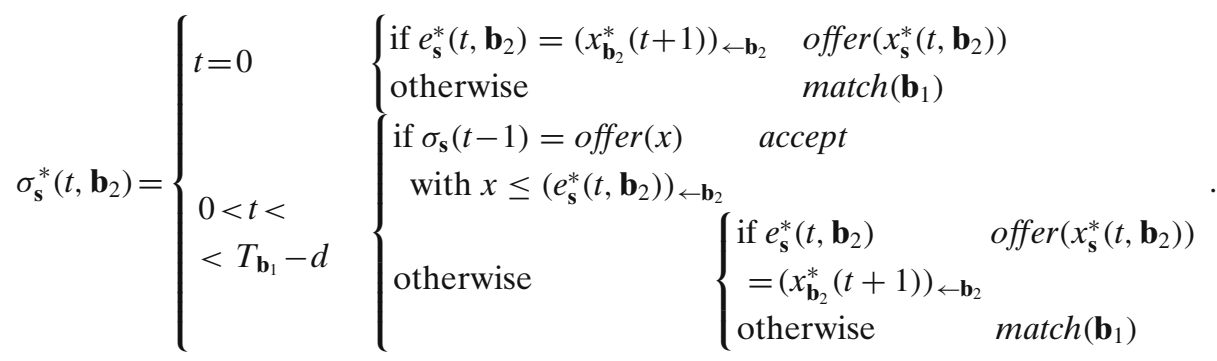

Once we have found the equilibrium strategies from $t=0$ onwards, we can easily find the opponent to whom s can be optimally matched at $t=-d$. More specifically, it is the buyer $\mathbf{b}_{i}$ such that $x_{\mathbf{b}_{i}}^{*}(0)+e_{\mathbf{s}}^{*}\left(0, \mathbf{b}_{i}\right)$ is maximum. The equilibrium strategies can be found by calculating the sequences: $x_{\mathbf{b}_{1}}^{*}(t), x_{\mathbf{b}_{2}}^{*}(t), x_{\mathbf{s}}^{*}\left(t, \mathbf{b}_{1}\right), x_{\mathbf{s}}^{*}\left(t, \mathbf{b}_{2}\right), e_{\mathbf{s}}^{*}\left(t, \mathbf{b}_{1}\right)$, $e_{\mathbf{s}}^{*}\left(t, \mathbf{b}_{2}\right)$. Therefore the computational complexity required to produce the above equilibrium strategies is $O(\bar{T})$ with single-issue negotiations and $O(l \cdot \bar{T})$ with $l$ issue negotiations.

We summarize the equilibrium strategies in Table 3 . We can state now the following theorem (it holds by construction, being the above strategies produced by backward induction).

Theorem 5.1 The strategies provided in Table 3 constitute a subgame perfect equilibrium.

In Fig. 4 we report the backward induction constructions of the games $\Gamma\left(0, \mathbf{b}_{1}, \mathbf{b}_{1}\right)$, $\Gamma\left(0, \mathbf{b}_{1}, \mathbf{s}\right), \Gamma\left(0, \mathbf{b}_{2}, \mathbf{b}_{2}\right)$, and $\Gamma\left(0, \mathbf{b}_{2}, \mathbf{s}\right)$ with $R P_{\mathbf{b}_{1}}=R P_{\mathbf{b}_{2}}=1, \delta_{\mathbf{b}_{1}}=\delta_{\mathbf{b}_{2}}=.95, T_{\mathbf{b}_{1}}=$ $9, T_{\mathbf{b}_{2}}=11, R P_{\mathbf{s}}=0, \delta_{\mathbf{s}}=.95, T_{\mathbf{s}}=12$, and $d=1$. We report the agents' optimal offers at the time points where they are defined and the optimal equivalent values where the optimal offers are not defined; the dashed line denotes the construction when action match $(\cdot)$ is not available. For the sake of clarity, we emphasize the

Table 3 Agents' optimal actions in the presence of two buyers with the same reservation prices and arrival time

\begin{tabular}{|c|c|c|c|c|}
\hline Situation & Stage & Agents & Time points & Optimal actions \\
\hline \multirow[t]{3}{*}{$\begin{array}{c}\mathbf{b}_{i}(\mathbf{s}) \text { is not } \\
\text { matched }\end{array}$} & 1 & $\mathbf{b}_{i}$ & Any & $\begin{array}{l}\text { matchable }(\mathbf{s}) \text { if } t<\min \left\{T_{\mathbf{b}_{i}}, \bar{T}\right\}-d, \\
\text { nonmatchable otherwise }\end{array}$ \\
\hline & & $\mathbf{b}_{i}$ & Any & $\begin{array}{l}\text { wait if } t<\min \left\{T_{\mathbf{b}_{i}}, \bar{T}\right\}-d \\
\text { exit otherwise }\end{array}$ \\
\hline & 2 & $\mathbf{s}$ & Any & $\begin{array}{l}\operatorname{match}\left(\mathbf{b}_{i}\right) \text { with } i= \\
\quad \arg \max _{i}\left\{x_{\mathbf{b}_{i}}^{*}(t+d)+e_{\mathbf{s}}^{*}\left(t+d, \mathbf{b}_{i}\right)\right\} \\
\quad \text { if } t<\bar{T}-d \text {, exit otherwise }\end{array}$ \\
\hline $\begin{array}{l}\mathbf{b}_{i}(\mathbf{s}) \text { is } \\
\text { negotiating }\end{array}$ & 2 & $\begin{array}{l}\mathbf{b}_{i} \\
\mathbf{s}\end{array}$ & $\begin{array}{l}\text { Alternately } \\
\text { Alternately }\end{array}$ & $\begin{array}{l}\sigma_{\mathbf{b}_{i}}^{*}(t) \text { defined in Section } 5.1 \\
\sigma_{\mathbf{s}}^{*}\left(t, \mathbf{b}_{i}\right) \text { defined in Section } 5.1\end{array}$ \\
\hline
\end{tabular}



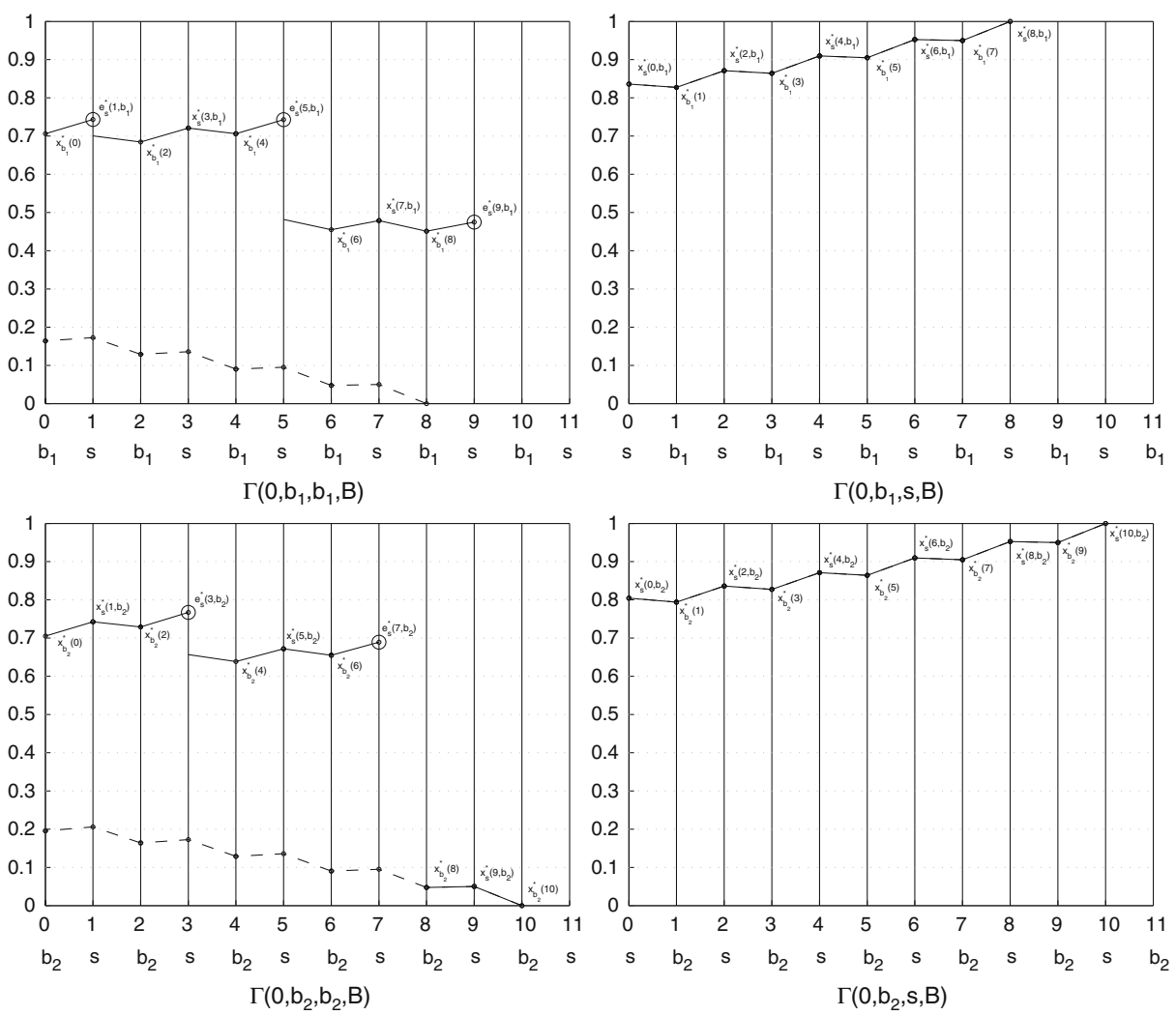

Fig. 4 Backward induction constructions of the games $\Gamma\left(0, \mathbf{b}_{1}, \mathbf{s}\right)$ and $\Gamma\left(0, \mathbf{b}_{2}, \mathbf{s}\right)$ with $R P_{\mathbf{b}_{1}}=$ $R P_{\mathbf{b}_{2}}=1, \delta_{\mathbf{b}_{1}}=\delta_{\mathbf{b}_{2}}=.95, T_{\mathbf{b}_{1}}=9, T_{\mathbf{b}_{2}}=11, R P_{\mathbf{s}}=0, \delta_{\mathbf{s}}=.95, T_{\mathbf{s}}=12$, and $d=1$

optimal equivalent values with a large circle: at such time points $\mathbf{s}$ chooses $\operatorname{match}(\cdot) .{ }^{8}$ The backward induction constructions in the games $\Gamma\left(0, \mathbf{b}_{1}, \mathbf{s}\right)$ and $\Gamma\left(0, \mathbf{b}_{2}, \mathbf{s}\right)$ in this setting are the same as the ones in the corresponding games in which action match( $\cdot)$ is not available (the construction in $\Gamma\left(0, \mathbf{b}_{2}, \mathbf{s}\right)$ is shown in Fig. 1 , the construction in $\Gamma\left(0, \mathbf{s}, \mathbf{b}_{1}\right)$ is the same one as $\Gamma\left(0, \mathbf{b}_{1}, \mathbf{s}\right)$, but it is translated two time points to the left $)$. The optimal offers in $\Gamma\left(t, \mathbf{b}_{2}, \mathbf{b}_{2}\right)$ for $8 \leq t \leq 11$ are the same as the corresponding game in which the action match $(\cdot)$ is not available. This is because such time points are beyond $T_{\mathbf{b}_{1}}-d$ and then the strategies in such subgames are not affected by the presence of $\mathbf{b}_{1}$. The optimal offers in $\Gamma\left(0, \mathbf{b}_{2}, \mathbf{b}_{2}\right)$ are $x_{\mathbf{b}_{2}}^{*}(10)=0, x_{\mathbf{s}}^{*}\left(9, \mathbf{b}_{2}\right)=.05$, and $x_{\mathbf{b}_{2}}^{*}(8)=.0475$, whereas the optimal offers in $\Gamma\left(0, \mathbf{b}_{2}, \mathbf{s}\right)$ are $x_{\mathbf{s}}^{*}\left(10, \mathbf{b}_{2}\right)=1, x_{\mathbf{b}_{2}}^{*}(9)=.95$, and $x_{\mathbf{s}}^{*}\left(8, \mathbf{b}_{2}\right)=.9525$. Consider time point $t=9$ in $\Gamma\left(9, \mathbf{b}_{1}, \mathbf{s}\right)$. In the corresponding game wherein $\operatorname{match}\left(\mathbf{b}_{2}\right)$ is not available,

\footnotetext{
${ }^{8}$ Notice that the equivalents $e_{\mathbf{s}}^{*}\left(t, \mathbf{b}_{i}\right)$ of choosing match $(\cdot)$ at $t$ are not on the isoutility curves of $x_{\mathbf{b}_{i}}^{*}(t+1) \mathrm{s}$, but they are higher. Indeed, $\mathbf{s}$ can gain more with respect to the setting where she cannot exploit any outside option.
} 
s should accept any offer equal to or larger than her reservation price, namely, any $x \geq 0$. Here, $\mathbf{s}$ chooses match $\left(\mathbf{b}_{2}\right)$ and then she would accept any offer equal to or larger than $\left(e^{*}\left(9, \mathbf{b}_{1}\right)\right)_{\leftarrow \mathbf{s}}$ where $e^{*}\left(9, \mathbf{b}_{1}\right)$ is the equivalent value of choosing $\operatorname{match}\left(\mathbf{b}_{2}\right)$, more precisely, it is $\left(\frac{1}{2} x_{\mathbf{b}_{2}}^{*}(10)+\frac{1}{2} x_{\mathbf{s}}^{*}\left(10, \mathbf{b}_{2}\right)\right)_{\leftarrow \mathbf{s}}=.475$. The value of $x_{\mathbf{b}_{1}}^{*}(8)$ is $\simeq .45$. The value of $x_{\mathbf{s}}^{*}\left(8, \mathbf{b}_{1}\right)$ in $\Gamma\left(8, \mathbf{b}_{1}, \mathbf{s}\right)$ is $R P_{\mathbf{b}}=1$. Consider now time point $t=7$ in $\Gamma\left(7, \mathbf{b}_{2}, \mathbf{s}\right)$. In the corresponding game wherein match $\left(\mathbf{b}_{1}\right)$ is not available, $\mathbf{s}$ would offer $\simeq .095$. Here, she chooses $\operatorname{match}\left(\mathbf{b}_{1}\right)$ whose equivalent value is $\simeq 0.69$. The construction continues to time point $t=0$.

Notice that the gain of $\mathbf{s}$ significantly increases with respect to the situation in which there is no competition between buyers and therefore can effectively take advantage from the competition between $\mathbf{b}_{1}$ and $\mathbf{b}_{2}$. Moreover, if any buyer except the one with the latest deadline acts at the time point before her deadline, she cannot impose $\mathbf{s}$ to accept her reservation price since $\mathbf{s}$ can leave the negotiation and start a new one. Therefore, the position of the agent that act at the time point before the deadline gets weaker and the position of the agent with the longer deadline gets stronger with respect to the classic alternating-offers protocol.

\subsection{Addressing equilibrium uniqueness}

In this section, at first we show that multiple equilibria can be when agents can exploit outside option and subsequently we show that the introduction of costs for agents during the negotiation can lead to equilibrium uniqueness.

We show that the protocol can admit multiple equilibria. Consider, for instance, the subgame $\Gamma\left(8, \mathbf{b}_{2}, \mathbf{s}\right)$ depicted in Fig. 5 for $t \geq 8$ : there are two buyers $\mathbf{b}_{1}, \mathbf{b}_{2}$ and the parameters are $R P_{\mathbf{b}_{1}}=R P_{\mathbf{b}_{2}}=1, \delta_{\mathbf{b}_{1}}=\delta_{\mathbf{b}_{2}}=0.95, T_{\mathbf{b}_{1}}=9, T_{\mathbf{b}_{2}}=12, R P_{\mathbf{s}}=0$, $\delta_{\mathbf{s}}=.95, T_{\mathbf{s}}=12$, and $d=1$. Agent $\mathbf{b}_{1}$ is indifferent at $t \geq 8$ between to be matched and not to be. Indeed, if she is matched at $t=8$, then, if she opens the negotiation at $t=9$, she makes exit and therefore she gains a utility of zero, if instead $\mathbf{s}$ opens the negotiation at $t=9$, s makes $\operatorname{match}\left(\mathbf{b}_{2}\right)$ and therefore $\mathbf{b}_{1}$ gains a utility of zero. On the other hand, if she is not matched at $t=8$, she gains a utility of zero. In the situation wherein $\mathbf{b}_{1}$ makes matchable(s) at $t=8$, $\mathbf{s}$ will make match $\left(\mathbf{b}_{1}\right)$ at such time point. Indeed, if $\mathbf{s}$ makes the offer $x_{\mathbf{s}}^{*}\left(8, \mathbf{b}_{2}\right) \simeq .1$ as prescribed by the strategies provided in Sections 2.2 and 5.1, then she gains $U_{\mathbf{s}}\left(x_{\mathbf{s}}^{*}\left(8, \mathbf{b}_{2}\right), 0\right) \simeq .1$, whereas, if $\mathbf{s}$ makes match $\left(\mathbf{b}_{1}\right)$, then she expects $\simeq$.44. Exactly, the computation of the expected utility of making $\operatorname{match}\left(\mathbf{b}_{1}\right)$ at $t=8$ is the following. If $\mathbf{s}$ acts at $t=9$ (with probability $.5)$, she will make match $\left(\mathbf{b}_{2}\right)$ and then with probability $.5 \mathbf{s}$ acts at $t=10$ offering $x_{\mathbf{s}}^{*}\left(10, \mathbf{b}_{2}\right)=.05$ and with probability $.5 \mathbf{b}_{2}$ acts at $t=10$ offering $x_{\mathbf{b}_{2}}^{*}(10)=.95$; since these agreements are reached at $t=11$, their utility must be discounted by $\left(\delta_{\mathbf{s}}\right)^{2}$. If $\mathbf{b}_{1}$ acts at $t=9$ (with probability .5), she will make exit and s will make match $\left(\mathbf{b}_{2}\right)$ at $t=10$; in this case with probability $.5 \mathbf{s}$ acts at $t=11$ offering $x_{\mathbf{s}}^{*}\left(11, \mathbf{b}_{2}\right)=1$ and with probability $.5 \mathbf{b}_{2}$ acts at $t=11$ offering $x_{\mathbf{b}_{2}}^{*}(10)=0$; since these agreements are reached at $t=12$, their utility must be discounted by $\left(\delta_{\mathbf{s}}\right)^{3}$.

The above equilibrium is non-reasonable. Notice that $\mathbf{s}$ takes benefit from the presence of $\mathbf{b}_{1}$ even if $\mathbf{b}_{1}$ is not in competition with $\mathbf{b}_{2}$. Indeed, $\mathbf{b}_{1}$ will never reach any agreement with $\mathbf{s}$, her deadline being expired. The presence of a buyer should affect the equilibrium strategies only if she is "really" in competition with 

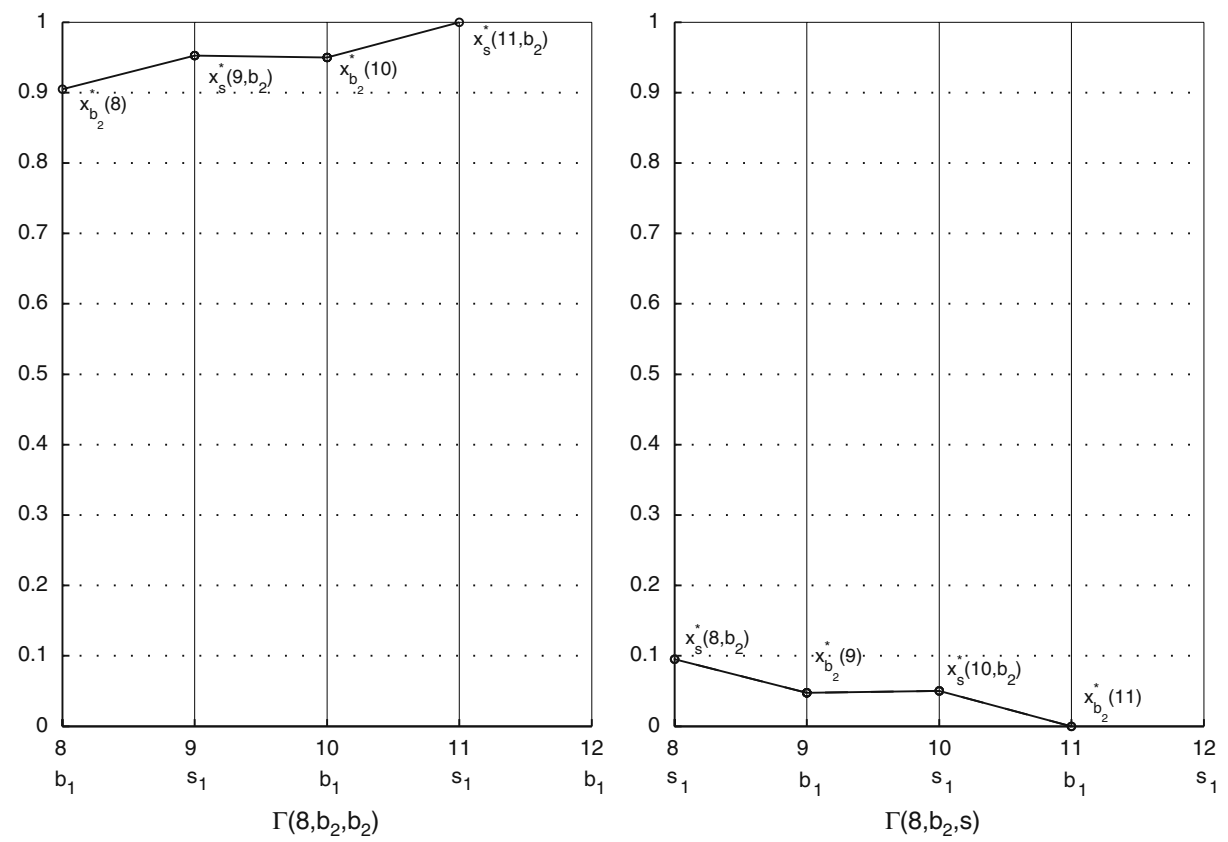

Fig. 5 Subgames $\Gamma\left(8, \mathbf{b}_{2}, \mathbf{b}_{2}\right)$ and $\Gamma\left(8, \mathbf{b}_{2}, \mathbf{s}\right)$ with $\mathbf{b}_{1}, \mathbf{b}_{2}$ where $R P_{\mathbf{b}_{1}}=R P_{\mathbf{b}_{2}}=1, \delta_{\mathbf{b}_{1}}=\delta_{\mathbf{b}_{2}}=.95$, $T_{\mathbf{b}_{1}}=9, T_{\mathbf{b}_{2}}=12, R P_{\mathbf{s}}=0, \delta_{\mathbf{s}}=.95, T_{\mathbf{s}}=12$, and $d=1$

the others. Technically speaking, a buyer should affect the equilibrium strategies only if she expects to gain a strictly positive utility. Furthermore, the equilibrium non-uniqueness is a crucial problem in an electronic market, where agents can be developed by different designers. Consider the previous example: the strategy of $\mathbf{b}_{1}$ after her deadline is fundamental for the determination of the sequence of the optimal offers. Since $\mathbf{b}_{1}$ has different optimal strategies after $T_{\mathbf{b}_{1}}$ and each of these strategies induce different optimal strategies for $\mathbf{b}_{2}$ and $\mathbf{s}$ at $t<T_{\mathbf{b}_{1}}$, agents could follow different equilibria. As a result, inefficiency raises.

Now we consider the problem to remove equilibria different from those prescribed by Theorem 5.1. In order for the protocol presented in Section 3.1 to admit a unique equilibrium, we need that any buyer strictly prefers not to start a negotiation rather than to start a negotiation from which she expects to reach the outcome No Agreement with probability equal to 1 . Technically speaking, we need to remove indifference from buyers' actions so that buyers will always employ a unique strategy. This condition can be easily achieved by introducing costs in negotiation: anytime a buyer $\mathbf{b}_{i}$ is matched by $\mathbf{s}, \mathbf{b}_{i}$ must pay a strictly positive fee $f$ to the negotiation platform on which the market is hosted. The value of $f$ will be negligible with respect to the utility expected by agents, otherwise agents have incentives to migrate on other negotiation platforms. For symmetry, we require that also s pays $f$; in this case we require that she pays $f$ only when she is not matched and makes match $(\cdot)$. Although our enrichment aims at assuring the existence of a unique equilibrium outcome, it 
"naturally" makes the proposed model closer to real-world applications. Indeed, it is reasonable that in real-world applications agents pay some fees to the negotiation platform for the transactions they carry on.

The fee $f$ is a little amount of money that reduces the utility of the agents involved in the negotiation (both of the two agents that have reached an agreement and of all the other buyers that have been matched during the negotiation). Formally, the utility of reaching an agreement $(x, t)$ for the agents is:

$$
\begin{aligned}
U_{\mathbf{b}_{i}}(x, t) & =\left\{\begin{array}{ll}
\left(R P_{\mathbf{b}}-x\right) \cdot \delta_{\mathbf{b}_{i}}^{t}-f \cdot k_{\mathbf{b}_{i}} & \text { if } A_{\mathbf{b}_{i}} \leq t \leq T_{\mathbf{b}_{i}} \\
-1-f \cdot k_{\mathbf{b}_{i}} & \text { otherwise }
\end{array},\right. \\
U_{\mathbf{s}}(x, t) & = \begin{cases}\left(x-R P_{\mathbf{s}}\right) \cdot \delta_{\mathbf{s}}^{t}-f \cdot k_{\mathbf{s}} & \text { if } A_{\mathbf{s}} \leq t \leq T_{\mathbf{s}} \\
-1-f \cdot k_{\mathbf{s}} & \text { otherwise }\end{cases}
\end{aligned}
$$

where $k_{\mathbf{b}_{i}}$ denotes the number of times that $\mathbf{b}_{i}$ has been matched by $\mathbf{s}$ and $k_{\mathbf{s}}$ is the number of times that $\mathbf{s}$ has made $\operatorname{match}(\cdot)$ at a time point where she was not matched; the utility of NoAgreement for an agent $i$ is $-f \cdot k_{i}$. Notice that if an agent $i$ has not been matched, i.e., $k_{i}=0$, her utility is 0 .

In the general case the introduction of $f>0$ alters agents' equilibrium strategies with respect to the ones prescribed in the previous section. However, in realworld applications $f \cdot k_{\mathbf{s}}$ will be negligible with respect to $\left(x_{\mathbf{s}}^{*}\left(t, \mathbf{b}_{i}\right)-R P_{\mathbf{s}}\right) \cdot \delta_{\mathbf{s}}^{t}$ at the equilibrium. Analogously, $f \cdot k_{\mathbf{b}_{i}}$ will be negligible with respect to $\left(R P_{\mathbf{b}_{i}}-x_{\mathbf{b}_{i}}^{*}(t)\right) \cdot \delta_{\mathbf{s}}^{t}$. It can be shown that there exists an upper bound $\bar{f}$ such that the strategies prescribed in the previous section hold to be of equilibrium when $0 \leq f<\bar{f}$. The computation of the exact upper bound $\bar{f}$ is involved. However, an easily computable upper bound $(<\bar{f})$ for $f$ is expressed in the following proposition.

Proposition 5.2 With utility functions (1) and (2), the strategies stated in Theorem 5.1 are of equilibrium when:

$$
0 \leq f<\frac{1}{2} \cdot\left(R P_{\mathbf{b}}-R P_{\mathbf{s}}\right) \cdot\left(1-\delta_{\mathbf{s}}\right) \cdot\left(\min \left\{\delta_{\mathbf{b}_{1}}, \delta_{\mathbf{b}_{2}}, \delta_{\mathbf{s}}\right\}\right)^{\bar{T}} .
$$

Proof (When $f=0$, the utility functions (1) and (2) are exactly the original ones, and therefore the result is obvious.) We initially notice that on the equilibrium path $k_{\mathbf{s}}=1$ (s makes only once match $(\cdot)$ at a time point where she is not matched), while the values of $k_{\mathbf{b}_{1}}$ and $k_{\mathbf{b}_{2}}$ depend on the parameters of the game. It can be observed that any buyer $\mathbf{b}_{i}$ expects at $t$ a utility larger than $\frac{1}{2} U_{\mathbf{b}_{i}}\left(\left(R P_{\mathbf{b}}\right)_{\leftarrow \mathbf{s}}, t+d\right)$ from starting a negotiation at $t+d$. This is because:

- with probability $.5 \mathbf{b}_{i}$ opens the negotiation at $t+d$,

$-x_{\mathbf{b}_{i}}^{*}(t+d) \leq\left(R P_{\mathbf{b}}\right)_{\leftarrow \mathbf{s}}$ for any time point $t+d \leq \bar{T}_{\mathbf{b}_{i}}-1$,

- $x_{\mathbf{b}_{i}}^{*}(t+d)$ will be accepted by $\mathbf{s}$ at $t+d+1$.

By trivial mathematics, it can be shown that $\frac{1}{2} \cdot\left(R P_{\mathbf{b}}-R P_{\mathbf{s}}\right) \cdot\left(1-\delta_{\mathbf{s}}\right) \cdot\left(\min \left\{\delta_{\mathbf{b}_{1}}, \delta_{\mathbf{b}_{2}}\right.\right.$, $\left.\left.\delta_{\mathbf{s}}\right\}\right)^{\bar{T}}<\frac{1}{2} U_{\mathbf{b}_{i}}\left(\left(R P_{\mathbf{b}}\right)_{\leftarrow \mathbf{s}}, t+d\right)$. Furthermore, it can be shown that the utility $\mathbf{s}$ expects to gain from starting a negotiation is strictly larger that $\frac{1}{2} U_{\mathbf{b}_{i}}\left(\left(R P_{\mathbf{b}}\right)_{\leftarrow \mathbf{s}}, \bar{T}\right)$. Indeed, $\mathbf{s}$ expects at least a utility equal to $\frac{1}{2} U_{\mathbf{s}}\left(R P_{\mathbf{b}}, \bar{T}\right)$ and, by trivial mathematics, it can be shown that $\frac{1}{2} \cdot\left(R P_{\mathbf{b}}-R P_{\mathbf{s}}\right) \cdot\left(1-\delta_{\mathbf{s}}\right) \cdot\left(\min \left\{\delta_{\mathbf{b}_{1}}, \delta_{\mathbf{b}_{2}}, \delta_{\mathbf{s}}\right\}\right)^{\bar{T}}<\frac{1}{2} U_{\mathbf{s}}\left(R P_{\mathbf{b}}, \bar{T}\right)$. 
Finally, the range stated in the proposition is always non-empty since for any value of the parameters the inequality $\frac{1}{2} \cdot\left(R P_{\mathbf{b}}-R P_{\mathbf{s}}\right) \cdot\left(1-\delta_{\mathbf{s}}\right) \cdot\left(\min \left\{\delta_{\mathbf{b}_{1}}, \delta_{\mathbf{b}_{2}}, \delta_{\mathbf{s}}\right\}\right)^{\bar{T}}>0$ holds.

Proposition 5.2 states that agents' equilibrium strategies when $f$ belongs to the given range are the same ones when $f=0$. This allows one no to take into account the exact value of $f$ in the computation the equilibrium. When $f$ is larger than the upper bound stated in Proposition 5.2 instead, agents' equilibrium strategies are generally different from those derived in the previous section. Nevertheless, the backward induction construction in these cases is an easy extension of the one discussed in the previous section. On the basis of Proposition 5.2 we state the following theorem.

Theorem 5.3 The outcome induced by the strategies prescribed in Table 3 is the unique subgame perfect equilibrium outcome of this game when $f$ belongs to the range stated in Proposition 5.2 but $f=0$ (except for a null measure subset of the space of the parameters).

Proof We divide the proof in two parts. In the first part we consider the situation wherein the non-matched buyers use at the first stage of every time point the strategy we have assigned them in the previous section, i.e., a buyer $\mathbf{b}_{i}$ makes matchable(s) if $t<\min \left\{T_{\mathbf{b}_{i}}, \bar{T}\right\}-d$ and nonmatchable otherwise, whereas in the second part we consider the situation where they use a different strategy.

Consider the first case. As we showed in Section 2.2, in order to analyze the multiplicity of equilibria, we can limit our analysis to the actions that are indifferent for the agents to their optimal actions on the equilibrium path. The actions indifferent for the buyers are exactly the same considered in Section 2.2: to accept $\left(x_{\mathbf{b}_{i}}^{*}(t+1)\right)_{\leftarrow \mathbf{b}_{i}}$ and to reject it to make her optimal action between offer $\left(x_{\mathbf{b}_{i}}^{*}(t+1)\right)$ and exit. As we showed in Section 2.2, no equilibrium can be if $\mathbf{b}_{i}$ rejects $\left(x_{\mathbf{b}_{i}}^{*}(t+1)\right)_{\leftarrow \mathbf{b}_{i}}$. The actions indifferent for the seller are similar to the ones considered in Section 2.2: to accept $\left(e_{\mathbf{s}}^{*}\left(t+1, \mathbf{b}_{i}\right)\right)_{\leftarrow \mathbf{s}}$ and to reject it to make the best among $\operatorname{offer}\left(x_{\mathbf{s}}^{*}\left(t+1, \mathbf{b}_{i}\right)\right), \operatorname{match}(\cdot)$, and exit. Similarly to what we showed in Section 2.2, it can be shown now that no equilibrium can be if $\mathbf{s}$ rejects $\left(e_{\mathbf{s}}^{*}\left(t+1, \mathbf{b}_{i}\right)\right)_{\leftarrow \mathbf{s}}$. Instead, in the situation wherein $\operatorname{offer}\left(x_{\mathbf{s}}^{*}\left(t+1, \mathbf{b}_{i}\right)\right)$ and match $(\cdot)$ are indifferent for $\mathbf{s}$, two equilibrium outcomes are possible: $\mathbf{s}$ can make indifferently $\operatorname{offer}\left(x_{\mathbf{s}}^{*}\left(t+1, \mathbf{b}_{i}\right)\right)$ and $\operatorname{match}(\cdot)$. However, this happens only for a null measure subset of the space of the parameters, and therefore it is negligible.

Consider the second case. We must focus on the situation wherein making matchable(s) or nonmatchable is indifferent for a buyer $\mathbf{b}_{i}$ before her deadline (after her deadline the buyer strictly prefers to be not matched due to $f$ ), but the use of these two actions induces different agents' strategies in the respective subgames (and therefore they could lead to different equilibrium outcomes). In this situation, it is possible to show that, if $\mathbf{b}_{i}$ makes nonmatchable (and therefore she uses a strategy different from the one prescribed in the previous section), no equilibrium there is. The proof uses trivial mathematics, but it is long. We show the principle on which the proof is based by illustrating an example. Consider the bargaining $\Gamma\left(0, \mathbf{b}_{1}, \mathbf{b}_{1}\right)$ depicted in Fig. 4 for $t \geq 8$. At $t=9$ the buyer $\mathbf{b}_{2}$ is indifferent between making matchable(s) and nonmatchable. Indeed, on the equilibrium path $\mathbf{b}_{1}$ offers $x_{\mathbf{b}_{1}}^{*}(8)$ at 
$t=8$ and $\mathbf{s}$ will accept such offer and therefore $\mathbf{b}_{2}$ gains a utility of zero independently of the action she actually makes. Be $\sigma^{*}$ an equilibrium strategy profile. Assume by contradiction that $\sigma^{*}$ prescribes that $\mathbf{b}_{2}$ makes nonmatchable at $t=9$. Therefore, by backward induction, $\mathbf{b}_{1}$ 's optimal offer at $t=8$ is $R P_{\mathbf{s}}$. However, if $\mathbf{b}_{1}$ offers $R P_{\mathbf{s}}$ at $t=8, \mathbf{b}_{2}$ 's optimal action at $t=9$ is matchable(s), since $\mathbf{s}$ 's optimal action at $t=9$ is to reject the offer $R P_{\mathbf{s}}$ of $\mathbf{b}_{1}$ and to make match $\left(\mathbf{b}_{2}\right)$. We reach thus a contradiction. As a result, the considered $\sigma^{*}$ cannot be an equilibrium strategy profile. This reasoning can be applied iteratively from $t=T_{\mathbf{b}_{1}}$ back to $t=0$, showing that no equilibrium profile $\sigma^{*}$ can prescribe that $\mathbf{b}_{i}$ makes nonmatchable at $t<\min \left\{\bar{T}, T_{\mathbf{b}_{i}}\right\}-d$.

The simple modification of agents' utility we have introduced is satisfactory since both it assures the equilibrium to be unique and provides a model that is more adherent to real-world applications.

\section{Extending to settings with uncertainty}

In presence of incomplete information, it is customary in game theory to introduce probability distributions over the parameters that are not known by the agents. This leads the game to be with uncertain information. Then, by Harsanyi's transformation, the game is cast to an imperfect information game, where players can be of different types and uncertainty is over the opponent's type. Classic game theory provides an appropriate solution concept for extensive-form games with imperfect information, i.e., the sequential equilibrium [30], but no solving technique to find it. We recall that the backward induction method can be employed with success exclusively in the presence of complete information [14]. From [27] there is a long standing literature in computer science, e.g., [22, 28, 31], that studies algorithms to find Nash equilibria and refinements, e.g., sequential equilibria [33], searching in the space of the strategies. These algorithms have two main drawbacks that make them inapplicable in solving bargaining situations: they work only with games with finite strategies and they produce equilibrium strategies but not systems of beliefs.

The lack of a solving algorithm for games as bargaining has pushed researchers to game theoretically study each possible specific setting and develop relative algorithms. Nowadays, the analysis of bargaining with uncertain information is currently more a series of examples than a coherent set of results. Well known examples of alternating-offers settings studied in literature are: in [39] Rubinstein analyzes onesided uncertainty over the discount factors with only two types, in [6] Di Giunta and Gatti analyze in pure strategies one-sided uncertainty over deadlines, in [3] Chatterjee and Samuelson analyze uncertainty over reservation prices where the agents can be of two possible types, and in [11] Fatima et al. consider a slight variation of the alternating-offers protocol where there are multiple issues to negotiate and analyze uncertainty over the weights of the issues. An interested reader can find an exhaustive survey on bargaining with uncertain information in [4].

As a consequence, the extension of the results discussed in this paper to settings with uncertainty is a hard task to be tackled. Anyway, the results we presented here can significantly aid the study of those settings. Indeed, as is showed in [20], the study 
of uncertainty settings is strongly based on the complete-information solution. In particular, in [20] the authors show that when the deadlines are uncertain the agents' equilibrium strategies prescribe that rational agents should randomize over the offers that are of equilibrium with complete information. The same result can be easily extended to general settings [2]. This requires the computation of the equilibrium offers with complete information of all the possible games for each possible agents' type profile. This task can be accomplished with the results presented in this paper.

\section{Conclusions and future works}

Automated agents were suggested as a way to facilitate increasingly efficient negotiations. Their automation is commonly carried out by employing game theoretical tools and is currently a challenging issue for artificial intelligence research. In this paper we have taken into account the best-known negotiation protocol for the most common economic transaction, i.e., alternating-offers bargaining. A vast literature analyzes this protocol and its refinements. In particular, several works study bargaining settings where agents negotiate over multiple issues and/or where the value of one or more parameters related to agents' utility functions is uncertain. A crucial setting that currently does not find a satisfactory analysis in the literature is the setting where the two bargainers act in a market of bargaining agents. This setting is of paramount importance, since the presence of multiple bargaining agents is common in real-world electronic markets and the lack of a satisfactory model for this setting prevents an effective employment of autonomous agents. In order to cope with this problem, we resort to classical results from microeconomic/game theory literature. Specifically, we model the matching problem and we introduce the outside option in the alternatingoffers protocol, i.e., the option available to an agent of leaving the negotiation she is carrying out to start a new negotiation.

There are essentially two original contributions in this paper. First, we provided a satisfactory bargaining protocol to capture the situation in which there are multiple seller and buyers: agents can have different parameters, have deadlines, and the seller agents have the option of leaving the negotiation they are carrying out to choose a specific buyer with whom they will start a new negotiation. Second, we analyzed the protocol, deriving equilibrium strategies. We showed that when the outside option is inhibited, the problem can be reduced to one of matching and can be solved by applying Gale-Shapley's stable marriage algorithm. We derived also computational complexity results. When there is only one seller and the outside option is not inhibited, we showed that the protocol can admit multiple equilibria, among which only one is reasonable. We provided an algorithm to compute this equilibrium and showed that the introduction of fees for the players during the negotiation can lead to remove non-reasonable equilibria.

This paper will breed a large amount of future works. At first, our interest is in analyzing two-sided competition, where multiple buyers and multiple sellers can be concurrently involved in bargaining. Then, our intention is to exploit results known in literature on the presence of uncertainty in bargaining to study the effects of uncertainty in markets of bargaining agents. A further work we are carrying 
out concerns the development of electronic platforms to support game theoretical bargaining agents.

Acknowledgements I am grateful to Nicola Basilico, Alex Davidson, Francesco Di Giunta, Roberto Lucchetti, and Fabio Pellicioli for the fruitful discussions.

\section{Appendix}

\section{A Equilibrium strategies in the general case with one-side competition}

In this section we consider the situation wherein a number $n$ of buyers populates the market, buyers can have different reservation prices, and they can enter the market at different time points. We discuss these extensions by degrees in Appendices A.1, A.2, and A.3, and we report the equilibrium strategies and the solving algorithm in Appendix A.4.

\section{A.1 More than two buyers}

We extend the setting considered in Section 5 by introducing multiple buyers. The presence of a number $m$ of buyers makes the equilibrium strategies more intricate, but the solution can be provided similarly to the case with two buyers. The time point from which the backward induction method must be applied is $\bar{T}=\min \left\{\max _{i \in\{1, \ldots, m\}}\left\{T_{\mathbf{b}_{i}}\right\}, T_{\mathbf{s}}\right\}$. In the first step of each time point $t$, each non-matched buyer $\mathbf{b}_{i}$ will make matchable(s) if $t<\min \left\{T_{\mathbf{b}_{i}}, \bar{T}\right\}-d$ and nonmatchable otherwise. Analogously, in the second step of each time point $t$ each non-matched buyer $\mathbf{b}_{i}$ will make wait if $t<\min \left\{T_{\mathbf{b}_{i}}, \bar{T}\right\}-d$ and exit otherwise. We consider now agents' strategies during the negotiation. As in the case with two buyers, the equilibrium strategies of $\mathbf{b}_{i}$ prescribe that after $t=\min \left\{T_{\mathbf{b}_{i}}, \bar{T}\right\}$ she makes exit and from $t=$ $\min \left\{T_{\mathbf{b}_{i}}, \bar{T}\right\}-1$ to $t=0$ she makes the lowest offer that $\mathbf{s}$ would accept at the next time point (otherwise she accepts an offer not worse than making her optimal action). The equilibrium strategies of $\mathbf{s}$ can be produced at each time point searching for the action, among all the available ones, that maximizes her equivalent value. Precisely, the actions to be considered here are: to make the highest offer that her current opponent would accept at the next time point and to match any other possible opponent $\mathbf{b}_{i}$ such that $\mathbf{b}_{i}$ has made matchable(s), namely, all those $\mathbf{b}_{i}$ s such that $t<\min \left\{T_{\mathbf{b}_{i}}, \bar{T}\right\}-d$. For the sake of presentation, we introduce the following notation. We denote by $E_{m}(t)$ the set composed of the equivalent values for $\mathbf{s}$ of making match $\left(\mathbf{b}_{j}\right)$ at $t$ when $\mathbf{s}$ is not matched and $t<\min \left\{T_{\mathbf{b}_{j}}, \bar{T}\right\}-d$; by $e_{m}(t)$ the maximum element of $E_{m}(t)$; and by $\mathbf{b}_{m}(t)$ the buyer $\mathbf{b}_{j}$ to match such that the equivalent value is maximum. Formally, they are:

$$
\begin{aligned}
& -\quad E_{m}(t)=\left\{\left(\frac{1}{2} x_{\mathbf{b}_{j}}^{*}(t+d)+\frac{1}{2} e_{\mathbf{s}}^{*}\left(t+d, \mathbf{b}_{j}\right)\right)_{\leftarrow d[\mathbf{s}]}: \forall j \operatorname{such} \text { that } t<\min \left\{T_{\mathbf{b}_{j}}, \bar{T}\right\}-d\right\}, \\
& -\quad e_{m}(t)=\max _{j}\left\{E_{m}(t)\right\}, \\
& -\quad \mathbf{b}_{m}(t)=\arg \max _{j}\left\{E_{m}(t)\right\} .
\end{aligned}
$$


We denote by $E_{m}\left(t, \mathbf{b}_{i}\right)$ the set defined as $E_{m}(t)$ but subject to $j \neq i: E_{m}\left(t, \mathbf{b}_{i}\right)$ is composed of the equivalent values for $\mathbf{s}$ of making $\operatorname{match}\left(\mathbf{b}_{j}\right)$ at $t$ when she is negotiating with $\mathbf{b}_{i}$ and $t<\min \left\{T_{\mathbf{b}_{j}}, \bar{T}\right\}-d$. In an analogous way, we define $e_{m}\left(t, \mathbf{b}_{i}\right)$ and $\mathbf{b}_{m}\left(t, \mathbf{b}_{i}\right)$. Formally, they are:

$$
\begin{array}{ll}
- & E_{m}\left(t, \mathbf{b}_{i}\right)=\left\{\left(\frac{1}{2} x_{\mathbf{b}_{j}}^{*}(t+d)+\frac{1}{2} e_{\mathbf{s}}^{*}\left(t+d, \mathbf{b}_{j}\right)\right)_{\leftarrow d[\mathbf{s}]}: \forall j \text { such that } j \neq i\right. \text { and } \\
& \left.t<\min \left\{T_{\mathbf{b}_{j}}, \bar{T}\right\}-d\right\}, \\
-\quad & e_{m}\left(t, \mathbf{b}_{i}\right)=\max _{j}\left\{E_{m}\left(t, \mathbf{b}_{i}\right)\right\}, \\
-\quad \mathbf{b}_{m}\left(t, \mathbf{b}_{i}\right)=\arg \max _{j}\left\{E_{m}\left(t, \mathbf{b}_{i}\right)\right\} .
\end{array}
$$

In the worst case, the optimal action of $\mathbf{s}$ at $t$ in $\Gamma\left(t, \mathbf{b}_{i}, \mathbf{s}\right)$ can be computed through a linear search among $n$ non-ordered elements: $n-1$ elements composing the set $E_{m}\left(t, \mathbf{b}_{i}\right)$ and the offer $\left(x_{\mathbf{b}_{i}}^{*}(t+1)\right)_{\leftarrow \mathbf{b}_{i}}$. Since we need to compute the optimal action of $\mathbf{s}$ at $t$ in each possible $\Gamma\left(t, \mathbf{b}_{i}, \mathbf{s}\right)$ with $i \in\{1, \ldots, n\}$, the computation of the solution would require $n$ maximizations among $n$ non-ordered elements at each time point $t$, formally, $O\left(n^{2} \cdot \bar{T}\right)$. However, such a computation can be achieved in time linear in $n$. Indeed, considering $e_{m}\left(t, \mathbf{b}_{i}\right) \mathrm{s}$ for all the possible values of $i$, we have that at $t$ they can assume at most two different values. Exactly, their values can be either the first or the second element of $E_{m}(t)$ once this set was ordered from the maximum value to the minimum one. Among all the possible buyers to match, $\mathbf{s}$ will always prefer to match the buyer corresponding to the maximum of $E_{m}(t)$, but when such buyer is the buyer with whom she is negotiating (in this situation $\mathbf{s}$ will prefer to match the buyer corresponding to the greatest equivalent value of $E_{m}(t)$ once the maximum was removed). The first two greatest elements in $E_{m}(t)$ can be found in time asymptotically linear in $n$ (their computation requires two linear searches in a non-ordered set of $n$ elements). Once these values are found, we can compute the optimal actions of s searching among two elements: the best buyer to match, i.e., $\mathbf{b}_{m}\left(t, \mathbf{b}_{i}\right)$, and greatest offer that her opponent would accept at the next time point, i.e., $\left(x_{\mathbf{b}_{i}}^{*}(t+1)\right)_{\leftarrow \mathbf{b}_{i}}$. Since this search must be repeated at every time point of the negotiation, it trivially follows that the computational complexity required to produce agents' equilibrium strategies is $O(n \cdot \bar{T})$.

\section{A.2 Different reservation prices}

We extend the setting considered in the previous section by allowing buyers to have different reservation prices. In this setting the solution presents some complications. Trivially, in this setting we must consider only the buyers whose reservation price is larger than the reservation price of the seller, namely, only the buyers $\mathbf{b}_{i}$ s such that $R P_{\mathbf{b}_{i}}>R P_{\mathbf{s}}$. Indeed, all the $\mathbf{b}_{i}$ s with $R P_{\mathbf{b}_{i}} \leq R P_{\mathbf{s}}$ will make nonmatchable. Less trivially, in this setting two anomalies can be:

1. the optimal action of $\mathbf{b}_{i}$ at $t$ can be to make an offer that is unacceptable for $\mathbf{s}$ at $t+1$ (and then the agreement will be delayed), 
2. at $t$ a buyer $\mathbf{b}_{i}$ can expect negative utility from starting a negotiation at $t+d$ (and then she will make nonmatchable before $\left.t=\min \left\{T_{\mathbf{b}_{i}}, \bar{T}\right\}-d\right)$.

We report an example wherein both these situations coexist. Consider a setting with two buyers, $\mathbf{b}_{1}$ and $\mathbf{b}_{2}$, where $R P_{\mathbf{b}_{1}}>R P_{\mathbf{b}_{2}}$. Consider the game $\Gamma\left(t+d, \mathbf{b}_{2}, \mathbf{b}_{2}\right)$. It can be possible that the optimal action of $\mathbf{s}$ at $t+d+1$ is $\operatorname{match}\left(\mathbf{b}_{1}\right)$ with an equivalent value $e_{\mathbf{s}}^{*}\left(t+d+1, \mathbf{b}_{2}\right)$ such that $\left(e_{\mathbf{s}}^{*}\left(t+d+1, \mathbf{b}_{2}\right)\right)_{\leftarrow \mathbf{s}}>R P_{\mathbf{b}_{2}}$. Then $\mathbf{b}_{2}$ does not offer $\left(e_{\mathbf{s}}^{*}\left(t+d+1, \mathbf{b}_{2}\right)\right)_{\leftarrow \mathbf{s}}$ at $t+d$ (as instead prescribed by the strategies provided in Section 5), but she makes an offer unacceptable for $\mathbf{s}$. If in this situation the optimal action of $\mathbf{s}$ at $t+d$ in $\Gamma\left(t+d, \mathbf{b}_{2}, \mathbf{s}\right)$ is match $\left(\mathbf{b}_{1}\right)$ or to offer something unacceptable for $\mathbf{b}_{2}$, at $t \mathbf{b}_{2}$ expects a utility equal to $-f$ from starting to negotiate with $\mathbf{s}$ at $t+d$. Notice that expecting a utility of zero from starting a negotiation at $t+d$ does not entail expecting a utility of zero from starting a negotiation at any other time point. For instance, in the previous setting $\mathbf{b}_{2}$ could expect positive utility from starting to negotiate at $t=0$.

We find now the optimal actions of the agents when $\mathbf{b}_{i}$ 's optimal offers are unacceptable for $\mathbf{s}$. A buyer $\mathbf{b}_{i}$ makes an unacceptable offer at $t$ when $\left(e_{\mathbf{s}}^{*}\left(t+1, \mathbf{b}_{i}\right)\right)_{\leftarrow \mathbf{b}_{i}}>$ $R P_{\mathbf{b}_{i}}$, namely, when $\mathbf{b}_{i}$ gains negative utility from making the lowest offer that $\mathbf{s}$ will be accept. In this case, we set, for the sake of simplicity, the optimal offer of $\mathbf{b}_{i}$ equal to $R P_{\mathbf{b}_{i}}$. Formally, we write $x_{\mathbf{b}_{i}}^{*}(t)=\min \left\{\left(e_{\mathbf{s}}^{*}\left(t+1, \mathbf{b}_{i}\right)\right)_{\leftarrow} \mathbf{b}_{i}, R P_{\mathbf{b}_{i}}\right\}$. Notice that, when the arrival times of the buyers are equal, $\mathbf{s}$ will not make an offer unacceptable for $\mathbf{b}_{i}$. This is because s will always prefer to make match $(\cdot)$ rather than to make an offer unacceptable for $\mathbf{b}_{i}$.

A buyer $\mathbf{b}_{i}$ expects at $t$ negative utility from starting a negotiation at $t+d$ if $x_{\mathbf{b}_{i}}^{*}(t)=R P_{\mathbf{b}_{i}}$ (we discuss the proof in Appendix B.2). Therefore, $\mathbf{b}_{i}$ makes matchable at $t<\min \left\{T_{\mathbf{b}_{i}}, \bar{T}\right\}-d$ if $x_{\mathbf{b}_{i}}^{*}(t+d)<R P_{\mathbf{b}_{i}}$ and makes nonmatchable otherwise. The equilibrium strategies can be found constraining the equivalent values that compose the set $E_{m}\left(t, \mathbf{b}_{i}\right)$ : we require that such set is composed of the equivalent values of matching only the buyers $\mathbf{b}_{i}$ s such that $x_{\mathbf{b}_{i}}^{*}(t+d)<R P_{\mathbf{b}_{i}}$. Notice that the situation wherein the buyers can have different reservation prices does not raise the computational complexity of finding the solution, i.e., it is $O(n \cdot \bar{T})$.

\section{A.3 Different arrival times}

We extend the setting considered in the previous section by allowing buyers to enter the market at different time points. (Without loss of generality, we consider exclusively time points $t$ s such that $t \geq A_{\mathbf{s}}$.) Trivially, the equilibrium strategies of $\mathbf{b}_{i}$ are defined only at $A_{\mathbf{b}_{i}} \leq t$ and the set $E_{m}\left(t, \mathbf{b}_{i}\right)$ is composed only of the equivalent values for $\mathbf{s}$ of matching $\mathbf{b}_{j}$ with $A_{\mathbf{b}_{j}} \leq t$. Therefore, the formula of $E_{m}\left(t, \mathbf{b}_{i}\right)$ is:

$$
\begin{gathered}
E_{m}\left(t, \mathbf{b}_{i}\right)=\left\{\left(\frac{1}{2} x_{\mathbf{b}_{j}}^{*}(t+d)+\frac{1}{2} e_{\mathbf{s}}^{*}\left(t+d, \mathbf{b}_{j}\right)\right)_{\leftarrow d[\mathbf{s}]}: \text { such that } j \neq i,\right. \\
\left.A_{\mathbf{b}_{j}} \leq t<T_{\mathbf{b}_{j}}-d, \text { and } x_{\mathbf{b}_{j}}^{*}(t+d)<R P_{\mathbf{b}_{j}}\right\} .
\end{gathered}
$$

This is to say that $\mathbf{s}$ can match a buyer only if this has already entered the market. Less trivially, in this setting two anomalies can be: 
- if $\mathbf{s}$ is matched with $\mathbf{b}_{i}$, it is possible that the optimal action of $\mathbf{s}$ is to make an offer that is unacceptable for $\mathbf{b}_{i}$,

- $\quad$ if $\mathbf{s}$ is not matched, it is possible that her optimal action is to wait rather than to make $\operatorname{match}(\cdot)$.

We report two examples. Consider a setting with two buyers, $\mathbf{b}_{1}$ and $\mathbf{b}_{2}$, where $R P_{\mathbf{b}_{2}}>R P_{\mathbf{b}_{1}}$ and $A_{\mathbf{b}_{2}}>A_{\mathbf{b}_{1}}>T_{\mathbf{b}_{2}}>T_{\mathbf{b}_{1}}$. Consider the game $\Gamma\left(A_{\mathbf{b}_{2}}, \mathbf{b}_{1}, \mathbf{s}\right)$. It can be possible that the optimal action of $\mathbf{s}$ at $t=A_{\mathbf{b}_{2}}$ is $\operatorname{match}\left(\mathbf{b}_{2}\right)$ with an equivalent value $e_{\mathbf{s}}^{*}\left(A_{\mathbf{b}_{2}}, \mathbf{b}_{1}\right)$ such that $\left(e_{\mathbf{s}}^{*}\left(A_{\mathbf{b}_{2}}, \mathbf{b}_{1}\right)\right)_{\leftarrow 2[\mathbf{s}]}>R P_{\mathbf{b}_{1}}$. At $t=A_{\mathbf{b}_{2}}-2$ the optimal action of $\mathbf{s}$ is to make an offer unacceptable for $\mathbf{b}_{1}$. Indeed, since $t<A_{\mathbf{b}_{2}}$, $\mathbf{s}$ cannot match $\mathbf{b}_{2}$ and, since $\left(e_{\mathbf{s}}^{*}\left(A_{\mathbf{b}_{2}}, \mathbf{b}_{1}\right)\right)_{\leftarrow 2[\mathbf{s}]}>R P_{\mathbf{b}_{1}}$, s prefers to wait for $t=A_{\mathbf{b}_{2}}$ and then to make $\operatorname{match}\left(\mathbf{b}_{2}\right)$ rather than to make any offer that $\mathbf{b}_{1}$ would accept at $t=A_{\mathbf{b}_{2}}-1$ (or to make exit). Consider now a setting with two buyers, $\mathbf{b}_{1}$ and $\mathbf{b}_{2}$, where $R P_{\mathbf{b}_{2}}>R P_{\mathbf{b}_{1}}$ and $A_{\mathbf{b}_{2}}>T_{\mathbf{b}_{2}}>A_{\mathbf{b}_{1}}>T_{\mathbf{b}_{1}}$. Consider the situation at $t<T_{\mathbf{b}_{1}}-d$ where $\mathbf{s}$ is not matched. It can be possible that the utility $\mathbf{s}$ gains from waiting for $A_{\mathbf{b}_{2}}$ and then making match $\left(\mathbf{b}_{2}\right)$ is larger than the utility $\mathbf{s}$ gains from negotiating with $\mathbf{b}_{i}$. Therefore s prefers to make wait rather than to make match $(\cdot)$.

We find now the optimal actions of the agents when s's optimal offers are unacceptable the matched buyer. $\mathbf{s}$ makes an unacceptable offer at $t$ when $\left(e_{\mathbf{s}}^{*}(t+\right.$ $\left.\left.2, \mathbf{b}_{i}\right)\right)_{\leftarrow 2[s]}>\max \left\{R P_{\mathbf{b}_{i}}, e_{m}\left(t, \mathbf{b}_{i}\right)\right\}$, namely, when $\mathbf{s}$ gains more from waiting for two time points rather than to make an acceptable offer or make match $\left(\mathbf{b}_{m}\left(t, \mathbf{b}_{i}\right)\right)$. In this case, we set, for the sake of simplicity, the optimal offer of $\mathbf{s}$ equal to $\left(e_{\mathbf{s}}^{*}\left(t+2, \mathbf{b}_{i}\right)\right)_{\leftarrow 2[s]}$. Formally, we write $e_{\mathbf{s}}^{*}\left(t, \mathbf{b}_{i}\right)=\max \left\{\left(x_{\mathbf{b}_{i}}^{*}(t)\right)_{\leftarrow \mathbf{b}_{i}}, e_{m}\left(t, \mathbf{b}_{i}\right),\left(e_{\mathbf{s}}^{*}(t+\right.\right.$ $\left.\left.\left.2, \mathbf{b}_{i}\right)\right)_{\leftarrow 2[\mathbf{s}]}\right\}$ and $x_{\mathbf{s}}^{*}\left(t, \mathbf{b}_{i}\right)=e_{\mathbf{s}}^{*}\left(t, \mathbf{b}_{i}\right)$ if $e_{\mathbf{s}}^{*}\left(t, \mathbf{b}_{i}\right) \neq e_{m}\left(t, \mathbf{b}_{i}\right)$ and $x_{\mathbf{s}}^{*}\left(t, \mathbf{b}_{i}\right)$ is not defined otherwise.

In order to capture the situation where a non-matched s prefers to wait for some time points rather than to match a buyer, we introduce the following two notations:

$$
\begin{aligned}
&-\quad E_{w}(t)=\{\left(\frac{1}{2} x_{\mathbf{b}_{j}}^{*}\left(A_{\mathbf{b}_{j}}+d\right)+\frac{1}{2} e_{\mathbf{s}}^{*}\left(A_{\mathbf{b}_{j}}+d, \mathbf{b}_{j}\right)\right)_{\leftarrow\left(A_{\mathbf{b}_{j}}-t+d\right)[\mathbf{s}]}: \\
&\left.\forall j \text { such that } t<A_{\mathbf{b}_{j}}\right\}, \\
&-\quad e_{w}(t)=\max _{j}\left\{E_{w}(t)\right\} .
\end{aligned}
$$

The set $E_{w}(t)$ is composed of the equivalent value for $\mathbf{s}$ of matching buyers that they are not present in the market at $t$. The value $e_{w}(t)$ is the maximum element of $E_{w}(t)$. Agent $\mathbf{s}$ will prefer to wait rather than to match a buyer when $e_{w}(t)>e_{m}(t)$. Notice that the situation wherein the buyers can enter the market at different time points does not raise the computational complexity of finding the solution, i.e., it is $O(n \cdot \bar{T})$.

\section{A.4 Equilibrium strategies and solving algorithm}

We summarize in Table 4 the optimal actions of the agents in any possible situation wherein the market can be. In what follows, we provide the formulas to compute the sequences $x_{\mathbf{b}_{i}}^{*}(t), x_{\mathbf{s}}^{*}\left(t, \mathbf{b}_{i}\right)$, and $e_{\mathbf{s}}^{*}\left(t, \mathbf{b}_{i}\right)$ for all $i \in\{1, \ldots, n\}$. 
Table 4 Agents' optimal actions in the general case for $t \geq A_{\mathbf{s}}$

\begin{tabular}{|c|c|c|c|c|}
\hline Situation & Stage & Agents & Time points & Optimal actions \\
\hline \multirow[t]{3}{*}{$\begin{array}{c}\mathbf{b}_{i}(\mathbf{s}) \text { is not } \\
\text { matched }\end{array}$} & 1 & $\mathbf{b}_{i}$ & Any & $\begin{array}{l}\text { matchable }(\mathbf{s}) \text { if } A_{\mathbf{b}_{i}} \leq t<\min \left\{T_{\mathbf{b}_{i}}, \bar{T}\right\} \\
\quad-d \text { and } x_{\mathbf{b}_{i}}^{*}(t+d)<R P_{\mathbf{b}_{i}}, \\
\text { nonmatchable otherwise }\end{array}$ \\
\hline & 2 & $\mathbf{b}_{i}$ & Any & $\begin{array}{l}\text { wait if } t<\min \left\{T_{\mathbf{b}_{i}}, \bar{T}\right\}-d \text {, } \\
\text { exit otherwise }\end{array}$ \\
\hline & & $\mathbf{s}$ & Any & $\begin{array}{l}\operatorname{match}\left(\mathbf{b}_{m}(t)\right) \text { if } e_{m}(t) \geq e_{w}(t) \\
\quad \text { else wait if } t<\bar{T}-d, \text { and } \\
\quad \text { exit otherwise }\end{array}$ \\
\hline $\mathbf{b}_{i}(\mathbf{s})$ is & 2 & $\mathbf{b}_{i}$ & Alternately & $\sigma_{\mathbf{b}_{i}}^{*}(t)$ defined in Appendix A.4 \\
\hline negotiating & 2 & $\mathbf{s}$ & Alternately & $\sigma_{\mathbf{s}}^{*}\left(t, \mathbf{b}_{i}\right)$ defined in Appendix A.4 \\
\hline
\end{tabular}

For all $i$ such that $\bar{T} \leq T_{\mathbf{b}_{i}}$ and $R P_{\mathbf{b}_{i}}>R P_{\mathbf{s}}$, and for all time points $t \geq$ $\max \left\{A_{\mathbf{b}_{i}}, A_{\mathbf{s}}\right\}$ :

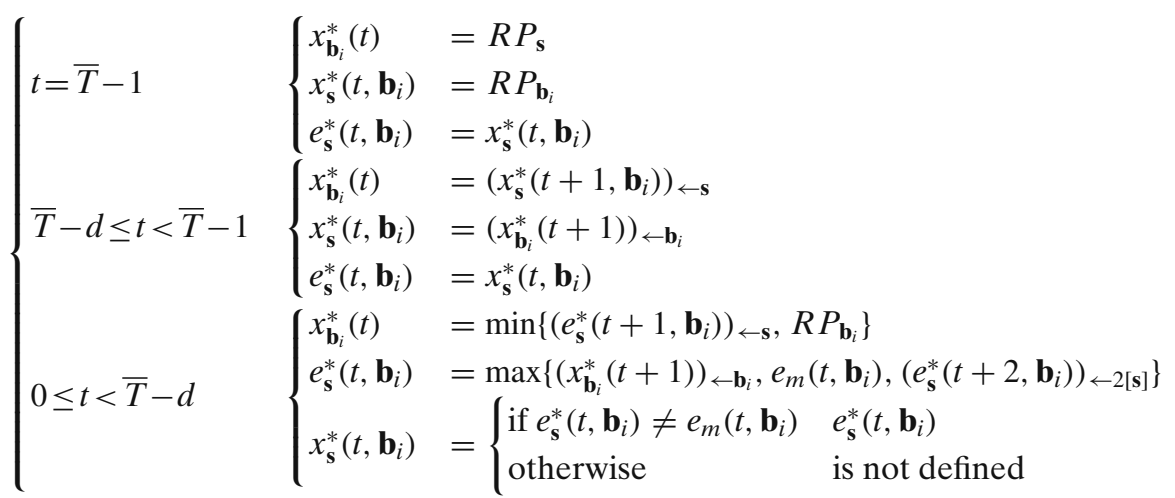

for all $i$ such that $\bar{T}-d \leq T_{\mathbf{b}_{i}}<\bar{T}$ and $R P_{\mathbf{b}_{i}}>R P_{\mathbf{s}}$, and for all time points $t \geq$ $\max \left\{A_{\mathbf{b}_{i}}, A_{\mathbf{s}}\right\}$ :

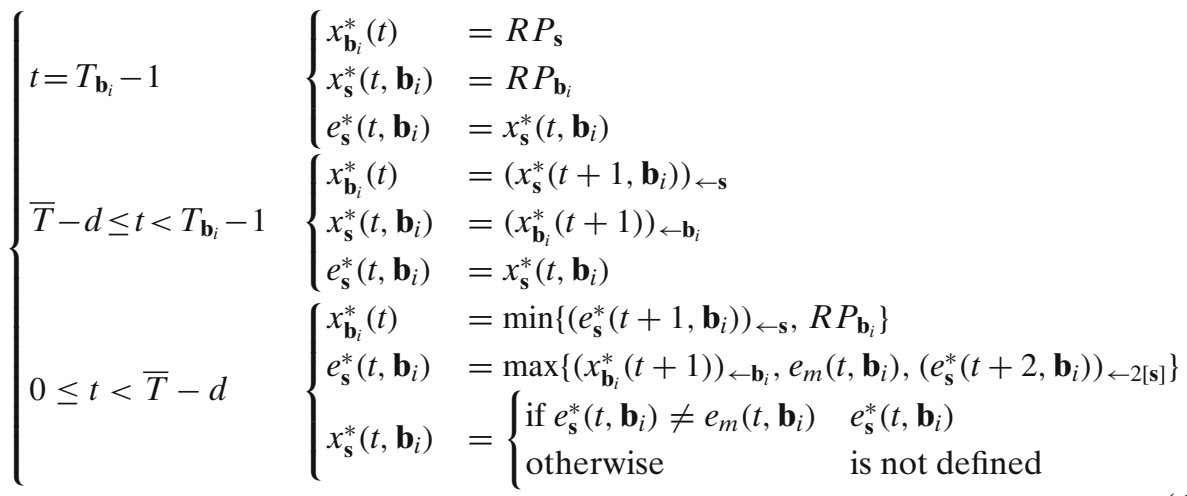


for all $i$ such that $T_{\mathbf{b}_{i}}<\bar{T}-d$ and $R P_{\mathbf{b}_{i}}>R P_{\mathbf{s}}$, and for all time points $t \geq$ $\max \left\{A_{\mathbf{b}_{i}}, A_{\mathbf{s}}\right\}$ :

$$
\begin{cases}T_{\mathbf{b}_{i}} \leq t<\bar{T}-d & \begin{cases}x_{\mathbf{b}_{i}}^{*}(t) & =\text { is not defined } \\ x_{\mathbf{s}}^{*}\left(t, \mathbf{b}_{i}\right) & =\text { is not defined } \\ e_{\mathbf{s}}^{*}\left(t, \mathbf{b}_{i}\right) & =\max \left\{e_{m}\left(t, \mathbf{b}_{i}\right), e_{w}(t)\right\}\end{cases} \\ 0 \leq t \leq T_{\mathbf{b}_{i}}-1 & \begin{cases}x_{\mathbf{b}_{i}}^{*}(t) & \min \left\{\left(e_{\mathbf{s}}^{*}\left(t+1, \mathbf{b}_{i}\right)\right)_{\leftarrow \mathbf{s}}, R P_{\mathbf{b}_{i}}\right\} \\ e_{\mathbf{s}}^{*}\left(t, \mathbf{b}_{i}\right) & =\max \left\{\left(x_{\mathbf{b}_{i}}^{*}(t+1)\right)_{\leftarrow \mathbf{b}_{i}}, e_{m}\left(t, \mathbf{b}_{i}\right),\left(e_{\mathbf{s}}^{*}\left(t+2, \mathbf{b}_{i}\right)\right)_{\leftarrow 2[\mathbf{s}]}\right\} \\ x_{\mathbf{s}}^{*}\left(t, \mathbf{b}_{i}\right) & = \begin{cases}\text { if } e_{\mathbf{s}}^{*}\left(t, \mathbf{b}_{i}\right) \neq e_{m}\left(t, \mathbf{b}_{i}\right) & e_{\mathbf{s}}^{*}\left(t, \mathbf{b}_{i}\right) \\ \text { otherwise } & \text { is not defined }\end{cases} \end{cases} \end{cases}
$$

The equilibrium strategies during the negotiations can be defined on the basis of the above sequences as follows.

For all $i$ such that $\bar{T} \leq T_{\mathbf{b}_{i}}$ and $R P_{\mathbf{b}_{i}}>R P_{\mathbf{s}}$, and for all time points $t \geq$ $\max \left\{A_{\mathbf{b}_{i}}, A_{\mathbf{s}}\right\}$ :

$$
\sigma_{\mathbf{b}_{i}}^{*}(t)= \begin{cases}t=0 & \begin{array}{l}
\text { offer }\left(\min \left\{R P_{\mathbf{b}_{i}}, x_{\mathbf{b}_{i}}^{*}(t)\right\}\right) \\
0<t<\bar{T}
\end{array} \\
\bar{T} \leq t \leq T_{\mathbf{b}_{i}} & \begin{cases}\text { if } \sigma_{\mathbf{s}}(t-1)=\text { offer }(x) \text { with } x \leq\left(x_{\mathbf{b}_{i}}^{*}(t)\right)_{\leftarrow \mathbf{b}_{i}} \quad \begin{array}{l}
\text { accept } \\
\text { otherwise }
\end{array} & \text { offer }\left(x_{\mathbf{b}_{i}}^{*}(t)\right) \\
\text { if } \sigma_{\mathbf{s}}(t-1)=\text { offer }(x) \text { with } x \leq R P_{\mathbf{b}_{i}} & \text { accept } \\
\text { otherwise } & \text { exit }\end{cases} \\
T_{\mathbf{b}_{i}<t} & \text { exit }\end{cases}
$$

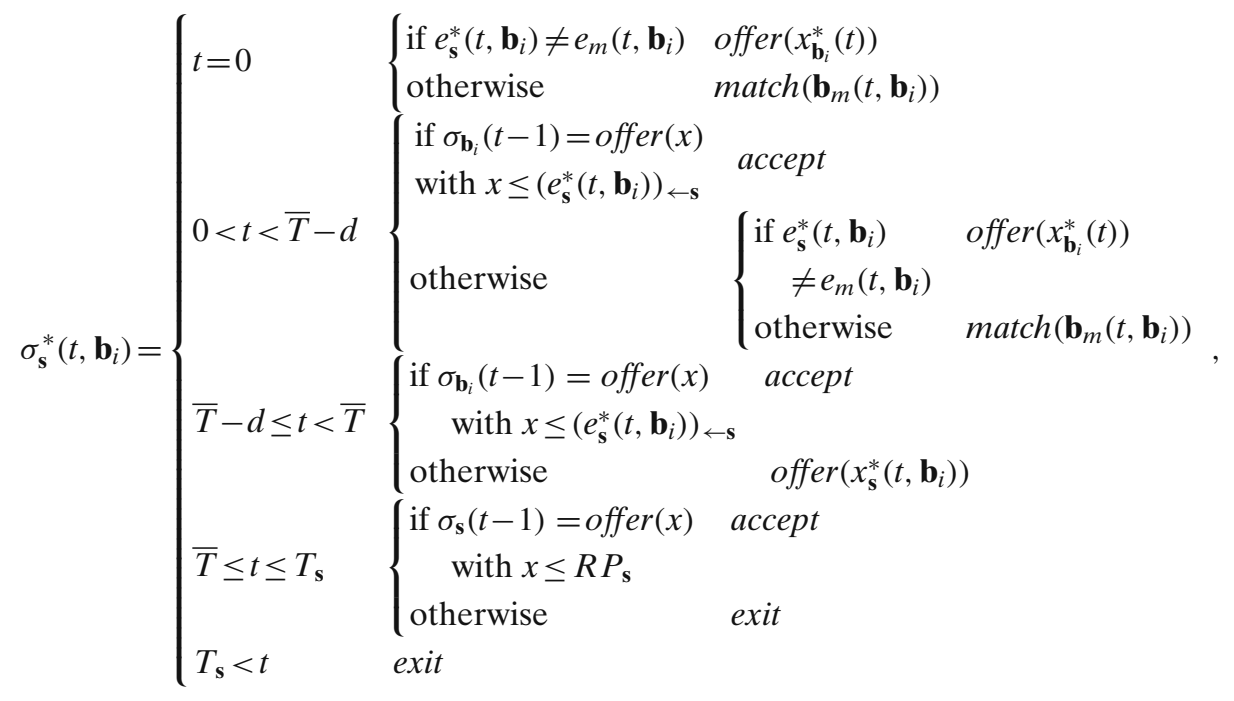


for all $i$ such that $\bar{T}-d \leq T_{\mathbf{b}_{i}}<\bar{T}$ and $R P_{\mathbf{b}_{i}}>R P_{\mathbf{s}}$, and for all time points $t \geq$ $\max \left\{A_{\mathbf{b}_{i}}, A_{\mathbf{s}}\right\}$ :

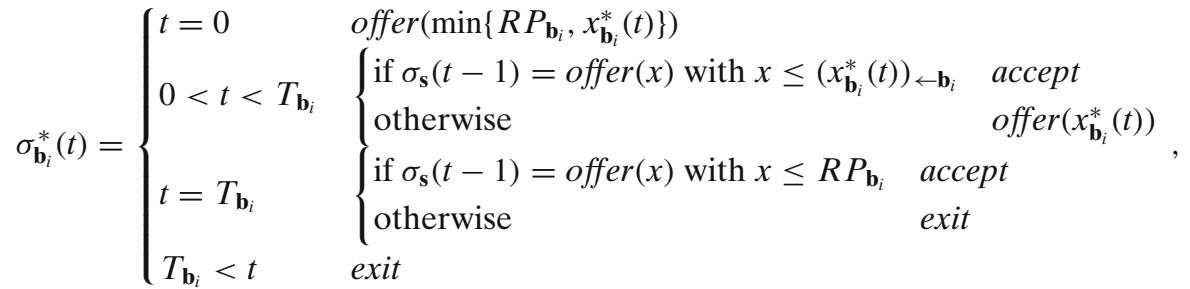

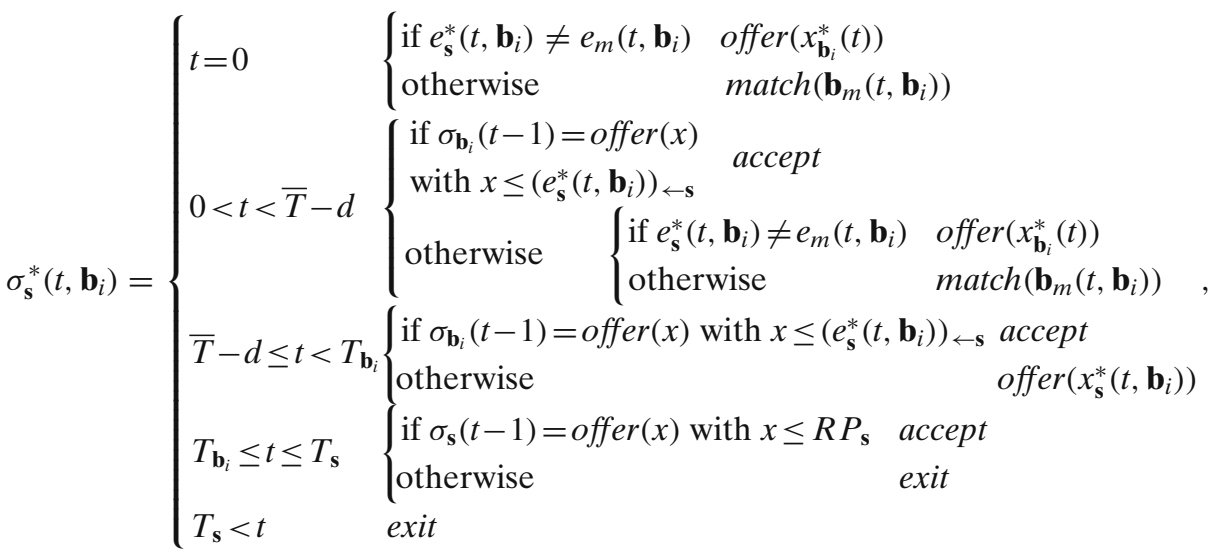

for all $i$ such that $T_{\mathbf{b}_{i}}<\bar{T}-d$ and $R P_{\mathbf{b}_{i}}>R P_{\mathbf{s}}$, and for all time points $t \geq$ $\max \left\{A_{\mathbf{b}_{i}}, A_{\mathbf{s}}\right\}$ :

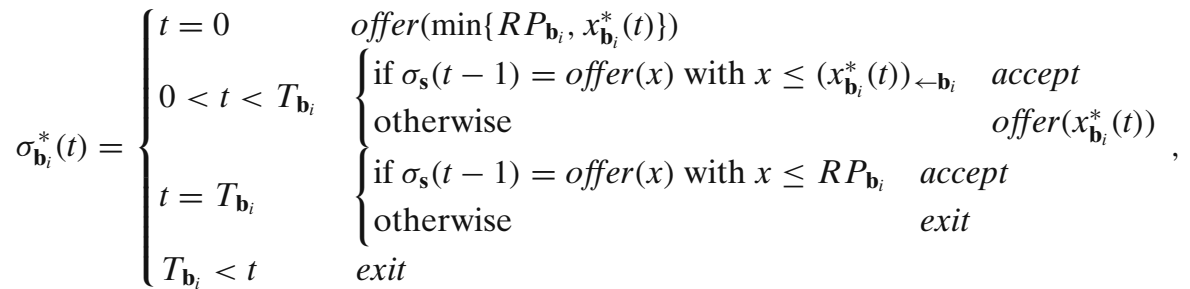

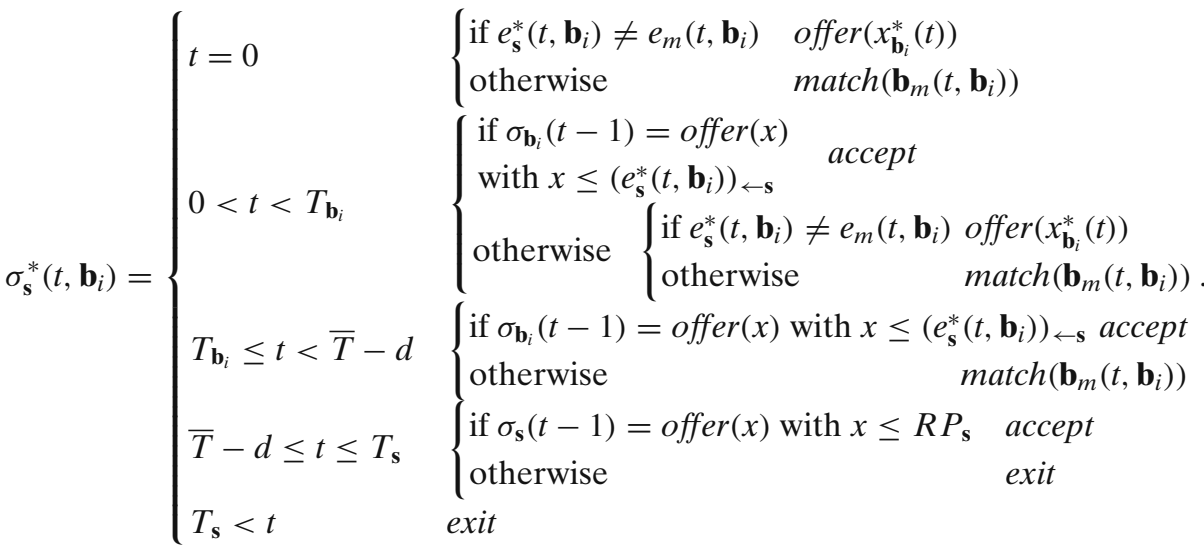


We state the following theorem (it holds by construction, being the above strategies produced by backward induction).

Theorem 5.6 The strategies provided in Table 4 constitute a subgame perfect equilibrium.

We report in Fig. 6 the backward induction construction in the presence of $B=$ $\left\{\mathbf{b}_{1}, \mathbf{b}_{2}, \mathbf{b}_{3}\right\}$ with $R P_{\mathbf{b}_{1}}=R P_{\mathbf{b}_{2}}=R P_{\mathbf{b}_{3}}=1, \delta_{\mathbf{b}_{1}}=\delta_{\mathbf{b}_{2}}=\delta_{\mathbf{b}_{3}}=0.95, T_{\mathbf{b}_{1}}=8, T_{\mathbf{b}_{2}}=13$, $T_{\mathbf{b}_{3}}=16, A_{\mathbf{b}_{1}}=-1, A_{\mathbf{b}_{2}}=-1, A_{\mathbf{b}_{3}}=-1, R P_{\mathbf{s}}=0, \delta_{\mathbf{s}}=0.95, T_{\mathbf{s}}=18, A_{\mathbf{s}}=-1$, and $d=1$. We report the optimal offers of the agents where they are defined and the optimal equivalent values where the optimal offer are not defined; we emphasize
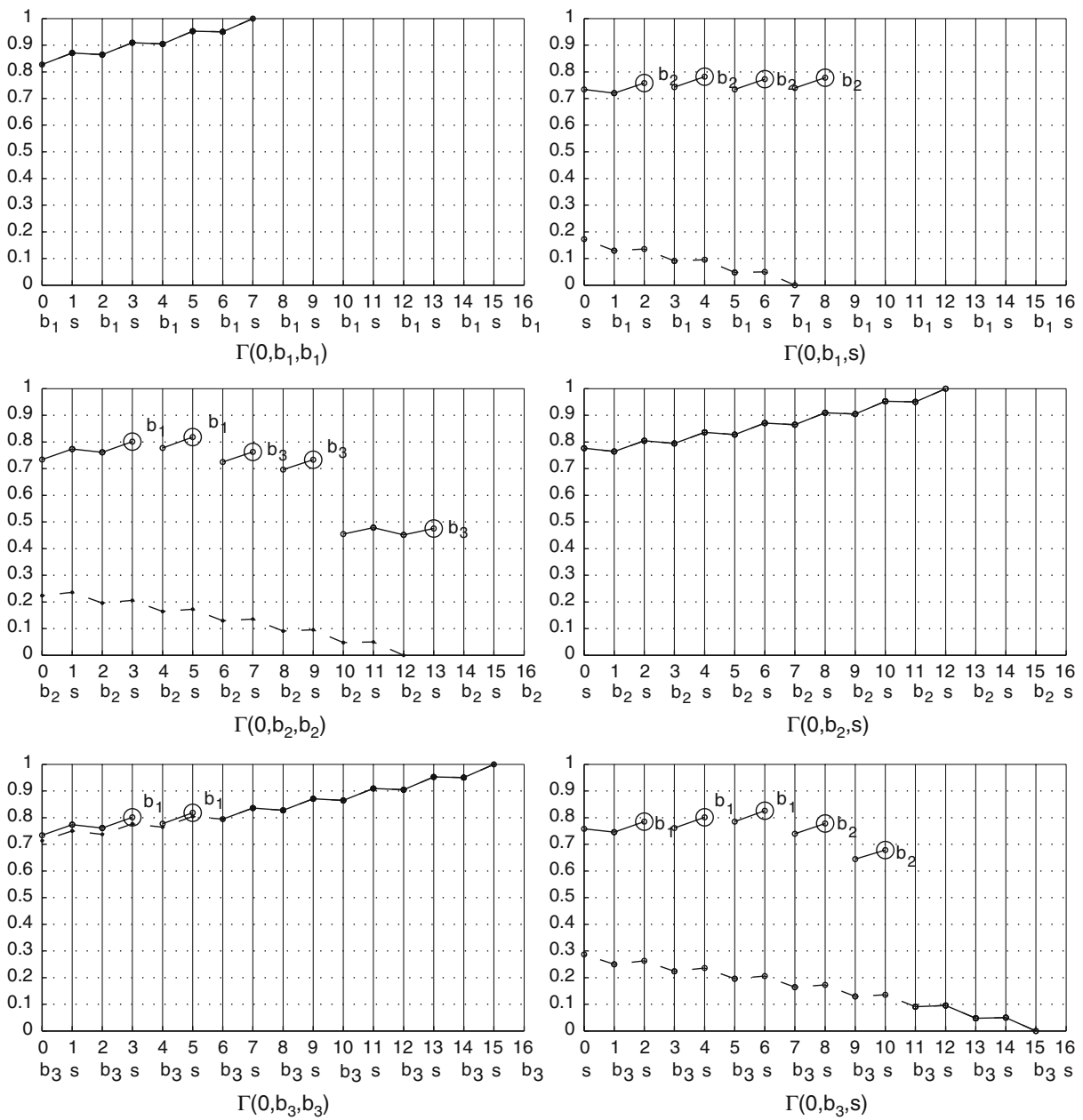

Fig. 6 Backward induction constructions with $R P_{\mathbf{b}_{1}}=R P_{\mathbf{b}_{2}}=R P_{\mathbf{b}_{3}}=1, \delta_{\mathbf{b}_{1}}=\delta_{\mathbf{b}_{2}}=\delta_{\mathbf{b}_{3}}=0.95$, $T_{\mathbf{b}_{1}}=8, T_{\mathbf{b}_{2}}=13, T_{\mathbf{b}_{3}}=16, A_{\mathbf{b}_{1}}=-1, A_{\mathbf{b}_{2}}=-1, A_{\mathbf{b}_{3}}=-1, R P_{\mathbf{s}}=0, \delta_{\mathbf{s}}=0.95, T_{\mathbf{s}}=18, A_{\mathbf{s}}=$ -1 , and $d=1$ 
the optimal equivalent values with a large circle and we specify the buyer to match aside the circle; the dashed line denotes the constructions when action match $(\cdot)$ is not available. The three buyers are present in the market from the initial time. On the equilibrium path $\mathbf{s}$ matches $\mathbf{b}_{1}$ at $t=-1$ and, independently of what agent opens the negotiation at $t=0$, the negotiation will close at $t=1$. (Indeed, if $\mathbf{s}$ opens the negotiation, she will make an offer that $\mathbf{b}_{1}$ will accept at the next time point.) It can be observed that the price at which agents draw up their contract in $\Gamma\left(0, \mathbf{b}_{1}, \mathbf{s}\right)$ raises with respect to the situation wherein $\mathbf{s}$ cannot exploit outside option. And therefore $\mathbf{s}$ expects to gain more.

We report in Fig. 7 the backward induction construction with the same setting considered in Fig. 6, but in this case $\mathbf{b}_{2}$ and $\mathbf{b}_{3}$ enter the market at $t=3$ and the buyers' reservation prices are different: $R P_{\mathbf{b}_{1}}=0.7, R P_{\mathbf{b}_{2}}=0.9, R P_{\mathbf{b}_{3}}=1$. Several
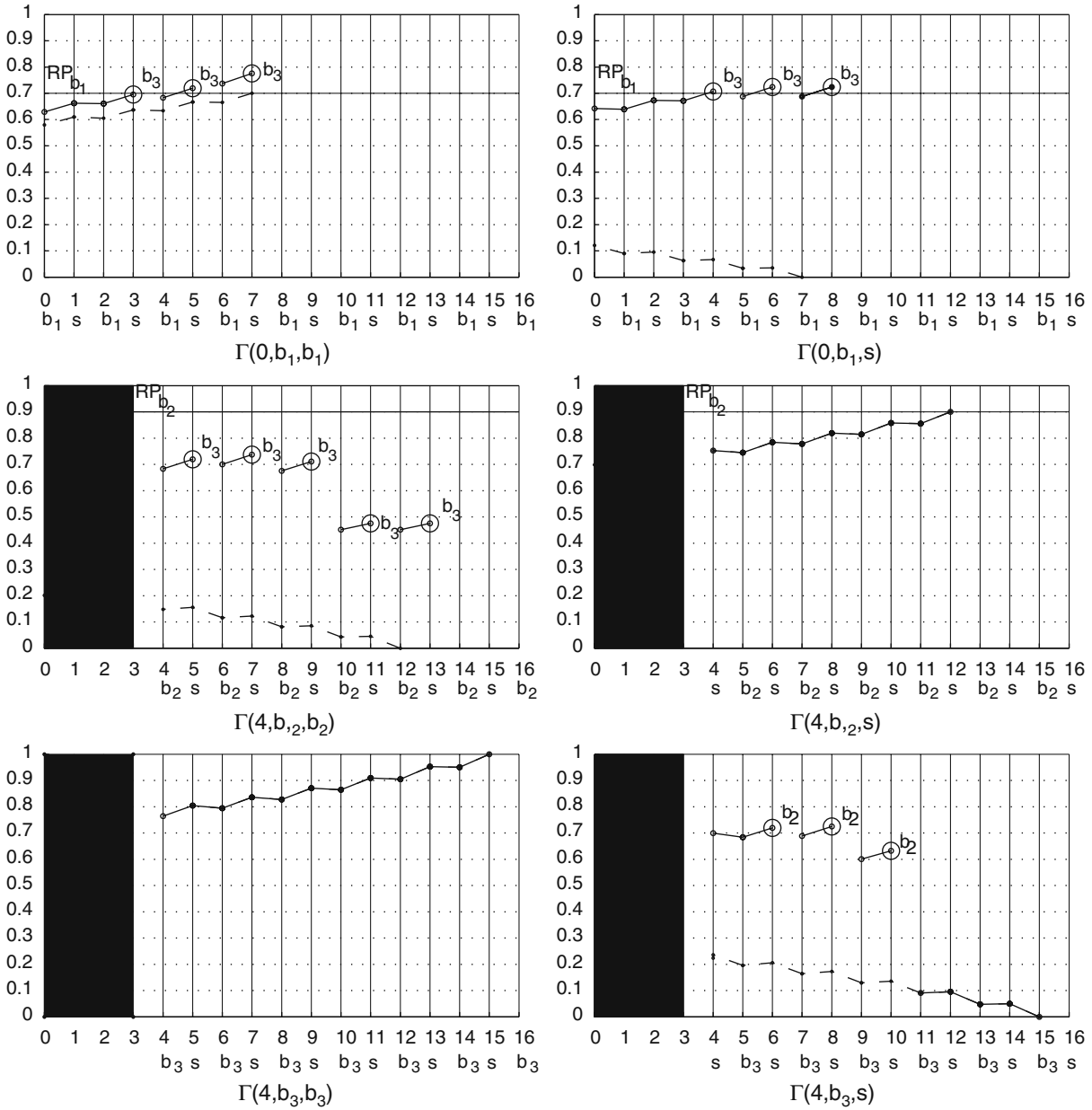

Fig. 7 Backward induction constructions with $R P_{\mathbf{b}_{1}}=0.7, R P_{\mathbf{b}_{2}}=0.9, R P_{\mathbf{b}_{3}}=1, \delta_{\mathbf{b}_{1}}=\delta_{\mathbf{b}_{2}}=\delta_{\mathbf{b}_{3}}=$ $0.95, T_{\mathbf{b}_{1}}=8, T_{\mathbf{b}_{2}}=13, T_{\mathbf{b}_{3}}=16, A_{\mathbf{b}_{1}}=-1, A_{\mathbf{b}_{2}}=3, A_{\mathbf{b}_{3}}=3, R P_{\mathbf{s}}=0, \delta_{\mathbf{s}}=0.95, T_{\mathbf{s}}=18, A_{\mathbf{s}}=$ -1 , and $d=1$ 
differences between Figs. 6 and 7 can be observed. Since in the setting depicted in Fig. 7 the buyers $\mathbf{b}_{2}$ and $\mathbf{b}_{3}$ enter the market at $t=3$, the sequences of the optimal offers and equivalent values begin at $t=4$. Indeed, $\mathbf{s}$ can match them only at $t=3$ and then the corresponding negotiations, being $d=1$, begin at $t=4$. Moreover, $\mathbf{b}_{1}$ makes an offer unacceptable for $\mathbf{s}$ at $t=6$ in $\Gamma\left(0, \mathbf{b}_{1}, \mathbf{b}_{1}\right)$. Indeed, it can be observed in figure that $x_{\mathbf{b}_{1}}^{*}(6)>R P_{\mathbf{b}_{1}}$. Also in this case $\mathbf{s}$ will match $\mathbf{b}_{1}$ at $t=-1$ on the equilibrium path, and the negotiation will close at $t=1$.

The computation of the strategies is essentially straightforward and requires exclusively the calculation of the sequences $x_{\mathbf{b}_{i}}^{*} \mathrm{~s}, x_{\mathbf{s}}^{*}\left(t, \mathbf{b}_{i}\right) \mathrm{s}, e_{\mathbf{s}}^{*}\left(t, \mathbf{b}_{i}\right) \mathrm{s}$ for every $i$. Once these values are found, they can be used in the strategies prescribed in Table 4. We report our solving algorithm in Algorithm 1. The computational complexity of the proposed algorithm is $O(m \cdot \bar{T})$. Notice that asymptotic computational complexity of solving the considered setting is the same one of solving the setting where $\mathbf{s}$ has not any outside option. Therefore, although we have enriched the negotiation model capturing competition among buyers, we have not raised the asymptotic computational complexity. In presence of multiple issues, the computational complexity is $O(l \cdot m \cdot \bar{T})$, where $l$ is the number of issues to negotiate.

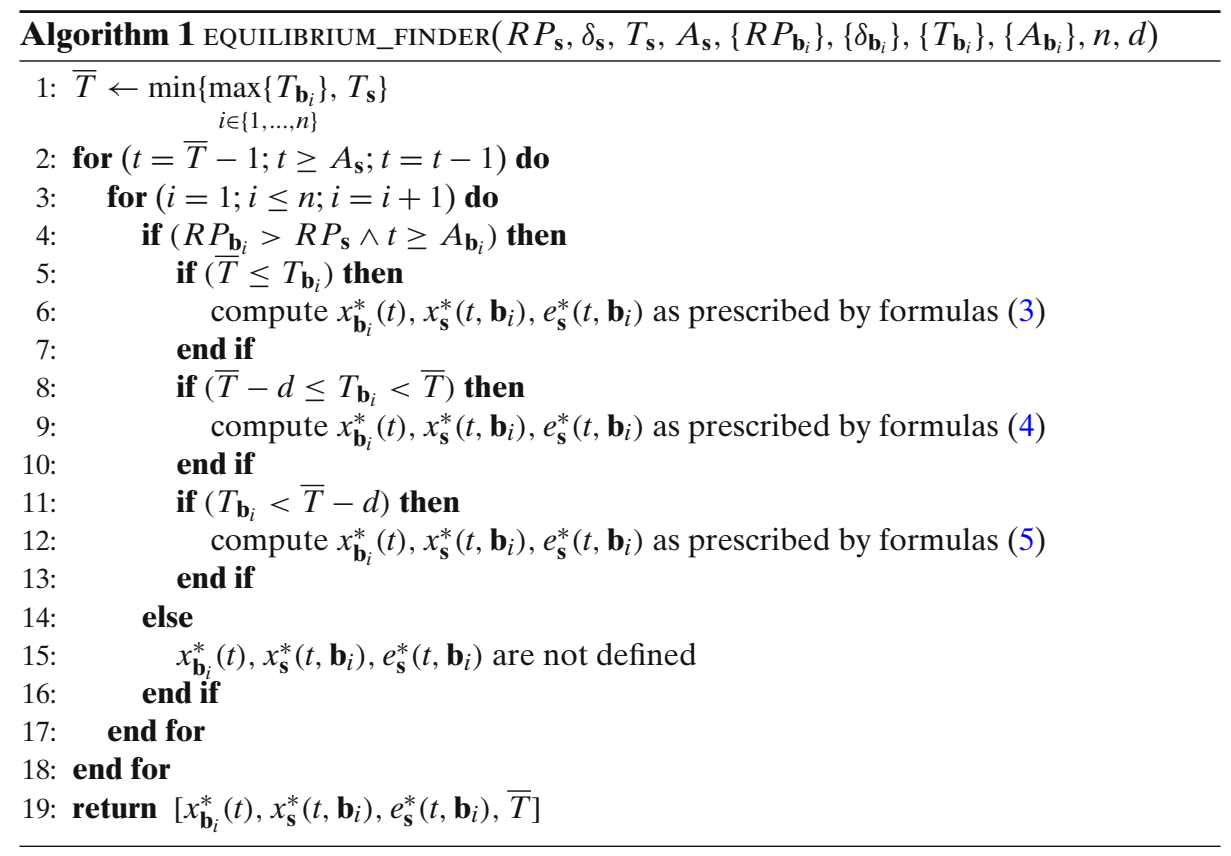

\section{B Proofs}

We discuss below the proofs of the main theoretical results provided along the paper.

\section{B.1 Buyer's optimal action with $\mathbf{b}_{1}, \mathbf{b}_{2}$}

This section is devoted to prove that the optimal action of $\mathbf{b}_{i}$ in $\Gamma\left(t, \mathbf{b}_{i}, \mathbf{b}_{i}\right)$ at $t$ is to offer $\left(e_{\mathbf{s}}^{*}\left(t+1, \mathbf{b}_{i}\right)\right)_{\leftarrow \mathbf{s}}$. We consider the situation where $d=1$. It can be easily shown 
that the theoretical results we provided below hold also when $d>1$. We initially state the following lemma.

Lemma 5.7 Given a bargaining situation $\Gamma\left(t, \mathbf{b}_{2}, \mathbf{b}_{2}\right)$ with $R P_{\mathbf{b}_{1}}=R P_{\mathbf{b}_{1}}=R P_{\mathbf{b}}$ and $A_{\mathbf{b}_{1}}=A_{\mathbf{b}_{2}}$ and such that on the equilibrium path:

- $\quad \mathbf{s}$ 's optimal actions from $t+1$ are to make two consecutive matches, i.e., $\sigma_{\mathbf{s}}^{*}(t+$ $\left.1, \mathbf{b}_{2}\right)=\operatorname{match}\left(\mathbf{b}_{1}\right)$ and $\sigma_{\mathbf{s}}^{*}\left(t+2, \mathbf{b}_{1}\right)=\operatorname{match}\left(\mathbf{b}_{2}\right)$,

- $\quad \mathbf{b}_{1}$ 's optimal action at $t+2$ is to make an offer that $\mathbf{s}$ will accept at the next time point,

- $\quad \mathbf{s}$ 's and $\mathbf{b}_{2}$ 's optimal actions at $t+3$ are to make offers that their opponent will accept,

then the optimal action of $\mathbf{b}_{2}$ at $t$ is to offer $\left(e_{\mathbf{s}}^{*}\left(t+1, \mathbf{b}_{2}\right)\right)_{\leftarrow \mathbf{s}}$.

Proof Trivially, among all the offers that $\mathbf{s}$ will accept at $t+1,\left(e_{\mathbf{s}}^{*}\left(t+1, \mathbf{b}_{2}\right)\right)_{\leftarrow \mathbf{s}}$ is the offer that maximizes the utility of $\mathbf{b}_{2}$. We must show that $\mathbf{b}_{2}$ does not prefer to make an offer that is unacceptable for $\mathbf{s}$ rather than to offer $\left(e_{\mathbf{s}}^{*}\left(t+1, \mathbf{b}_{2}\right)\right)_{\leftarrow \mathbf{s}}$. We calculate the equivalent value for $\mathbf{b}_{2}$ to make at $t$ an offer unacceptable for $\mathbf{s}$ at $t+1$ and we compare it with $\left(e_{\mathbf{s}}^{*}\left(t+1, \mathbf{b}_{2}\right)\right)_{\leftarrow \mathbf{s}}$. We denote by $e_{\mathbf{b}_{2}}$ (unacc.) the equivalent value for $\mathbf{b}_{2}$ to make at $t$ an offer unacceptable for $\mathbf{s}$ at $t+1$. We denote by $\bar{x}$ the average between the offer that would make $\mathbf{b}_{2}$ at $t+3$ in $\Gamma\left(t+3, \mathbf{b}_{2}, \mathbf{b}_{2}\right)$ and the offer that would make $\mathbf{s}$ at $t+3$ in $\Gamma\left(t+3, \mathbf{b}_{2}, \mathbf{s}\right)$ : it is $\bar{x}=\frac{1}{2} x_{\mathbf{b}_{2}}^{*}(t+3)+$ $\frac{1}{2} x_{\mathbf{s}}^{*}\left(t+3, \mathbf{b}_{2}\right)$. The equivalent value $e_{\mathbf{b}_{2}}$ (unacc.) can be expressed in function of $\bar{x}$ as follows: $\bar{x}=\left(\frac{1}{2} \bar{x}_{\leftarrow \mathbf{b}_{2}}+\frac{1}{2} R P_{\mathbf{b}}\right)_{\leftarrow 2\left[\mathbf{b}_{2}\right]}$. Indeed, $\mathbf{b}_{2}$ expects a utility of zero at $t+2$ with a probability of 0.5 and $\bar{x}$ at $t+3$ with a probability of 0.5 . We can provide an upper bound of $\left(e_{\mathbf{s}}^{*}\left(t+1, \mathbf{b}_{2}\right)\right)_{\leftarrow \mathbf{s}}$ in function of $\bar{x}$. Precisely, it is $\left(e_{\mathbf{s}}^{*}\left(t+1, \mathbf{b}_{2}\right)\right)_{\leftarrow \mathbf{s}}<$ $\left(\frac{1}{2} \bar{x}_{\leftarrow \mathbf{s}}+\frac{1}{2} R P_{\mathbf{b}}\right)_{\leftarrow 2[\mathbf{s}]}$. Indeed, the exact value of $\left(e_{\mathbf{s}}^{*}\left(t+1, \mathbf{b}_{2}\right)\right)_{\leftarrow \mathbf{s}}$ is $\left(\frac{1}{2} \bar{x}_{\leftarrow \mathbf{s}}+\frac{1}{2} x_{\mathbf{b}_{1}}^{*}(t+\right.$ $2))_{\leftarrow 2[\mathbf{s}]}$ and the value of $x_{\mathbf{b}_{1}}^{*}(t+2)$ whereby $\left(e_{\mathbf{s}}^{*}\left(t+1, \mathbf{b}_{2}\right)\right)_{\leftarrow \mathbf{s}}$ is maximum is $R P_{\mathbf{b}}$. It can be easily observed that $\left(e_{\mathbf{s}}^{*}\left(t+1, \mathbf{b}_{2}\right)\right)_{\leftarrow \mathbf{s}}<\left(\frac{1}{2} \bar{x}_{\leftarrow \mathbf{s}}+\frac{1}{2} R P_{\mathbf{b}}\right)_{\leftarrow 2[\mathbf{s}]}<\left(\frac{1}{2} \bar{x}_{\leftarrow \mathbf{b}_{2}}+\right.$ $\left.\frac{1}{2} R P_{\mathbf{b}}\right)_{\leftarrow 2\left[\mathbf{b}_{2}\right]}=e_{\mathbf{b}_{2}}$ (unacc.). Therefore, $\mathbf{b}_{2}$ prefers to offer $\left(e_{\mathbf{s}}^{*}\left(t+1, \mathbf{b}_{2}\right)\right)_{\leftarrow \mathbf{s}}$ rather than to make any other action.

Now we generalize Lemma 5.7 as follows.

Lemma 5.8 Given a bargaining situation $\Gamma\left(t, \mathbf{b}_{2}, \mathbf{b}_{2}\right)$ with $R P_{\mathbf{b}_{1}}=R P_{\mathbf{b}_{1}}=R P_{\mathbf{b}}$ and $A_{\mathbf{b}_{1}}=A_{\mathbf{b}_{2}}$ and such that on the equilibrium path:

- $\mathbf{s}$ 's optimal actions from $t+1$ is to make $k>1$ consecutive matches with $k$ even, i.e., $\sigma_{\mathbf{s}}^{*}\left(t+1, \mathbf{b}_{2}\right)=\operatorname{match}\left(\mathbf{b}_{1}\right), \sigma_{\mathbf{s}}^{*}\left(t+2, \mathbf{b}_{1}\right)=\operatorname{match}\left(\mathbf{b}_{2}\right), \sigma_{\mathbf{s}}^{*}\left(t+3, \mathbf{b}_{2}\right)=$ $\operatorname{match}\left(\mathbf{b}_{1}\right)$, and so on up to $\sigma_{\mathbf{s}}^{*}\left(t+k, \mathbf{b}_{1}\right)=\operatorname{match}\left(\mathbf{b}_{2}\right)$,

- $\mathbf{b}_{1}$ 's optimal action at $t+2 l$ with $l \in\left\{1, \ldots, \frac{k}{2}\right\}$ is to make an offer that $\mathbf{s}$ will accept at the next time point,

- $\mathbf{b}_{2}$ 's optimal action at $t+2 l+1$ with $l \in\left\{1, \ldots, \frac{k}{2}-1\right\}$ is to make an offer that $\mathbf{s}$ will accept at the next time point,

- $\quad \mathbf{s}$ 's and $\mathbf{b}_{2}$ 's optimal actions at $t+k+1$ are to make offers that their opponent will accept,

then the optimal action of $\mathbf{b}_{2}$ at $t$ is to offer $\left(e_{\mathbf{s}}^{*}\left(t+1, \mathbf{b}_{2}\right)\right)_{\leftarrow \mathbf{s}}$. 
Proof sketch Similarly to analysis provided in the proof of Lemma 5.7, we can calculate the equivalent value for $\mathbf{b}_{2}$ of making at $t$ an offer unacceptable for $\mathbf{s}$ at the next time point and we can compare it with respect to $\left(e_{\mathbf{s}}^{*}\left(t+1, \mathbf{b}_{2}\right)\right)_{\leftarrow \mathbf{s}}$. We denote by $\bar{x}$ the average between the offer that would make $\mathbf{b}_{2}$ at $t+k+1$ in $\Gamma(t+$ $\left.k+1, \mathbf{b}_{2}, \mathbf{b}_{2}\right)$ and the offer that would make $\mathbf{s}$ at $t+k+1$ in $\Gamma\left(t+k+1, \mathbf{b}_{2}, \mathbf{s}\right)$ : it is $\bar{x}=\frac{1}{2} x_{\mathbf{b}_{2}}^{*}(t+k+1)+\frac{1}{2} x_{\mathbf{s}}^{*}\left(t+k+1, \mathbf{b}_{2}\right)$. We can provide a lower bound of the equivalent value $e_{\mathbf{b}_{2}}$ (unacc.) in function of $\bar{x}$ as follows: $e_{\mathbf{b}_{2}}$ (unacc.) $>\left(R P_{\mathbf{b}} \sum_{i=1}^{k / 2}\left[2^{1-2 i}\right]+\right.$ $\left.\sum_{i=1}^{k / 2-1}\left[2^{-2 i} \cdot x_{\mathbf{b}_{2}}^{*}(t+2 i+1)\right]+\bar{x}_{\leftarrow \mathbf{b}_{2}}\right)_{\leftarrow 2\left[\mathbf{b}_{2}\right]}$. Similarly, we can provide an upper bound of $\left(e_{\mathbf{s}}^{*}\left(t+1, \mathbf{b}_{2}\right)\right)_{\leftarrow \mathbf{s}}$ in function of $\bar{x}$ as follows: $\left(e_{\mathbf{s}}^{*}\left(t+1, \mathbf{b}_{2}\right)\right)_{\leftarrow \mathbf{s}}<\left(R P_{\mathbf{b}} \sum_{i=1}^{k / 2}\left[2^{1-2 i}\right]+\right.$ $\left.\sum_{i=1}^{k / 2-1}\left[2^{-2 i} \cdot x_{\mathbf{b}_{2}}^{*}(t+2 i+1)\right]+\bar{x}_{\leftarrow \mathbf{s}}\right)_{\leftarrow 2[\mathbf{s}]}$. It can be easily observed that $\left(e_{\mathbf{s}}^{*}(t+\right.$ $\left.\left.1, \mathbf{b}_{2}\right)\right)_{\leftarrow \mathbf{s}}<e_{\mathbf{b}_{2}}$ (unacc.) and therefore $\mathbf{b}_{2}$ prefers to offer $\left(e_{\mathbf{s}}^{*}\left(t+1, \mathbf{b}_{2}\right)\right)_{\leftarrow \mathbf{s}}$ rather than to make any other action.

Analogously to what we made above, we can state the following lemma, whose proof is omitted since it is similar to the proof of Lemma 5.8.

Lemma 5.9 Given a bargaining situation $\Gamma\left(t, \mathbf{b}_{2}, \mathbf{b}_{2}\right)$ with $R P_{\mathbf{b}_{1}}=R P_{\mathbf{b}_{1}}=R P_{\mathbf{b}}$ and $A_{\mathbf{b}_{1}}=A_{\mathbf{b}_{2}}$ and such that on the equilibrium path:

- $\quad$ s's optimal actions from $t+1$ is to make $k>1$ consecutive matches with $k$ odd, i.e., $\sigma_{\mathbf{s}}^{*}\left(t+1, \mathbf{b}_{2}\right)=\operatorname{match}\left(\mathbf{b}_{1}\right), \sigma_{\mathbf{s}}^{*}\left(t+2, \mathbf{b}_{1}\right)=\operatorname{match}\left(\mathbf{b}_{2}\right), \sigma_{\mathbf{s}}^{*}\left(t+3, \mathbf{b}_{2}\right)=\operatorname{match}\left(\mathbf{b}_{1}\right)$, and so on up to $\sigma_{\mathbf{s}}^{*}\left(t+k, \mathbf{b}_{2}\right)=\operatorname{match}\left(\mathbf{b}_{1}\right)$,

- $\quad \mathbf{b}_{1}$ 's optimal action at $t+2 l+1$ with $l \in\left\{1, \ldots, \frac{k-1}{2}\right\}$ is to make an offer that $\mathbf{s}$ will accept at the next time point,

- $\quad \mathbf{b}_{2}$ 's optimal action at $t+2 l$ with $l \in\left\{1, \ldots, \frac{k-1}{2}-1\right\}$ is to make an offer that $\mathbf{s}$ will accept at the next time point,

- $\quad \mathbf{s}$ 's and $\mathbf{b}_{1}$ 's optimal actions at $t+k+1$ are to make offers that their opponent will accept,

then the optimal action of $\mathbf{b}_{2}$ at $t$ is to offer $\left(e_{\mathbf{s}}^{*}\left(t+1, \mathbf{b}_{2}\right)\right)_{\leftarrow \mathbf{s}}$.

On the basis of Lemmas 5.8 and 5.9 we can prove the following proposition.

Proposition 5.3 When the information is complete, the optimal action of $\mathbf{b}_{i}$ at $t$ is to offer $\left(e_{\mathbf{s}}^{*}\left(t+1, \mathbf{b}_{i}\right)\right)_{\leftarrow \mathbf{s}}$.

Proof In the following situations the proof is trivial:

1. when the optimal action of $\mathbf{s}$ at $t+1$ is to make an offer, i.e., when $e_{\mathbf{s}}^{*}\left(t+1, \mathbf{b}_{i}\right)=$ $\left(x_{\mathbf{b}_{i}}^{*}(t+2)\right)_{\leftarrow \mathbf{b}_{i}}$,

2. when the optimal action of $\mathbf{s}$ at $t+1$ is to make $\operatorname{match}\left(\mathbf{b}_{i}\right)$ and the equilibrium strategies in the subgames starting at $t+2$ where $\mathbf{s}$ negotiates with $\mathbf{b}_{i}$ prescribe that $\mathbf{s}$ and $\mathbf{b}_{i}$ reach an agreement at $t+3$.

Easily, in the first situation the analysis is exactly as in absence of buyers' competition and in the second situation $\mathbf{b}_{i}$ expects a utility of zero from offering an offer unacceptable for $\mathbf{s}$. The proof in the general situation can be produced by iteratively applying backward Lemmas 5.8 and 5.9 from $t=T_{\mathbf{b}_{1}}-1$. 
B.2 Conditions such that a buyer expects negative utility from starting a negotiation

We initially state the following lemma, whose proof is similar to the proof of Lemma B4 and then omitted.

Lemma 5.10 $A$ buyer $\mathbf{b}_{i}$ expects negative utility from starting a negotiation at $t$ when $x_{\mathbf{b}_{i}}^{*}(t)=R P_{\mathbf{b}_{i}}$ and $e_{\mathbf{s}}^{*}\left(t, \mathbf{b}_{i}\right)>R P_{\mathbf{b}_{i}}$.

We can relax the condition expressed in Lemma 5.10 on the basis of the following lemma.

Lemma 5.11 At any time point $t$, if $x_{\mathbf{b}_{i}}^{*}(t)=R P_{\mathbf{b}_{i}}$, then $e_{\mathbf{s}}^{*}\left(t, \mathbf{b}_{i}\right)>R P_{\mathbf{b}_{i}}$.

Proof We consider the special case where there are two buyers, $\mathbf{b}_{1}$ and $\mathbf{b}_{2}$, with $T_{\mathbf{b}_{1}}<$ $T_{\mathbf{b}_{2}}$. In what follows we prove the lemma in this special case. The proof in the general case can be provided by iterating the special case.

If $x_{\mathbf{b}_{i}}^{*}(t)=R P_{\mathbf{b}_{1}}$, then the optimal action of $\mathbf{s}$ at $t+1$ in $\Gamma\left(t+1, \mathbf{b}_{1}, \mathbf{s}\right)$ is $\operatorname{match}\left(\mathbf{b}_{2}\right)$. Therefore, the value of $\left(e_{\mathbf{s}}^{*}\left(t+1, \mathbf{b}_{1}\right)\right)_{\leftarrow \mathbf{s}}$ is:

$$
\left(e_{\mathbf{s}}^{*}\left(t+1, \mathbf{b}_{1}\right)\right)_{\leftarrow \mathbf{s}}=\left(\frac{1}{2} x_{\mathbf{b}_{2}}^{*}(t+d+1)+\frac{1}{2} e_{\mathbf{s}}^{*}\left(t+d+1, \mathbf{b}_{2}\right)\right)_{\leftarrow(d+1)[\mathbf{s}]},
$$

and, since $x_{\mathbf{b}_{1}}^{*}(t)=\min \left\{R P_{\mathbf{b}_{1}},\left(e_{\mathbf{s}}^{*}\left(t+1, \mathbf{b}_{1}\right)\right)_{\leftarrow \mathbf{s}}\right\}$, we have $\left(e_{\mathbf{s}}^{*}\left(t+1, \mathbf{b}_{1}\right)\right)_{\leftarrow \mathbf{s}} \geq x_{\mathbf{b}_{1}}^{*}(t)$. Since by definition $e_{\mathbf{s}}^{*}\left(t, \mathbf{b}_{1}\right) \geq e_{m}\left(t, \mathbf{b}_{1}\right)$, in order to prove that if $x_{\mathbf{b}_{1}}^{*}(t) \geq R P_{\mathbf{b}_{1}}$ then $e_{\mathbf{s}}^{*}\left(t, \mathbf{b}_{1}\right)>R P_{\mathbf{b}_{1}}$, we can prove that if $\left(e_{\mathbf{s}}^{*}\left(t+1, \mathbf{b}_{1}\right)\right)_{\leftarrow \mathbf{s}} \geq R P_{\mathbf{b}_{1}}$ then $e_{m}\left(t, \mathbf{b}_{1}\right)>R P_{\mathbf{b}_{1}}$. The value of $e_{m}\left(t, \mathbf{b}_{1}\right)$ is:

$$
e_{m}\left(t, \mathbf{b}_{1}\right)=\left(\frac{1}{2} x_{\mathbf{b}_{2}}^{*}(t+d)+\frac{1}{2} e_{\mathbf{s}}^{*}\left(t+d, \mathbf{b}_{2}\right)\right)_{\leftarrow d[\mathbf{s}]},
$$

where $x_{\mathbf{b}_{2}}^{*}(t+d)=\left(e_{\mathbf{s}}^{*}\left(t+d+1, \mathbf{b}_{2}\right)\right)_{\leftarrow \mathbf{s}}$ and $e_{\mathbf{s}}^{*}\left(t+d, \mathbf{b}_{2}\right) \geq\left(x_{\mathbf{b}_{2}}^{*}(t+d+1)\right)_{\leftarrow \mathbf{b}_{2}}$. By expressing the value of $e_{m}\left(t, \mathbf{b}_{1}\right)-\left(e_{\mathbf{s}}^{*}\left(t+1, \mathbf{b}_{1}\right)\right)_{\leftarrow \mathbf{s}}$ we obtain:

$$
e_{m}\left(t, \mathbf{b}_{1}\right)-\left(e_{\mathbf{s}}^{*}\left(t+1, \mathbf{b}_{1}\right)\right)_{\leftarrow \mathbf{s}} \geq\left(x_{\mathbf{b}_{2}}^{*}(t+d+1)\right)_{\leftarrow \mathbf{b}_{2}}-x_{\mathbf{b}_{2}}^{*}(t+d+1) \delta_{\mathbf{s}}+3 R P_{\mathbf{s}}\left(1-\delta_{\mathbf{s}}\right)>0 .
$$

Hence, we obtain the thesis.

Finally, we combine Lemmas 5.10 and 5.11 as follows.

Proposition 5.4 A buyer $\mathbf{b}_{i}$ expects negative utility from starting a negotiation at $t$ when $x_{\mathbf{b}_{i}}^{*}(t)=R P_{\mathbf{b}_{i}}$.

\section{References}

1. Binmore, K., Shaked, A., Sutton, J.: An outside option expirement. Q. J. Econ. 104(4), 753-770 (1989)

2. Ceppi, S., Gatti, N.: An algorithmic game theory framework for solving bargaining with uncertainty. Tech. Rep. 2009.26, Politecnico di Milano, Dipartimento di Elettronica e Informazione (2009) 
3. Chatterjee, K., Samuelson, L.: Bargaining under two-sided incomplete information: the unrestricted offers case. Oper. Res. 36(4), 605-618 (1988)

4. Cramton, P.C., Ausubel, L.M., Deneckere, R.J.: Handbook of Game Theory, vol. 3, pp. 18971945. Elsevier, Amsterdam (2002)

5. Dagan, N., Serrano, R., Volij, O.: Bargaining, coalitions and competition. Econ. Theory 15(2), 279-296 (2000)

6. Di Giunta, F., Gatti, N.: Alternating-offers bargaining under one-sided uncertainty on deadlines. In: Proceedings of the European Conference on Artificial Intelligence (ECAI), pp. 225-229. Riva del Garda, Italy (2006)

7. Di Giunta, F., Gatti, N.: Bargaining in-bundle over multiple issues in finite-horizon alternatingoffers protocol. In: Proceedings of the Symposium on Artificial Intelligence and Mathematics (AIMATH). Fort Lauderdale, USA (2006)

8. Di Giunta, F., Gatti, N.: Bargaining over multiple issues in finite horizon alternating-offers protocol. Ann. Math. Artif. Intell. 47(3-4), 251-271 (2006)

9. Fatima, S.S., Wooldridge, M., Jennings, N.R.: An agenda-based framework for multi-issue negotiation. Artif. Intell. 152(1), 1-45 (2004)

10. Fatima, S.S., Wooldridge, M., Jennings, N.R.: On efficient procedures for multi-issue negotiation. In: Proceedings of Trading Agent Design and Analysis and Agent Mediated Electronic Commerce (TADA-AMEC) at the International Joint Conference on Autonomous Agents and Multi-Agent Systems (AAMAS), pp. 71-84. Hakodate, Japan (2006)

11. Fatima, S.S., Wooldridge, M.J., Jennings, N.R.: Multi-issue negotiation with deadlines. J. Artif. Intell. Res. 27(1), 381-417 (2006)

12. Fudenberg, D., Levine, D.K., Tirole, J.: Incomplete information bargaining with outside opportunities. Q. J. Econ. 102(1), 37-50 (1987)

13. Fudenberg, D., Tirole, J.: Sequential bargaining with incomplete information. Rev. Econ. Stud. 50(2), 221-247 (1983)

14. Fudenberg, D., Tirole, J.: Game Theory. The MIT Press, Cambridge, USA (1991)

15. Gale, D.: Bargaining and competition. part I: Characterization. Econometrica 54(4), 785-806 (1986)

16. Gale, D.: Bargaining and competition. part II: existence. Econometrica 54(4), 807-818 (1986)

17. Gale, D.: Strategic Foundations of General Equilibrium-Dynamic Matching and Bargaining Games. Cambridge University Press, Cambridge (2000)

18. Gale, D., Shapley, L.S.: College admissions and the stability of marriage. Am. Math. Mon. 69(1), 9-14 (1962)

19. Gatti, N.: Bargaining in markets with one-sided competition: model and analysis. In: Proceedings of the ACM/IEEE International Conference on Agent Intelligent Technologies (IAT), vol. IAT, pp. 443-446. Sydney, Australia (2008)

20. Gatti, N., Di Giunta, F., Marino, S.: Alternating-offers bargaining with one-sided uncertain deadlines: an efficient algorithm. Artif. Intell. 172(8-9), 1119-1157 (2008)

21. Gatti, N., Lazaric, A., Restelli, M.: Towards automated bargaining in electronic markets: a partially two-sided competition model. In: Proceedings of the Workshop on Agent-Mediated Electronic Commerce (AMEC) in the International Joint Conference on Autonomous Agents and Multi-Agent Systems (AAMAS), pp. 29-42. Lisbon, Portugal (2008)

22. Gilpin, A., Sandholm, T.: Finding equilibria in large sequential games of imperfect information. In: Proceedings of the ACM Conference on Electronic Commerce (EC), pp. 160-169. ACM, New York (2006)

23. Halmos, P.R.: Measure Theory. Springer, Berlin (1974)

24. Harsanyi, J.C., Selten, R.: A generalized Nash solution for two-person bargaining games with incomplete information. Manage. Sci. 18, 80-106 (1972)

25. Jehiel, P., Moldovanu, P.: Cyclical delay in bargaining with externalities. Rev. Econ. Stud. 62(4), 619-637 (1995)

26. Jennings, N.R., Faratin, P., Lomuscio, A.R., Parsons, S., Sierra, C., Wooldridge, M.: Automated negotiation: prospects, methods, and challenges. International Journal of Group Decision and Negotiation 10(2), 199-215 (2001)

27. Koller, D., Megiddo, N., von Stengel, B.: Fast algorithms for finding randomized strategies in game trees. In: Proceedings of the Annual ACM Symposium on Theory of Computing (STOC), pp. 750-759. ACM, New York (1994)

28. Koller, D., Pfeffer, A.: Representations and solutions for game-theoretic problem. Artif. Intell. 94(1), 167-215 (1997)

29. Kraus, S.: Strategic Negotiation in Multiagent Environments. MIT, Cambridge (2001) 
30. Kreps, D.R., Wilson, R.: Sequential equilibria. Econometrica 50(4), 863-894 (1982)

31. Lazaric, A., Munoz de Cote, E., Gatti, N., Restelli, M.: Reinforcement learning in extensive form games with incomplete information: the bargaining case study. In: Proceedings of the International Joint Conference on Autonomous Agents and Multi-Agent Systems (AAMAS), pp. 216-218. Honolulu, USA (2007)

32. Martello, S., Toth, P.: Knapsack Problems: Algorithms and Computer Implementations. Wiley, New York (1990)

33. Miltersen, P.B., Sorensen, T.B.: Computing sequential equilibria for two-player games. In: Proceedings of the ACM-SIAM Symposium on Discrete Algorithm, pp. 107-116. ACM, New York (2006)

34. Napel, S.: Bilateral Bargaining: Theory and Applications. Springer, Berlin (2002)

35. Nguyen, T.D., Jennings, N.R.: Coordinating multiple concurrent negotiations. In: Proceedings of the International Joint Conference on Autonomous Agents and Multi-Agent Systems (AAMAS), pp. 1064-1071. New York, USA (2004)

36. Osborne, M.J., Rubinstein, A.: Bargaining and Markets. Academic, San Diego (1990)

37. Rosenschein, J.S., Zlotkin, G.: Rules of Encounter. Designing Conventions for Automated Negotiations among Computers. MIT, Cambridge (1994)

38. Rubinstein, A.: Perfect equilibrium in a bargaining model. Econometrica 50(1), 97-109 (1982)

39. Rubinstein, A.: A bargaining model with incomplete information about time preferences. Econometrica 53(5), 1151-1172 (1985)

40. Rubinstein, A., Wolinsky, A.: Equilibrium in a market with sequential bargaining. Econometrica 53(3), 1133-1150 (1985)

41. Rubinstein, A., Wolinsky, A.: Decentralized trading, strategic behavior and the walrasian outcome. Rev. Econ. Stud. 56(1), 63-78 (1990)

42. Sandholm, T.: Agents in electronic commerce: component technologies for automated negotiation and coalition formation. Autonomous Agents and Multi-Agent Systems 3(1), 73-96 (2000)

43. Sandholm, T., Vulkan, N.: Bargaining with deadlines. In: Proceedings of the American Association for Artificial Intelligence Conference (AAAI), pp. 44-51. Orlando, USA (1999)

44. Serrano, R.: The New Palgrave: a Dictionary of Economics, 2nd edn., chap. Bargaining. McMillian, London (2008)

45. Ståhl, I.: Bargaining Theory. Stockholm School of Economics, Stockholm (1972) 